\title{
Heart failure self-management : balancing between medical and psychosocial needs : evaluation of a cognitive-behavioural self-management group programme
}

Citation for published version (APA):

Smeulders, E. S. T. F. (2010). Heart failure self-management : balancing between medical and psychosocial needs : evaluation of a cognitive-behavioural self-management group programme. [Doctoral Thesis, Maastricht University]. Maastricht University. https://doi.org/10.26481/dis.20100226ess

Document status and date:

Published: 01/01/2010

DOI:

10.26481/dis.20100226ess

Document Version:

Publisher's PDF, also known as Version of record

Please check the document version of this publication:

- A submitted manuscript is the version of the article upon submission and before peer-review. There can be important differences between the submitted version and the official published version of record. People interested in the research are advised to contact the author for the final version of the publication, or visit the DOI to the publisher's website.

- The final author version and the galley proof are versions of the publication after peer review.

- The final published version features the final layout of the paper including the volume, issue and page numbers.

Link to publication

\footnotetext{
General rights rights.

- You may freely distribute the URL identifying the publication in the public portal. please follow below link for the End User Agreement:

www.umlib.nl/taverne-license

Take down policy

If you believe that this document breaches copyright please contact us at:

repository@maastrichtuniversity.nl

providing details and we will investigate your claim.
}

Copyright and moral rights for the publications made accessible in the public portal are retained by the authors and/or other copyright owners and it is a condition of accessing publications that users recognise and abide by the legal requirements associated with these

- Users may download and print one copy of any publication from the public portal for the purpose of private study or research.

- You may not further distribute the material or use it for any profit-making activity or commercial gain

If the publication is distributed under the terms of Article 25fa of the Dutch Copyright Act, indicated by the "Taverne" license above, 
Heart failure self-management: balancing

between medical and psychosocial needs

\author{
Evaluation of a cognitive-behavioural \\ self-management group programme
}

Esther STF Smeulders 
The studies presented in this thesis were performed at Maastricht University/CAPHRI School for Public Health and Primary Care. CAPHRI School for Public Health and Primary Care participates in the Netherlands School of Primary Care Research (CaRe), which has been acknowledged since 1995 by the Royal Netherlands Academy of Arts and Sciences (KNAW).

ISBN: 978-90-9024966-7

Cover art: 'Vortex' by Tim Stringer

Printed by: Drukkerij Quickprint, Nijmegen

(c) Copyright, Esther S.T.F. Smeulders, 2009

All rights reserved. No part of this thesis may be reproduced, stored in a retrieval system, or transmitted, in any form or by any means, electronic, mechanical, photocopying, recording, or otherwise, without written permission from the author or from the publisher holding the copyright of the published articles. 


\title{
Heart failure self-management: balancing between medical and psychosocial needs
}

\author{
Evaluation of a cognitive-behavioural \\ self-management group programme
}

\author{
PROEFSCHRIFT \\ ter verkrijging van de graad van doctor \\ aan de Universiteit Maastricht, \\ op gezag van de Rector Magnificus, \\ Prof. mr. G.P.M.F. Mols, \\ volgens het besluit van het College van Decanen, \\ in het openbaar te verdedigen op \\ vrijdag 26 februari 2010 om 12.00 uur \\ door
}

Esther Sophia Theodora Francisca Smeulders 


\section{Promotores:}

Prof. dr. G.I.J.M. Kempen

Prof. dr. J.Th.M. van Eijk

\section{Copromotor:}

Dr. J.C.M. van Haastregt

\section{Beoordelingscommissie:}

Prof. dr. H.W. van den Borne (voorzitter)

Dr. T. Jaarsma (Universitair Medisch Centrum Groningen)

Dr. P.M.J.C Kuijpers

Prof. dr. J.P.J. Slaets (Universitair Medisch Centrum Groningen)

Prof. dr. R. de Wit

The research described in this thesis was supported by a grant of the Netherlands Heart Foundation (NHF-2002B005).

Financial support by the Netherlands Heart Foundation for the publication of this thesis is gratefully acknowledged. 
And I was thinking about how life can sometimes be like a vortex, pulling you in, out of control. It is only when we realize that we have far less control over life than we often want and the "ride" should be enjoyed for itself. 



\section{Contents}

Chapter 1 General introduction

Chapter 2 Nurse- and peer-led self-management programme for patients with an implantable cardioverter defibrillator; a feasibility study

Chapter 3 Evaluation of a self-management programme for congestive heart failure patients: design of a randomized controlled trial

Chapter 4 Effects of a nurse-led self-management group programme among congestive heart failure patients: a randomized controlled trial

Chapter 5 The impact of a self-management group programme on health behaviour and healthcare utilization among congestive heart failure patients

Chapter 6 Heart failure patients with a lower educational level and better cognitive status benefit most from a self-management group programme

Chapter 7 Feasibility of a group-based self-management programme among congestive heart failure patients

Chapter 8 General discussion

Samenvatting

Co-authors and affiliations

Dankwoord 



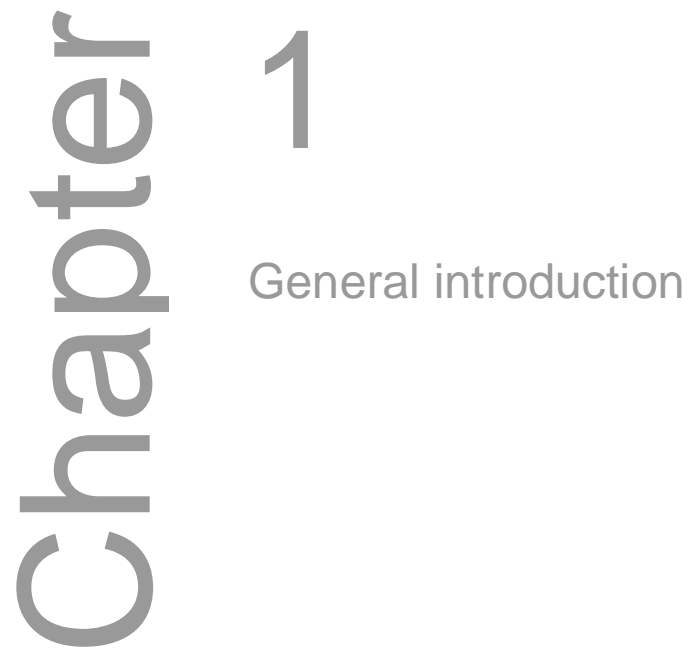




\section{The congestive heart failure challenge}

Managing congestive heart failure (CHF) is an individual and a societal challenge. CHF is a syndrome in which patients have symptoms of shortness of breath and/or fatigue, show signs of fluid retention and have an abnormality of the heart at rest. ${ }^{1}$ In $70 \%$ of patients CHF is caused by coronary heart disease. ${ }^{1}$ CHF is a progressive disorder threatening daily functioning and resulting in reduced life expectancy. ${ }^{1-4}$ Overall, $40 \%$ of $\mathrm{CHF}$ patients admitted to the hospital are readmitted or even die within one year. Overall, $50 \%$ of CHF patients die within four years. ${ }^{1}$ The prevalence of $\mathrm{CHF}$ is estimated at $1-3 \%$ in the western world, with incidences approaching 5-10 per 1000 people per year. ${ }^{1,5,6}$ Age is an important factor affecting these numbers. In persons older than 50 years the prevalence and incidence of $\mathrm{CHF}$ increase progressively. ${ }^{1,5,6}$ The mean age of CHF patients in the western world is 75 years, and the prevalence of CHF in 70 to 80 -year-olds reaches $10-20 \%$., The CHF burden is expected to increase substantially over at least the next two decades. ${ }^{7}$ A steady increase in the number of CHF patients and hospital admissions for $\mathrm{CHF}$ is predicted because of the ageing population, success in prolonging survival in patients suffering coronary events, and success in postponing coronary events by effective primary and secondary prevention. ${ }^{1,5-9}$ These changes in the age structure of the population and improved treatment options will further fuel the CHF epidemic in western societies and impose an enormous burden on society in terms of CHF-associated healthcare costs. ${ }^{1,5}$ In Europe, CHF already accounts for about $2 \%$ of national expenditure on healthcare, mostly due to the cost of hospital admissions. ${ }^{1,10}$

CHF substantially affects patients' quality of life as it impacts daily life in terms of physical, social and emotional functioning. ${ }^{4}$ Several studies indicate that quality of life may be even more severely impaired by CHF than by other highly prevalent chronic diseases such as chronic obstructive pulmonary disease (COPD), arthritis, or angina. ${ }^{11-16}$ Regarding physical functioning, the exercise capacity of $\mathrm{CHF}$ patients is often reduced due to cardiac dysfunction and symptoms of breathlessness and fatigue. ${ }^{17,18}$ Consequently, patients become physically impaired and lose independence as daily activities can no longer be performed to the same level as before CHF diagnosis. ${ }^{4,19,20}$ In addition, patients can no longer fulfil the demands of their former social life as these physical limitations hinder the accomplishment of personal and professional roles. Patients may no longer be able to work or participate in their social network. ${ }^{19,20} \mathrm{CHF}$ can also have a profound impact on the relationship between patients and their partners, for example, in terms of role changes and sexual disability. ${ }^{20-22}$ In addition, CHF patients may experience communication problems because of conflicting opinions with their partner 
regarding healthy lifestyles or because of memory and attention deficits. ${ }^{20,21,23}$ Finally, apart from reporting physical and social limitations, many CHF patients suffer psychological distress as they are emotionally burdened by the fear of dying and by emotions such as anxiety, depression, anger, and hopelessness. ${ }^{12,19,20,22}$ Depressive symptoms, for example, may have a greater impact on CHF patients' quality of life than severity of cardiac dysfunction or functional impairment. ${ }^{16}$ Overall, poor quality of life is associated with hospital readmissions and mortality in CHF patients. ${ }^{13,24,25}$

\section{Treating CHF}

Preventing the progression of $\mathrm{CHF}$ is an essential goal of medical treatment. ${ }^{1}$ Acute worsening of CHF may occur within 24 hours in terms of acute pulmonary oedema, cardiogenic shock, or decompensation of CHF. ${ }^{5}$ Second, CHF treatment aims to improve quality of life by helping the patient live an independent life, reducing unpleasant symptoms, and avoiding hospital admissions. ${ }^{1,26}$ Both pharmacological and non-pharmacological strategies are included in CHF treatment. ${ }^{1,27}$

$\mathrm{CHF}$ treatment regimen depends on factors such as disease severity and type of cardiac dysfunction. CHF severity is classified by the New York Heart Association (NYHA) classification, varying from NYHA Class I, where patients are not limited by dyspnoea or fatigue, to NYHA Class IV, where patients are unable to continue physical activity without discomfort as they are markedly symptomatic and need frequent medical attention. ${ }^{1}$ Regarding type of cardiac dysfunction a distinction is frequently made between systolic and diastolic $\mathrm{CHF}$ on the basis of impaired or preserved left ventricular ejection fraction (LVEF) respectively. CHF with preserved LVEF is more common in older people, women and those with hypertension or diabetes, and carries a better prognosis than CHF caused by impaired LVEF. ${ }^{1,5}$ However, as most CHF patients have evidence of both systolic and diastolic dysfunction at rest or during exercise, the distinction between both types of $\mathrm{CHF}$ is considered somewhat arbitrary. ${ }^{1,5,28-31}$ Finally, in CHF treatment, patients' selfmanagement plays a crucial role as it may have a significant impact on symptoms, functional capacity, well being, morbidity and prognosis of $\mathrm{CHF}{ }^{1}$ 


\section{The patient's challenge}

Living with CHF is challenging for many reasons. First, as CHF is a progressive disorder it changes patients' life expectations and causes uncertainty about the future. Second, a reduction in physical, social, and emotional functioning is an important consequence of CHF for patients' daily lives. ${ }^{4}$ Third, for many patients it is rather a challenge adhering to their complex treatment protocols in daily life. Besides adhering to prescribed medications, CHF patients need to adopt considerable lifestyle changes such as adhering to a dietary sodium and fluid restriction, stopping smoking, limiting alcohol intake, and staying physically active. Finally, patients have to recognize the symptoms of CHF deterioration and monitor their weight regularly. ${ }^{1,32-35}$ This demands knowledge, cooperation, and active participation from CHF patients. ${ }^{36}$ Unfortunately, many patients fail to adhere to the treatment regimen as treatment goals are not being achieved. ${ }^{36} \mathrm{~A}$ downward spiral of functional deterioration and non-adherence may then lead to increased healthcare utilization and a progressive decline in the condition of the patient. ${ }^{33,36-38}$

Medication non-adherence is common in about $40-60 \%$ of $\mathrm{CHF}_{\text {patients }}{ }^{39}$ and plays a major role in preventable hospital readmissions. ${ }^{39,40} \mathrm{~A}$ range of factors prevent $\mathrm{CHF}$ patients adhering including socioeconomic factors (e.g. low educational level), healthcare-related factors (e.g. poor patient-provider relationship), condition-related factors (e.g. symptom severity, anxiety, and depression), treatment-related factors (e.g. perceived beneficial effects or side effects of medication), and patient-related factors (e.g. patient forgetfulness). ${ }^{39}$ In addition to medication non-adherence a significant proportion of patients fail to adhere to the non-pharmacological regimen in terms of monitoring weight $(20-80 \%)$, exercising $(9-53 \%)$ and following sodium restrictions $(20-71 \%) .{ }^{36}$ Furthermore, recognizing symptoms of deterioration remains a challenge for patients because of poor CHF knowledge. Recognition of CHF deterioration is crucial to minimise exacerbations and hospital readmissions. ${ }^{27,37,41}$

As a consequence, poor adherence to the treatment regimen and poor symptom recognition may be responsible for over one-third of hospital readmissions in CHF. ${ }^{1,42,43}$ In addition, non-adherence to CHF treatment may be even more urgent among older patients as CHF management in the elderly is often complicated by the presence of comorbidity, polypharmacy, psychosocial problems and age-related problems such as cognitive impairment. $26,38,44-46$ 


\section{Taking control: the role of self-management}

Effective self-management is crucial for CHF patients as it may prevent $\mathrm{CHF}$ exacerbations and hospital readmissions. Therefore, patients are engaged in improving their abilities to manage their chronic condition on a day-to-day basis. ${ }^{34,36,47}$ A variety of terminology is used to describe patients' abilities to manage their chronic condition. Terms such as self-management, self-care, self-monitoring and adherence are used interchangeably, of which selfmanagement and self-care are consistently used as synonyms. ${ }^{34,35}$ This seems justified as most definitions of self-management and self-care put forward over the past few decades emphasize patient participation in managing a chronic condition. According to one of the first definitions, selfmanagement indicates patients' active participation in (chronic) disease treatment. ${ }^{34,48,49}$ For CHF patients self-management implies being able to accept responsibility for managing one or more aspects of the follow-up treatment (e.g. symptom and weight monitoring) ${ }^{50}$ This is in line with $\mathrm{CHF}$ self-care as defined by the European Society of Cardiology (ESC). The ESC state self-care includes "actions aimed at maintaining physical stability, avoidance of behaviour that can worsen the condition and detection of the early symptoms of deterioration". 1,32

Some researchers, however, suggest that self-management extends beyond self-care (in terms of merely following prescribed instructions) and refers to autonomous patient involvement in managing a chronic condition. ${ }^{36,51,52}$ Self-management is then more strongly perceived as cognitive decision-making in which patients recognize and evaluate changes in CHF signs and symptoms and act on these changes by implementing and evaluating treatment strategies. ${ }^{35,36,53}$ A broader perspective on selfmanagement is that living with a chronic condition involves not only the medical management of the disease (i.e. adhering to the treatment regimen) but also role management (i.e. maintaining, changing and creating new meaningful behaviours or life roles to stay socially active), and emotional management (i.e. dealing with the emotional consequences of having a chronic condition). ${ }^{49,54}$ In this definition, not only the medical aspects of coping with a chronic condition are addressed, but emphasis is also given to the psychosocial consequences of being chronically ill.

$\mathrm{CHF}$ self-management exceeds managing only the medical consequences of the disease. As in daily life patients also face considerable difficulties in dealing with the psychosocial consequences of $\mathrm{CHF}$ in terms of social isolation, lack of social support, anxiety, depression, and lack of perceived control. ${ }^{13,55}$ In addition, these psychosocial factors affect patient adherence and quality of life and, therefore, may be considered as important 
as traditional physiologic factors for recurrent hospital readmissions and mortality. ${ }^{13,56,57}$ In this thesis, we use the term self-management and define it as "autonomous and active patient management of the medical/physical (i.e. adherence to the pharmacological and non-pharmacological treatment regimen) and social and emotional consequences of CHF, which aims to improve physical, social and emotional functioning".

\section{Self-management interventions}

In the past ten years, much research has been conducted to improve CHF outcomes by enhancing patients' self-management. McAlister and colleagues $^{58,59}$ carried out two reviews on the effectiveness of 29 interventions on mortality and hospitalization outcomes among CHF patients discharged from hospital. They defined four types of interventions: (1) multidisciplinary specialized follow-up in heart failure clinics; (2) multidisciplinary specialized follow-up in non-clinic settings; (3) telephone follow-up and attendance with a primary care physician; and (4) programmes that primarily focused on enhancing patient self-management activities. All programmes comprised (nurse-led) patient education. A majority of these programmes also included support or counselling to encourage (medical) self-management. Programmes primarily focused on enhancing patient self-management activities significantly reduced hospitalization, but did not affect mortality. Programmes involving CHF care by a multidisciplinary team were found most effective. ${ }^{59}$ $\mathrm{Yu}$ and colleagues ${ }^{60}$ studied the effectiveness of 21 interventions on mortality and hospitalization outcomes among older CHF patients (mean age $\geq 60$ years). A majority of the programmes encouraged (medical) selfmanagement. They concluded that effective CHF interventions for older patients, among other components, should include both intensive patient education and a self-management supportive strategy. ${ }^{60}$ Finally, Jovicic and colleagues $^{50}$ conducted a review of six self-management interventions in which patients were the primary decision-makers. These self-management programmes showed a significant decrease in hospital readmissions, but a non-significant pooled effect for mortality was found. ${ }^{50}$ In summary, these results showed consistent evidence of patients' self-management decreasing hospital readmissions.

In addition to mortality and hospitalization outcomes, many selfmanagement programmes aim to improve quality of life among CHF patients. However, these studies show miscellaneous results. In both the reviews by McAlister and colleagues ${ }^{58,59}$ and $\mathrm{Yu}$ and colleagues, ${ }^{60}$ approximately half of 
the interventions with quality of life endpoints were proven effective. In the review by Jovicic and colleagues, ${ }^{50}$ three programmes included quality of life measures, but programme effects for quality of life were not found..$^{50}$ In addition, most self-management programmes focus on managing the medical/physical aspects of CHF, but few intervention programmes have also incorporated the social and emotional aspects of $\mathrm{CHF}$ management. ${ }^{61-63}$ Therefore, no clear conclusions can yet be drawn about the benefits of selfmanagement interventions focusing on managing the medical/physical, social, and emotional consequences of CHF. For this reason, we decided to perform a randomized controlled trial to assess the effects of the Chronic Disease Self-Management Programme (CDSMP) among CHF patients. The CDSMP elaborates on the definition that self-management deals with the medical, social, and the emotional consequences of being chronically ill and teaches patients to take responsibility for the day-to-day management of their chronic health problems on all three levels. ${ }^{49,64-66}$

\section{The Chronic Disease Self-Management Programme}

Since the 1980s, Lorig and colleagues (Stanford University) have been developing and evaluating self-management programmes for people with chronic conditions. The first programmes were condition-specific (e.g. the Arthritis Self-Management Programme $(A S M P)^{67}$ ) whereas the Chronic Disease Self-Management Programme (CDSMP), which was developed subsequently, addresses general management problems for patients with chronic conditions. ${ }^{49,64-66}$ The principal assumption of the CDSMP is that people with different chronic conditions have similar adaptive tasks because they all have to manage the medical and physical consequences of their disease, as well as its impact on their social and emotional lives. In six weekly two-and-a-half-hour sessions, groups of patients with a chronic condition are taught three self-management skills: (1) how to manage the medical consequences of the disease (medical management; e.g. medication adherence and lifestyle changes such as exercising regularly); (2) how to remain socially active in daily life (role management); and (3) how to manage the emotional consequences of being chronically ill (emotional management). With regard to medical management, topics such as medication usage and making a personal exercise plan are addressed. The programme comprises topics such as action-planning and communication skills which may improve patients' social functioning. Finally, topics such as depression management 
and cognitive symptom management techniques focus on emotional management issues.

The CDSMP is based on Bandura's self-efficacy theory. ${ }^{68}$ Self-efficacy refers to the belief that one is capable of performing intended behaviours successfully. ${ }^{68}$ Patients' perceived self-efficacy is a powerful determinant of behavioural change. ${ }^{69}$ In addition, there are indications that low levels of selfefficacy predict functional decline in CHF patients. ${ }^{2}$ To enhance self-efficacy expectancies, the programme incorporates four strategies, namely skills mastery, reinterpretation of symptoms, modelling, and social persuasion. Skills mastery includes goal-setting, making weekly action plans, and feedback, which takes up about $25-35 \%$ of each session. The programme aims to motivate patients to make behaviour changes by setting personal goals and breaking them down into smaller action plans. During each session, the patients in the group are invited to make their own (tailor made) personal action plan, which needs to be achieved before the next session. In the next session, problem-solving takes place if participants failed in accomplishing their action plans. Reinterpreting symptoms deals with modifying people's beliefs about their symptoms to relieve symptom problems. The programme teaches patients to recognize that their experienced symptoms are not only caused by the disease and, therefore, manageable. In addition, negative symptoms can feed on each other to become a vicious cycle. During the programme sessions, cognitive symptom management techniques are taught to manage stress, depression and other negative emotions and help break this vicious cycle. The modelling and social persuasion aspects of the programme support and motivate patients to make these changes in their behaviours and beliefs.

The first evaluation studies published on the CDSMP showed promising results among heterogeneous groups of patients with chronic diseases, including patients with cardiovascular diseases (e.g. coronary artery disease and $\mathrm{CHF}$ ) and patients with chronic lung disease (e.g. asthma and emphysema). ${ }^{64-66}$ In a randomized controlled trial, improvements were found on health behaviour (e.g. duration of exercise and use of cognitive symptom management), health status (e.g. self-rated health and health distress), and healthcare utilization (e.g. number of visits to the hospital and number of nights spent in a hospital) at six months of follow-up. ${ }^{64}$ In two subsequent cohort studies, some evidence was found for sustained effects at 12 and 24 months of follow-up on outcomes such as duration of exercise, use of cognitive symptom management and health distress. ${ }^{65,66}$ In addition, longterm improvements of the programme on patients' perceived self-efficacy were observed. However, regarding healthcare utilization, the effects showed 
in the six-month trial ${ }^{64}$ did not sustain in the long term, while, in contrast with the original trial, the number of visits to physicians significantly reduced in the long term. ${ }^{65,66}$ Based on favourable findings, the CDSMP was then disseminated nationally within the United States. ${ }^{70}$ In the past five years, several international evaluation studies have been additionally conducted in which the CDSMP was applied in both mixed and homogeneous patient groups. ${ }^{71-87}$ In summary, parts of these studies ${ }^{71-79}$ showed comparable results with the earlier evaluation studies by Lorig and colleagues, ${ }^{64-66}$ while other studies showed less favourable results or no effects at all. ${ }^{80-87}$ However, none of these studies were conducted solely among CHF patients, or in a sufficiently large sub-sample of CHF patients. Therefore, based on previous studies, no final conclusions on the effectiveness and feasibility of the CDSMP among CHF patients can be drawn.

\section{Objectives of this thesis}

The main objective of this thesis is to evaluate the CDSMP among CHF outpatients in The Netherlands. As little research has been conducted on the usefulness of the CDSMP in The Netherlands ${ }^{81,82}$ and no evaluative studies have been conducted among CHF patients in particular, we decided to assess both the effectiveness and feasibility of the CDSMP among CHF patients in a two-group randomized controlled trial with twelve months of follow-up after start of the programme. Hence, the two objectives of the study presented in this thesis are:

(1) to assess the effects of the CDSMP on psychosocial attributes, health behaviour, quality of life, and healthcare utilization among CHF patients; and

(2) to assess the feasibility of the CDSMP in terms of (a) performance of the intervention according to protocol, (b) patients' adherence to the intervention, and (c) the opinions of the patients and leaders about the intervention.

\section{Outline of this thesis}

These objectives are addressed in the different chapters of this thesis. Chapter 2 describes the results of an exploratory pilot study conducted before the randomized controlled trial. This pilot study explored the feasibility and 
potential benefits of the CDSMP in a group of ten cardiovascular disease patients who had received an implantable cardioverter defibrillator (ICD).

Chapter 3 describes the design of the trial to assess the effectiveness and feasibility of the CDSMP among Dutch CHF patients. Details about the design of the study, inclusion of patients, randomization procedure, intervention, and data collection for both effect and process evaluation are included.

Chapters 4 and 5 describe the programme effectiveness among $317 \mathrm{CHF}$ patients, which was studied in a two-group randomized controlled trial with twelve months of follow-up after start of the programme. Chapter 4 presents the effects of the CDSMP on psychosocial attributes, self-care behaviour, and quality of life. Chapter 5 presents the effects on health behaviour and healthcare utilization.

After addressing the results on effectiveness of the CDSMP, more indepth analyses are conducted in Chapter 6 to assess which specific patient characteristics determine the impact of the programme on cardiac-specific quality of life.

Chapter 7 addresses the results of the feasibility of the CDSMP among 186 participating CHF patients and 18 programme leaders. We assessed the extent to which the programme was performed according to protocol, patients' adherence to the programme, and the opinions of both patients and leaders about the programme.

Finally, Chapter 8 contains a general discussion about the main findings. Implications for practice in CHF healthcare and for future research are made. 


\section{References}

1. Dickstein K, Cohen-Solal A, Filippatos G, McMurray JJ, Ponikowski P, PooleWilson PA, et al. ESC Guidelines for the diagnosis and treatment of acute and chronic heart failure 2008 The Task Force for the Diagnosis and Treatment of Acute and Chronic Heart Failure 2008 of the European Society of Cardiology. Developed in collaboration with the Heart Failure Association of the ESC (HFA) and endorsed by the European Society of Intensive Care Medicine (ESICM). Eur J Heart Fail 2008.

2. Kempen GI, Sanderman R, Miedema I, Meyboom-de Jong B, Ormel J. Functional decline after congestive heart failure and acute myocardial infarction and the impact of psychological attributes. A prospective study. Qual Life Res 2000;9(4):439-50.

3. van Jaarsveld $\mathrm{CH}$, Ranchor AV, Kempen Gl, Coyne JC, van Veldhuisen DJ, Sanderman R. Epidemiology of heart failure in a community-based study of subjects aged $>$ or $=57$ years: incidence and long-term survival. Eur $J$ Heart Fail 2006;8(1):23-30.

4. van Jaarsveld $\mathrm{CH}$, Sanderman R, Miedema I, Ranchor AV, Kempen GI. Changes in health-related quality of life in older patients with acute myocardial infarction or congestive heart failure: a prospective study. J Am Geriatr Soc 2001;49(8):10528.

5. Mosterd A, Hoes AW. Clinical epidemiology of heart failure. Heart 2007;93(9):1137-46.

6. Thomas S, Rich MW. Epidemiology, pathophysiology, and prognosis of heart failure in the elderly. Clin Geriatr Med 2007;23(1):1-10.

7. Stewart S, Maclntyre K, Capewell S, McMurray JJ. Heart failure and the aging population: an increasing burden in the 21st century? Heart 2003;89(1):49-53.

8. Murdoch DR, Love MP, Robb SD, McDonagh TA, Davie AP, Ford I, et al. Importance of heart failure as a cause of death. Changing contribution to overall mortality and coronary heart disease mortality in Scotland 1979-1992. Eur Heart $J$ 1998;19(12):1829-35.

9. Senni M, Tribouilloy CM, Rodeheffer RJ, Jacobsen SJ, Evans JM, Bailey KR, et al. Congestive heart failure in the community: trends in incidence and survival in a 10-year period. Arch Intern Med 1999;159(1):29-34.

10. Stewart S, Jenkins A, Buchan S, McGuire A, Capewell S, McMurray JJ. The current cost of heart failure to the National Health Service in the UK. Eur J Heart Fail 2002;4(3):361-71.

11. Hobbs FD, Kenkre JE, Roalfe AK, Davis RC, Hare R, Davies MK. Impact of heart failure and left ventricular systolic dysfunction on quality of life: a cross-sectional study comparing common chronic cardiac and medical disorders and a representative adult population. Eur Heart J 2002;23(23):1867-76.

12. Johansson $P$, Dahlstrom $U$, Brostrom $A$. Factors and interventions influencing health-related quality of life in patients with heart failure: a review of the literature. Eur J Cardiovasc Nurs 2006;5(1):5-15.

13. Moser DK. Psychosocial factors and their association with clinical outcomes in patients with heart failure: why clinicians do not seem to care. Eur J Cardiovasc Nurs 2002;1(3):183-8. 
14. Steptoe A, Mohabir A, Mahon NG, McKenna WJ. Health related quality of life and psychological wellbeing in patients with dilated cardiomyopathy. Heart 2000;83(6):645-50.

15. Stewart AL, Greenfield S, Hays RD, Wells K, Rogers WH, Berry SD, et al. Functional status and well-being of patients with chronic conditions. Results from the Medical Outcomes Study. JAMA 1989;262(7):907-13.

16. Carels RA. The association between disease severity, functional status, depression and daily quality of life in congestive heart failure patients. Qual Life Res 2004;13(1):63-72.

17. Clark AL, Sparrow JL, Coats AJ. Muscle fatigue and dyspnoea in chronic heart failure: two sides of the same coin? Eur Heart J 1995;16(1):49-52.

18. Witte KK, Clark AL. Dyspnoea versus fatigue: Additional prognostic information from symptoms in chronic heart failure? Eur J Heart Fail 2008;10(12):1224-8.

19. Evangelista LS, Kagawa-Singer M, Dracup K. Gender differences in health perceptions and meaning in persons living with heart failure. Heart Lung 2001;30(3):167-76.

20. Europe E, Tyni-Lenne R. Qualitative analysis of the male experience of heart failure. Heart Lung 2004;33(4):227-34.

21. Luttik ML, Blaauwbroek A, Dijker A, Jaarsma T. Living with heart failure: partner perspectives. J Cardiovasc Nurs 2007;22(2):131-7.

22. Stull DE, Starling R, Haas G, Young JB. Becoming a patient with heart failure. Heart Lung 1999;28(4):284-92.

23. Bennett SJ, Sauve MJ. Cognitive deficits in patients with heart failure: a review of the literature. J Cardiovasc Nurs 2003;18(3):219-42.

24. Bennett SJ, Pressler ML, Hays L, Firestine LA, Huster GA. Psychosocial variables and hospitalization in persons with chronic heart failure. Prog Cardiovasc Nurs 1997;12(4):4-11.

25. Konstam V, Salem D, Pouleur H, Kostis J, Gorkin L, Shumaker S, et al. Baseline quality of life as a predictor of mortality and hospitalization in 5,025 patients with congestive heart failure. SOLVD Investigations. Studies of Left Ventricular Dysfunction Investigators. Am J Cardiol 1996;78(8):890-5.

26. Rich MW. Management of heart failure in the elderly. Heart Fail Rev 2002;7(1):8997.

27. Horowitz CR, Rein SB, Leventhal H. A story of maladies, misconceptions and mishaps: effective management of heart failure. Soc Sci Med 2004;58(3):631-43.

28. Brutsaert DL. Diastolic heart failure: perception of the syndrome and scope of the problem. Prog Cardiovasc Dis 2006;49(3):153-6.

29. Brutsaert DL, De Keulenaer GW. Diastolic heart failure: a myth. Curr Opin Cardiol 2006;21(3):240-8.

30. Caruana L, Petrie MC, Davie AP, McMurray JJ. Do patients with suspected heart failure and preserved left ventricular systolic function suffer from "diastolic heart failure" or from misdiagnosis? A prospective descriptive study. BMJ 2000;321(7255):215-8.

31. De Keulenaer GW, Brutsaert DL. Diastolic heart failure: a separate disease or selection bias? Prog Cardiovasc Dis 2007;49(4):275-83. 
32. Jaarsma T, Stromberg A, Martensson J, Dracup K. Development and testing of the European Heart Failure Self-Care Behaviour Scale. Eur $J$ Heart Fail 2003;5(3):363-70.

33. Moser DK, Watkins JF. Conceptualizing self-care in heart failure: a life course model of patient characteristics. J Cardiovasc Nurs 2008;23(3):205-218.

34. Riegel B, Dickson VV. A situation-specific theory of heart failure self-care. $J$ Cardiovasc Nurs 2008;23(3):190-6.

35. Deaton C. Outcome measurement: self-management in heart failure. $J$ Cardiovasc Nurs 2000;14(4):116-8.

36. Evangelista LS, Shinnick MA. What do we know about adherence and self-care? $J$ Cardiovasc Nurs 2008;23(3):250-7.

37. Riegel B, Carlson B. Facilitators and barriers to heart failure self-care. Patient Educ Couns 2002;46(4):287-95.

38. van der $\mathrm{Wal} \mathrm{MH}$, Jaarsma $\mathrm{T}$. Adherence in heart failure in the elderly: Problem and possible solutions. Int J Cardiol 2008;125(2):203-8.

39. Wu JR, Moser DK, Lennie TA, Burkhart PV. Medication adherence in patients who have heart failure: a review of the literature. Nursing Clinics of North America 2008;43(1):133-53.

40. Rich MW, Beckham V, Wittenberg C, Leven CL, Freedland KE, Carney RM. A multidisciplinary intervention to prevent the readmission of elderly patients with congestive heart failure. N Engl J Med 1995;333(18):1190-5.

41. Carlson B, Riegel B, Moser DK. Self-care abilities of patients with heart failure. Heart Lung 2001;30(5):351-9.

42. Evangelista LS, Dracup K. A closer look at compliance research in heart failure patients in the last decade. Prog Cardiovasc Nurs 2000;15(3):97-103.

43. van der Wal $\mathrm{MH}$, Jaarsma $\mathrm{T}$, van Veldhuisen DJ. Non-compliance in patients with heart failure; how can we manage it? Eur J Heart Fail 2005;7(1):5-17.

44. De Geest S, Steeman E, Leventhal ME, Mahrer-Imhof R, Hengartner-Kopp B, Conca $A$, et al. Complexity in caring for an ageing heart failure population: concomitant chronic conditions and age related impairments. Eur J Cardiovasc Nurs 2004;3(4):263-70.

45. Vinson JM, Rich MW, Sperry JC, Shah AS, McNamara T. Early readmission of elderly patients with congestive heart failure. J Am Geriatr Soc 1990;38(12):12905.

46. Zuccala G, Marzetti E, Cesari M, Lo Monaco MR, Antonica L, Cocchi A, et al. Correlates of cognitive impairment among patients with heart failure: results of a multicenter survey. Am J Med 2005;118(5):496-502.

47. Riegel B. Foreword: Self-care of heart failure: what is the state of the science? $J$ Cardiovasc Nurs 2008;23(3):187-9.

48. Creer TL, Renne CM, Christian WP. Behavioral contributions to rehabilitation and childhood asthma. Rehabil Lit 1976;37(8):226-247.

49. Lorig KR, Holman H. Self-management education: history, definition, outcomes, and mechanisms. Ann Behav Med 2003;26(1):1-7.

50. Jovicic A, Holroyd-Leduc JM, Straus SE. Effects of self-management intervention on health outcomes of patients with heart failure: a systematic review of randomized controlled trials. BMC Cardiovasc Disord 2006;6:43. 
51. Ekman I, Cleland JG, Swedberg K, Charlesworth A, Metra M, Poole-Wilson PA. Symptoms in patients with heart failure are prognostic predictors: insights from COMET. J Card Fail 2005;11(4):288-92.

52. Shuldham C, Theaker C, Jaarsma T, Cowie MR. Evaluation of the European Heart Failure Self-care Behaviour Scale in a United Kingdom population. J Adv Nurs 2007;60(1):87-95.

53. Riegel B, Carlson B, Glaser D. Development and testing of a clinical tool measuring self-management of heart failure. Heart Lung 2000;29(1):4-15.

54. Corbin JM, Strauss A. Unending work and care: Managing chronic illness at home. San Francisco, California: Jossey-Bass; 1988.

55. Konstam V, Moser DK, De Jong MJ. Depression and anxiety in heart failure. $J$ Card Fail 2005;11(6):455-63.

56. Moser DK, Worster PL. Effect of psychosocial factors on physiologic outcomes in patients with heart failure. J Cardiovasc Nurs 2000;14(4):106-15.

57. Januzzi JL, Jr., Stern TA, Pasternak RC, DeSanctis RW. The influence of anxiety and depression on outcomes of patients with coronary artery disease. Arch Intern Med 2000;160(13):1913-21.

58. McAlister FA, Lawson FM, Teo KK, Armstrong PW. A systematic review of randomized trials of disease management programs in heart failure. Am J Med 2001;110(5):378-84.

59. McAlister FA, Stewart S, Ferrua S, McMurray JJ. Multidisciplinary strategies for the management of heart failure patients at high risk for admission: a systematic review of randomized trials. J Am Coll Cardiol 2004;44(4):810-9.

60. Yu DS, Thompson DR, Lee DT. Disease management programmes for older people with heart failure: crucial characteristics which improve post-discharge outcomes. Eur Heart J 2006;27(5):596-612.

61. Jaarsma T, Halfens R, Tan F, Abu-Saad HH, Dracup K, Diederiks J. Self-care and quality of life in patients with advanced heart failure: the effect of a supportive educational intervention. Heart Lung 2000;29(5):319-30.

62. Riegel B, Carlson B, Glaser D, Romero T. Randomized controlled trial of telephone case management in Hispanics of Mexican origin with heart failure. $J$ Card Fail 2006;12(3):211-9.

63. Shively M, Kodiath M, Smith TL, Kelly A, Bone P, Fetterly L, et al. Effect of behavioral management on quality of life in mild heart failure: a randomized controlled trial. Patient Educ Couns 2005;58(1):27-34.

64. Lorig KR, Sobel DS, Stewart AL, Brown BW, Jr., Bandura A, Ritter P, et al. Evidence suggesting that a chronic disease self-management program can improve health status while reducing hospitalization: a randomized trial. Med Care 1999;37(1):5-14.

65. Lorig KR, Sobel DS, Ritter PL, Laurent D, Hobbs M. Effect of a self-management program on patients with chronic disease. Eff Clin Pract 2001;4(6):256-62.

66. Lorig KR, Ritter P, Stewart AL, Sobel DS, Brown BW, Jr., Bandura A, et al. Chronic disease self-management program: 2-year health status and health care utilization outcomes. Med Care 2001;39(11):1217-23.

67. Lorig K, Holman $\mathrm{H}$. Arthritis self-management studies: a twelve-year review. Health Educ Q 1993;20(1):17-28.

68. Bandura A. Self-efficacy: the exercise of control. New York: Freeman; 1997. 
69. Sherer M, Maddux JE, Mercandante B, Prentice-Dunn S, Jacobs B, Rogers RW. The self-efficacy scale: construction and validation. Psychol Rep 1982;51:663-71.

70. Lorig KR, Hurwicz ML, Sobel D, Hobbs M, Ritter PL. A national dissemination of an evidence-based self-management program: a process evaluation study. Patient Educ Couns 2005;59(1):69-79.

71. Barlow JH, Wright CC, Turner AP, Bancroft GV. A 12-month follow-up study of self-management training for people with chronic disease: are changes maintained over time? Br J Health Psychol 2005; 10(Pt 4):589-99.

72. Fu D, Fu H, McGowan $P$, Shen YE, Zhu L, Yang H, et al. Implementation and quantitative evaluation of chronic disease self-management programme in Shanghai, China: randomized controlled trial. Bull World Health Organ 2003;81(3):174-82.

73. Kennedy A, Reeves D, Bower P, Lee V, Middleton E, Richardson G, et al. The effectiveness and cost effectiveness of a national lay-led self care support programme for patients with long-term conditions: a pragmatic randomised controlled trial. J Epidemiol Community Health 2007;61(3):254-61.

74. Lorig KR, Ritter PL, Gonzalez VM. Hispanic chronic disease self-management: a randomized community-based outcome trial. Nurs Res 2003;52(6):361-9.

75. Lorig KR, Ritter PL, Jacquez A. Outcomes of border health Spanish/English chronic disease self-management programs. Diabetes Educ 2005;31(3):401-9.

76. Richardson G, Kennedy A, Reeves D, Bower P, Lee V, Middleton E, et al. Cost effectiveness of the Expert Patients Programme (EPP) for patients with chronic conditions. J Epidemiol Community Health 2008;62(4):361-7.

77. Siu AM, Chan CC, Poon PK, Chui DY, Chan SC. Evaluation of the chronic disease self-management program in a Chinese population. Patient Educ Couns 2007;65(1):42-50.

78. Swerissen H, Belfrage J, Weeks A, Jordan L, Walker C, Furler J, et al. A randomised control trial of a self-management program for people with a chronic illness from Vietnamese, Chinese, Italian and Greek backgrounds. Patient Educ Couns 2006;64(1-3):360-8.

79. Wright CC, Barlow JH, Turner AP, Bancroft GV. Self-management training for people with chronic disease: an exploratory study. Br J Health Psychol 2003;8(Pt 4):465-76.

80. Chan SC, Siu AM, Poon PK, Chan CC. Chronic disease self-management program for Chinese patients: a preliminary multi-baseline study. Int $J$ Rehabil Res 2005;28(4):351-4.

81. Elzen H, Slaets JP, Snijders TA, Steverink N. Evaluation of the chronic disease self-management program (CDSMP) among chronically ill older people in the Netherlands. Soc Sci Med 2007;64(9):1832-41.

82. Elzen H, Slaets JP, Snijders TA, Steverink N. The effect of a self-management intervention on health care utilization in a sample of chronically ill older patients in the Netherlands. J Eval Clin Pract 2008;14(1):159-61.

83. Farrell K, Wicks MN, Martin JC. Chronic disease self-management improved with enhanced self-efficacy. Clin Nurs Res 2004;13(4):289-308.

84. Goeppinger J, Armstrong B, Schwartz T, Ensley D, Brady TJ. Self-management education for persons with arthritis: Managing comorbidity and eliminating health disparities. Arthritis Rheum 2007;57(6):1081-8. 
85. Griffiths C, Motlib J, Azad A, Ramsay J, Eldridge S, Feder G, et al. Randomised controlled trial of a lay-led self-management programme for Bangladeshi patients with chronic disease. Br J Gen Pract 2005;55(520):831-7.

86. Kendall E, Catalano T, Kuipers P, Posner N, Buys N, Charker J. Recovery following stroke: the role of self-management education. Soc Sci Med 2007;64(3):735-46.

87. Lorig K, Ritter PL, Plant K. A disease-specific self-help program compared with a generalized chronic disease self-help program for arthritis patients. Arthritis Rheum 2005;53(6):950-7. 



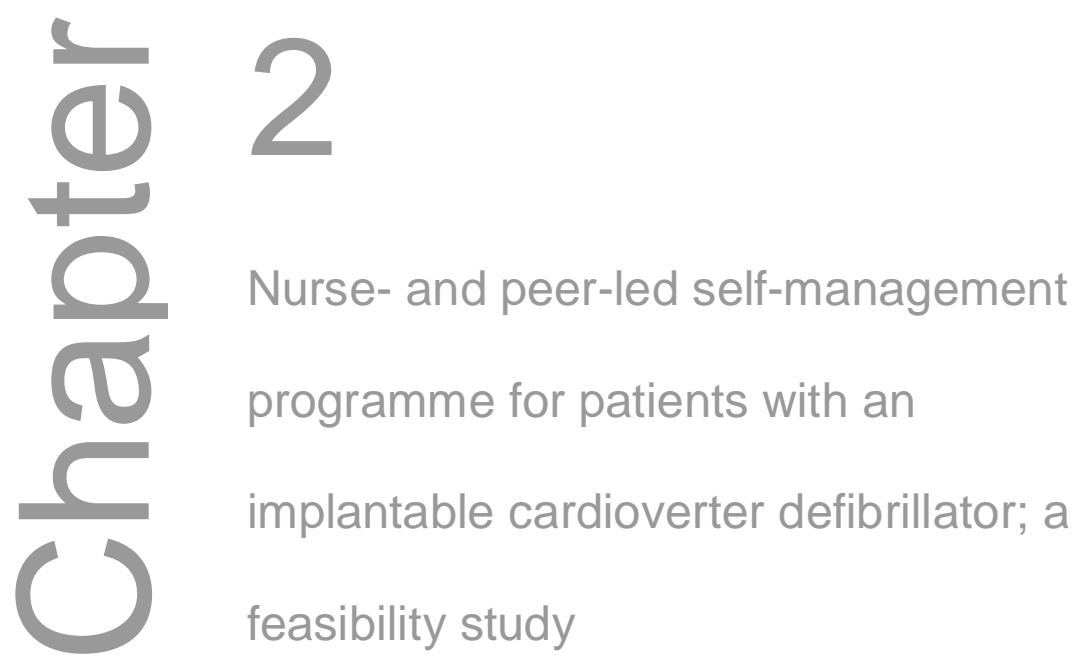

ESTF Smeulders, JCM van Haastregt, BK Dijkman-Domanska, EFM van Hoef, JThM van Eijk, GIJM Kempen

BMC Nursing 2007 19;6:6. 


\section{Abstract}

Background The prevalence of cardiovascular disease (CVD) is increasing. Improved treatment options increase survival after an acute myocardial infarction or sudden cardiac arrest, although patients often have difficulty adjusting and regaining control in daily life. In particular, patients who received an implantable cardioverter defibrillator (ICD) experience physical and psychological problems. Interventions to enhance perceived control and acceptance of the device are therefore necessary. This paper describes a small-scale study to explore the feasibility and the possible benefits of a structured nurse- and peer-led self-management programme (Chronic Disease SelfManagement Programme - CDSMP) among ICD patients.

Methods Ten male ICD patients (mean age $=65.5$ years) participated in a group programme, consisting of six sessions, led by a team comprising a nurse specialist and a CVD patient. Programme feasibility was evaluated among patients and leaders by measuring performance of the intervention according to protocol, attendance and adherence of the participating ICD patients, and patients' and leaders' opinions about the programme. In addition, before and directly after attending the intervention, programme benefits (e.g. perceived control, symptoms of anxiety and feelings of depression, and quality of life) were assessed.

Results The programme was conducted largely according to protocol. Eight patients attended at least four sessions, and adherence ranged from 'good' to 'very good'. On average, the patients reported to have benefited very much from the programme, which they gave an overall report mark of 8.4. The leaders considered the programme feasible as well. Furthermore, improvements were identified for general self-efficacy expectancies, symptoms of anxiety, physical functioning, social functioning, role limitations due to physical problems, and pain.

Conclusions This study suggests that a self-management programme led by a team comprising a nurse specialist and a CVD patient seems feasible according to both patients and leaders. The programme may improve general self-efficacy expectancies, symptoms of anxiety, and quality of life (physical functioning, social functioning, role limitations due to physical problems, and pain) as well. Further investigation of programme effectiveness among a larger sample of ICD patients or other CVD patient groups, is recommended. 


\section{Background}

Worldwide, chronic conditions in general and specifically cardiovascular disease (CVD), are a major health burden. The prevalence of CVD is increasing because of changed life expectancies, unhealthy lifestyles, and improved treatment options. ${ }^{1}$ Because of these improved therapies, patients suffering from acute myocardial infarction have a considerably higher chance of survival. A substantial number of CVD patients receive an implantable cardioverter defibrillator (ICD), making it one of the most important recent advanced therapies in the prevention of sudden cardiac arrest due to lifethreatening arrhythmias. ${ }^{2,3}$

Adjusting to the consequences of CVD and regaining control in daily life can often be difficult, particularly after ICD implantation. Introducing a foreign body into the heart may be considered a major life event; ICD patients may be confronted with a changed body image, problems of psychosocial adaptation, and reduced quality of life. ${ }^{4}$ In addition, symptoms of anxiety and feelings of depression after implantation appear to be common among ICD patients, affecting $24-87 \%$ and $24-33 \%$, respectively, while $13-38 \%$ suffer clinically significant anxiety disorders. ${ }^{5}$ So, although implantation may relieve much of the fear of sudden death, it also introduces new fears, so that the overall effect on psychological health is largely neutral. ${ }^{6}$ Patients have to cope with the presence of a potentially life-threatening condition (i.e. ventricular arrhythmias) and the experience of potentially aversive treatment (i.e. shocks). According to Sears and colleagues, ${ }^{5}$ patients may increasingly limit their range of activities and inadvertently diminish their quality of life due to fear of shocks. To deal with the perceived lack of control, the fundamental psychological challenge for ICD patients is to derive perceived security from the ICD device. ${ }^{5,7}$ Psychological interventions to enhance perceived control and acceptance of the device are therefore necessary. ${ }^{8} \mathrm{~A}$ recent review showed that only a few studies assessed the effects of psychological interventions for ICD patients. ${ }^{9}$ In the majority of these studies, no significant effects of these interventions were found, with exception of a cognitivebehavioural therapy intervention. ${ }^{10}$ In the majority of these studies, the lack of positive results may be explained partly by the small sample sizes and possibly also by the unstructured format of several of the interventions. ${ }^{9}$

Structured self-management programmes have been developed by Lorig and colleagues since the early 1980s, including the widely disseminated Chronic Disease Self-Management Programme (CDSMP). ${ }^{11-16}$ The CDSMP is a generic group-based self-management programme, which is based on the principal assumption that patients with various chronic diseases can learn from each other as they have similar self-management problems and disease- 
related tasks. ${ }^{12}$ The CDSMP, which is generally led by trained lay leaders, has been proved effective in several randomized trials with 6 to 24 months of follow-up. ${ }^{11,12,17}$ It has been shown to significantly improve exercise level and communication with physicians, and to result in positive changes in health status and a reduction of healthcare utilization among heterogeneous groups of patients with chronic conditions (including heart disease, lung disease, stroke, and arthritis). ${ }^{12,13}$ Although the CDSMP is originally developed for use in heterogeneous patient groups, we expect that the programme is also very well applicable to homogeneous patient groups such as ICD patients. The advantages of a homogeneous patient group may be that during the group process patients have better opportunities to share their disease-specific problems, and are more likely to function as role models for each other. The CDSMP is based on Bandura's self-efficacy theory ${ }^{18}$ and incorporates modelling, skills mastery, reinterpretation of symptoms, and social persuasion to enhance self-efficacy expectancies. ${ }^{11,13}$ In cardiac samples, self-efficacy has been associated positively with life style changes and adherence to exercise programs. ${ }^{19}$ In addition, the self-efficacy theory framework has been shown to be suitable for application to ICD patients improving physical and psychological functioning. ${ }^{20}$ As in ICD patients the success of the device implantation may depend, in part, on the recipient's ability to adjust psychologically to the device, ${ }^{21,22}$ it is therefore important to assess the benefits of this self-management programme among ICD patients.

\begin{abstract}
Aim
This study presents the first exploration of the feasibility and the possible benefits of a Dutch nurse- and peer-led version of the structured CDSMP in a small group of ICD patients. To integrate the programme into the Dutch healthcare system, the Dutch version of the CDSMP incorporates a leader team comprising a cardiac nurse specialist ('professional leader') and a CVD patient (i.e. congestive heart failure (CHF) patient; 'peer leader'), instead of two lay leaders.
\end{abstract}




\section{Methods}

\section{Design}

A feasibility study with a one group pre-test/post-test design was carried out in order to assess the feasibility and the possible benefits of the programme.

\section{Participants}

In 2003, a total of 95 patients received an ICD at the University Hospital Maastricht. Of this group, 63 patients living in the three regions nearest the hospital were selected for our study to improve attendance to the programme sessions. Of these 63 patients, 37 persons were excluded due to participation in other studies or because they were no longer treated at the University Hospital Maastricht. This procedure resulted in 26 eligible ICD patients who all received written information about the intervention and the study. Ten ICD patients were able and willing to participate in the study and attended the programme in the period May - June 2004.

\section{Intervention}

The CDSMP consists of six weekly sessions, each lasting two and a half hours, and emphasizes the patients' central role and responsibility in managing their illness. ${ }^{11}$ More specifically, patients learn three types of management tasks to achieve a positive and active management style: patients learn to deal with the medical management of the disease (medical management); to maintain, change and create new meaningful behaviours or life roles (role management), and third, to deal with the emotional consequences of having a chronic condition (emotional management). Regaining a sense of control by learning these three sets of tasks may improve quality of life in patients with a chronic disease ${ }^{13,23}$ and perhaps also in CVD patients receiving ICD therapy. Before the start of the study, the programme was translated into Dutch, including the book Living a healthy life with chronic conditions, ${ }^{24}$ written as a reference source for the material covered in the CDSMP. The patients received this book during the first programme session. Table 1 shows the CDSMP activities per session. 
Table 1: Contents of the CDSMP

\section{Session1}

Activity 1

Activity 2

Activity 3

Activity 4

Activity 5

Activity 6

Session 2

Activity 1

Activity 2

Activity 3

Activity 4

Activity 5

Session 3

Activity 1

Activity 2

Activity 3

Activity 4

Activity 5

Activity 6

Activity 7

Session 4

Activity 1

Activity 2

Activity 3

Activity 4

Activity 5

Activity 6

Activity 7

Session 5

Activity 1

Activity 2

Activity 3

Activity 4

Activity 5

Activity 6

Activity 7

Session 6

Activity 1

Activity 2

Activity 3

Activity 4

Activity 5
Introduction - Identifying common problems

Workshop overview and responsibilities

Differences between acute and chronic conditions Introduction to cognitive symptom management Introduction to action plans

Closing

Feedback/problem-solving session

Dealing with emotions (anger, fear, frustration)

Introduction to exercise

Making an action plan

Closing

Feedback/problem-solving session

Better breathing

Muscle relaxation

Fatigue management

Endurance exercise

Making an action plan

Closing

Feedback/problem-solving/making an action plan

Healthy eating

Distraction

Advance directives for healthcare

Communication skills

Problem-solving

Closing

Feedback/problem-solving/making an action plan

Medication usage

Making informed treatment decisions

Depression management

Self-talk

Guided imagery

Closing

Feedback/problem-solving

Informing the healthcare team

Working with your healthcare professional

Looking back and planning for the future

Closing

Although the CDSMP is originally led by two trained lay leaders, we decided to conduct the programme by teams comprising a cardiac nurse specialist ('professional leader') and a CVD patient (i.e. CHF) ('peer leader'). The choice for a nurse specialist was based on the fact that nurse specialists appear to 
play an important role in promoting self-care behaviour. ${ }^{25-28}$ The CVD patient in the programme acts as a chronic disease role model. Furthermore, because of the exploratory approach of the study, several pairs of leaders were trained. They led the programme sessions in turns, in order to gain sufficient insight in the feasibility of the programme. Before the actual study started, three nurse specialists and four patients received four days of training on the CDSMP protocol. The leaders were trained by one of the researchers (author E.S.) and a cardiac nurse specialist from the University Hospital Maastricht. Both had been instructed as master trainers at Stanford University.

\section{Data collection}

\section{Experiences with the programme}

In order to gather information about the feasibility of the programme, four process outcomes were measured by structured evaluation forms: performance of the intervention according to protocol, attendance and adherence of the patients (i.e. patients' effort during the programme sessions), and patients' and leaders' opinions about the programme.

At the end of each session, the leaders described whether the activities during that session had been carried out according to protocol (i.e. the time limits for each activity adhered to, no activities skipped or shortened, or other training techniques used than required). In addition, the leaders recorded patients' attendance and adherence on a structured form. The overall level of patients' adherence with the programme activities was assessed by the leaders per session using a 5-point scale ('very good' to 'very bad').

The patients' opinions about the programme were assessed after each session and directly after completion of the programme, using structured forms. The patients were asked to give their opinion about the relevance to their personal situation of each session (4-point scale; 'not at all relevant' to 'very relevant') and to give a general report mark for the session (10-point scale; 1 to 10). Directly after completion of the programme, patients were asked to what extent they had benefited from it and which parts they had or had not appreciated. In addition, they were asked to assess the quality of the nurse specialist and the CVD patient as leaders (on a 5-point scale from 'very good' to 'very bad'), to give overall report marks for the leaders and for the programme (10-point scales; 1 to 10), and to suggest possible improvements to the CDSMP. Experiences were assessed only of patients who attended at 
least three sessions. The leaders' opinions about the programme were assessed directly after completion of the six sessions by means of two structured group interviews: one for the professional leaders and one for the peer leaders. At these meetings, the leaders evaluated the protocol's feasibility and the cooperation with the co-leader.

\section{Programme benefits}

In order to assess the possible benefits of the programme, we measured selfefficacy expectancies, perceived control, symptoms of anxiety and feelings of depression, and quality of life at baseline and six weeks follow-up. Sociodemographic variables and comorbidity were assessed once, at baseline.

Self-efficacy expectancies were measured with the 'General Self-Efficacy Scale' (GSES). ${ }^{29}$ Perceived control, or mastery, was measured by means of the Dutch version of the 7 -item scale developed by Pearlin and Schooler. ${ }^{30}$ Symptoms of anxiety and feelings of depression were measured with the Dutch version of the 'Hospital Anxiety and Depression Scale' (HADS) ${ }^{31}$ which is considered to be unbiased by coexisting general medical conditions. ${ }^{32}$ Health-related quality of life was assessed using eight of the nine subscales of the 'RAND 36-item Health Survey' (RAND-36) 33: 'physical functioning', 'social functioning', 'role limitations (due to physical problems)', 'role limitations (due to emotional problems)', 'mental health', 'vitality', 'pain', and 'general health perception'. The psychometric properties of the Dutch versions of the GSES, perceived control scale, the HADS, and the RAND-36 were shown to be satisfactory in previous studies. ${ }^{32,34-37}$

Socio-demographics (sex, age, marital state, living arrangement, educational level, and working situation) and comorbidity (the presence of 19 chronic medical conditions) were assessed at baseline. Data were collected by self-administered questionnaires and telephone interviews. The telephone interviews were conducted independently by trained interviewers.

\section{Ethical considerations}

The Medical Ethics Committee of Maastricht University/University Hospital Maastricht granted approval to conduct this study. All ICD patients were fully informed about the purpose and content of the study. Participation was voluntary and written consent was obtained prior to the measurement at 
baseline (May 2004). The investigation conformed with the principles outlined in the Declaration of Helsinki. ${ }^{38}$

\section{Data analysis}

The data with respect to the experiences with the programme were analysed in a descriptive way. To assess the possible benefits for the ICD patients with respect to the outcome measures, means and standard deviations at baseline and at follow-up were computed. SPSS for Windows, version 12.0.1 was used.

\section{Results}

\section{Patient characteristics}

Patients' mean age was 65.5 years $(S D=7.9)$ and ranged from 50 to 76 . All patients were male. The majority of patients did not live alone $(n=7)$, had a fairly high level of education (six patients had attended first stage of tertiary education) and were unemployed $(n=8)$. The two patients with employment worked at least 32 hours per week. Mean time with the ICD was 8.3 months (range: 5-15 months) before inclusion in the study. The majority of the patients had a primary diagnosis of coronary artery disease $(n=8)$, of whom two patients had been resuscitated prior to ICD implantation because of ventricular arrhythmias. Patients' mean left ventricular ejection fraction was $32 \%$ (range: $20-53 \%$ ), of whom the majority had suffered from at least one myocardial infarction prior to ICD implantation $(n=9)$ and experienced New York Heart Association (NYHA) Class II to III symptoms based on results of exercise tests $(n=8)$. All patients had received medical treatment for their heart condition in the preceding twelve months. With regard to shock history, two patients had experienced shocks in the period from implantation to inclusion in the study; one patient had experienced two shocks, the other patient had experienced an electrical storm of three shocks. Regarding comorbidity, five patients reported no comorbid chronic conditions, while the other five patients had received medical help in the preceding twelve months for at least one comorbid chronic condition. 


\section{Programme feasibility}

\section{Performance according to protocol}

The leaders stated that each session was carried out largely according to protocol. Only minor protocol deviations were reported (i.e. spending more time on a topic than planned) as a result of problems with 'action-planning' and 'problem-solving' (first activity, session two and five). This was due to an imperfect match between the level of some of the action plans and the patients' daily activities. Also, the activities 'advance directives for healthcare' and 'communication skills' (session four) overran their time within the session, because these activities were appraised as difficult topics to deal with.

\section{Patients' attendance and adherence}

With regard to the attendance of the ten patients, the leaders reported that one person withdrew from the programme after the first session because the programme did not meet his expectations. One patient could only attend the first two sessions, because of hospitalization for an ICD device problem. The eight remaining patients attended at least four sessions; one person could not attend the first two sessions because of interference with his work schedule, two persons were absent once due to other appointments, and five persons attended all sessions. The adherence per session and overall adherence was 'good to very good', according to the leaders.

\section{Patients' opinions about the CDSMP}

The patients appraised the programme as 'rather relevant' to 'very relevant' and this opinion hardly differed for the individual sessions. The second and the last sessions were appreciated most by all patients. In the second session, all activities were appreciated equally: from 'feedback/problemsolving session', 'dealing with emotions', 'introduction to exercise', and 'making an action plan' to the opportunity to exchange personal experiences in that particular session. In the sixth session, 'working with your healthcare professional' was considered the most important activity, together with actionplanning in the activity 'looking back and planning for the future'. The report marks per session showed that patients' overall opinion about the programme was positive; the report marks ranged from 7.6 (first session) to 8.3 (second session). 
Seven of the eight patients filled in the evaluation form at the end of the programme. On average, they reported to have 'benefited very much' from the programme and the overall report mark for the programme was 8.4. According to the patients, the best programme activities were: 'making an action plan' and 'problem-solving', cognitive symptom management techniques (e.g. 'better breathing' and 'muscle relaxation'), 'dealing with emotions', and 'advance directives for healthcare'/'making informed treatments decisions'. As regards the leaders, the ICD patients generally evaluated them as 'good', with the professional leader getting a higher score (report mark: 8.5) than the peer leader (report mark: 7.4). The fact that one of the leaders was a CVD patient (i.e. CHF) had no added value for three patients. Finally, the patients made some suggestions for improvement to the CDSMP: three patients recommended discussing more ICD-specific subjects (e.g. ICD technology) or paying more attention to ICD-specific problems in daily life.

\section{Leaders' opinions about the CDSMP}

The leaders $(n=8)$ considered the programme to be feasible. Yet, they sometimes found it difficult to carry out the programme exactly according to protocol (in particular, the activities 'making an action plan', 'problem-solving', and 'advance directives for healthcare'). Furthermore, the peer leaders experienced some role ambiguity and had some difficulties with modelling, which sometimes resulted in the professional leader interfering or taking over parts of the peer leader's activities.

\section{Programme benefits}

As Table 2 shows, the mean scores improved at follow-up, except for three subscales of the RAND-36 (vitality, general perceived health, and role limitations due to emotional problems). The largest differences in mean scores at baseline and at follow-up were found for general self-efficacy expectancies, symptoms of anxiety, and four RAND-36 subscales: physical functioning, social functioning, role limitations due to physical problems, and pain. 
Table 2: Means and standard deviations at baseline and follow-up $(n=10)$

\begin{tabular}{|c|c|c|c|c|}
\hline & \multicolumn{2}{|c|}{ At baseline } & \multicolumn{2}{|c|}{$\begin{array}{l}\text { At follow-up } \\
\text { (6 weeks) }\end{array}$} \\
\hline & Mean & SD & Mean & SD \\
\hline Perceived control* $[7-\underline{35}]$ & 27.1 & 4.1 & 28.1 & 2.5 \\
\hline \multicolumn{5}{|l|}{$\operatorname{HADS}^{\dagger}[\underline{0}-21]$} \\
\hline Symptoms of anxiety & 5.1 & 3.4 & 3.7 & 2.4 \\
\hline Feelings of depression & 3.9 & 3.7 & 2.9 & 3.1 \\
\hline \multicolumn{5}{|l|}{ RAND-36* $[0-100]$} \\
\hline Physical functioning & 66.0 & 17.4 & 72.8 & 22.5 \\
\hline Social functioning & 75.0 & 22.5 & 84.7 & 25.6 \\
\hline Role limitations (physical problem) & 50.0 & 48.4 & 69.4 & 42.9 \\
\hline Role limitations (emotional problem) & 74.1 & 40.1 & 70.4 & 45.5 \\
\hline Mental health & 79.6 & 13.6 & 82.7 & 13.9 \\
\hline Vitality & 63.9 & 22.6 & 63.9 & 18.8 \\
\hline Pain & 80.5 & 22.0 & 86.6 & 20.3 \\
\hline General health perception & 56.7 & 19.5 & 56.7 & 22.9 \\
\hline
\end{tabular}

\section{Discussion}

From this feasibility study we learned that Lorig's structured self-management programme is applicable to a homogeneous group of ICD patients. The CDSMP was feasible according to patients and leaders. The patients appraised the programme as relevant and gave an overall report mark of 8.4. Furthermore, eight of the ten patients attended at least four sessions of the programme and only minor deviations from the protocol were reported. However, the leaders experienced some role ambiguity. This may be due to the fact that the CDSMP protocol does not include specific instructions for dividing the activities between the two leaders. Adding specific instructions in the protocol is therefore recommended, in order to diminish this experienced role ambiguity. Furthermore, becoming more experienced with the programme in practice may probably decrease role ambiguity as well. Next to the applicability of the programme, the CDSMP seemed to positively influence general self-efficacy expectancies, symptoms of anxiety, physical functioning, social functioning, role limitations due to physical problems, and pain. Although obviously our small-scale approach does not allow us to come to final conclusions regarding the effectiveness of the programme, our findings seem promising. 


\section{Future research}

This study presented the first exploration of the feasibility and the possible benefits of the CDSMP among ICD patients. Based on the results of this study, we recommend further investigation of the feasibility and effectiveness of the CDSMP among a larger sample of ICD patients or other CVD patient groups. Because of the exploratory approach of the study, several pairs of leaders led the programme sessions in turns, in order to gain sufficient insight in the feasibility of the programme. Obviously, it is recommended that in future projects each CDSMP class is led by one team of leaders to guarantee continuity of the programme. The turning pairs of leaders might have interfered at some level with the peer leaders' task to be a successful role model, as they could not optimally share information based on their own experiences with the patients in the group. However, to assure continuity of the process for the patients, after each session a structured report of that session was made by the professional leader to inform the other leaders about the group's progress. The fact that some of the patients considered the role of the CVD patient leader as of minor value for the group process might be explained by this discontinuity in leadership.

In the present study, the programme was led by a cardiac nurse specialist and a peer leader (i.e. a CVD patient), instead of two trained lay leaders. This adaptation was made in order to facilitate implementation in regular healthcare in The Netherlands and to control continuity of the programme if the peer leader should experience physical problems due to the disease. Both professional and peer leaders were positive about leading the sessions together. The professional leader could support the peer leader in conducting the programme, where the peer leader could discuss personal experiences with the disease during the sessions. Therefore, in future projects a combination of a professional leader and a peer leader per CDSMP class seems to be a good alternative to a team of two lay leaders. However, the final choice for the most appropriate composition of the teams is dependent on the setting in which it is going to be implemented.

Finally, in addition to assessing the effects of the programme among ICD patients, future studies could also study the effectiveness of the programme among partners of patients, as they experience physical and psychosocial problems as well in the caring process. ${ }^{39}$ The CDSMP protocol allows partners of patients with chronic conditions to attend the programme, though for this feasibility study we decided not to invite partners for logistic reasons. In addition, future research should also assess whether the intervention positively influences healthcare utilization in terms of visits to the outpatient clinic or hospitalization days. 


\section{Conclusions}

The results of this study suggest that a group-based self-management programme led by a team comprising a nurse specialist and a CVD patient is feasible for use among ICD patients. In summary, we may conclude that the intervention was generally performed according to protocol, that the patients' attendance and adherence were high, and that the opinion of both patients and leaders was positive. The experience of running the intervention can therefore be concerned as satisfactory. Furthermore, attending the CDSMP may positively influence general self-efficacy expectancies, symptoms of anxiety, physical functioning, social functioning, role limitations due to physical problems, and pain. As the small-scale approach of this study did not allow testing for statistical inferences, conclusions derived from this study should be taken with some caution. Therefore, we recommend assessing the effectiveness of the programme among a larger sample of ICD patients or other CVD patient groups in a randomized controlled trial.

\section{Acknowledgements}

This research project was funded by the Netherlands Heart Foundation (2002B005) and the University Hospital Maastricht (PF 179), The Netherlands. This study was conducted within CAPHRI School for Public Health and Primary Care of Maastricht University in The Netherlands in cooperation with Lorig and colleagues from Stanford University. We thank N. Steverink, H.A. Elzen, and J.P. Slaets from the University Medical Center Groningen in The Netherlands for their willingness to share Dutch intervention materials. We want to thank Y.J. Stevenhagen for providing medical history information of the participants from the ICD database of the University Hospital Maastricht. 


\section{References}

1. Yach D, Hawkes C, Gould CL, Hofman KJ. The global burden of chronic diseases: overcoming impediments to prevention and control. JAMA 2004;291(21):2616-22.

2. Kamphuis HC, de Leeuw JR, Derksen R, Hauer RN, Winnubst JA. Implantable cardioverter defibrillator recipients: quality of life in recipients with and without ICD shock delivery: a prospective study. Europace 2003;5(4):381-9.

3. Seidl K, Senges J. Worldwide utilization of implantable cardioverter/defibrillators now and in the future. Card Electrophysiol Rev 2003;7(1):5-13.

4. Duru F, Buchi S, Klaghofer R, Mattmann H, Sensky T, Buddeberg C, et al. How different from pacemaker patients are recipients of implantable cardioverterdefibrillators with respect to psychosocial adaptation, affective disorders, and quality of life? Heart 2001;85(4):375-9.

5. Sears SF, Jr., Todaro JF, Lewis TS, Sotile W, Conti JB. Examining the psychosocial impact of implantable cardioverter defibrillators: a literature review. Clin Cardiol 1999;22(7):481-9.

6. Lewin RJ, Frizelle DJ, Kaye GC. A rehabilitative approach to patients with internal cardioverter-defibrillators. Heart 2001;85(4):371-2.

7. Sears SF, Kovacs AH, Conti JB, Handberg E. Expanding the scope of practice for cardiac rehabilitation: managing patients with implantable cardioverter defibrillators. J Cardiopulm Rehabil 2004;24(4):209-15.

8. Sears SF, Jr., Conti JB. Quality of life and psychological functioning of icd patients. Heart 2002;87(5):488-93.

9. Edelman S, Lemon J, Kidman A. Psychological therapies for recipients of implantable cardioverter defibrillators. Heart Lung 2003;32(4):234-40.

10. Kohn CS, Petrucci RJ, Baessler C, Soto DM, Movsowitz C. The effect of psychological intervention on patients' long-term adjustment to the ICD: a prospective study. Pace 2000;23(4 Pt 1):450-6.

11. Lorig KR, Sobel DS, Ritter PL, Laurent D, Hobbs M. Effect of a self-management program on patients with chronic disease. Eff Clin Pract 2001;4(6):256-62.

12. Lorig KR, Sobel DS, Stewart AL, Brown BW, Jr., Bandura A, Ritter P, et al. Evidence suggesting that a chronic disease self-management program can improve health status while reducing hospitalization: a randomized trial. Med Care 1999;37(1):5-14.

13. Lorig KR, Holman H. Self-management education: history, definition, outcomes, and mechanisms. Ann Behav Med 2003;26(1):1-7.

14. Fu D, Ding Y, McGowan P, Fu H. Qualitative evaluation of Chronic Disease Self Management Program (CDSMP) in Shanghai. Patient Educ Couns 2006;61(3):389-396.

15. Chan SC, Siu AM, Poon PK, Chan CC. Chronic disease self-management program for Chinese patients: a preliminary multi-baseline study. Int $J$ Rehabil Res 2005;28(4):351-4.

16. Fu D, Fu H, McGowan $P$, Shen YE, Zhu L, Yang $H$, et al. Implementation and quantitative evaluation of chronic disease self-management programme in Shanghai, China: randomized controlled trial. Bull World Health Organ 2003;81(3):174-82. 
17. Lorig KR, Ritter P, Stewart AL, Sobel DS, Brown BW, Jr., Bandura A, et al. Chronic disease self-management program: 2-year health status and health care utilization outcomes. Med Care 2001;39(11):1217-23.

18. Bandura A. Self-efficacy: the exercise of control. New York: Freeman; 1997.

19. Dougherty CM, Johnson-Crowley NR, Lewis FM, Thompson EA. Theoretical development of nursing interventions for sudden cardiac arrest survivors using social cognitive theory. Adv Nurs Sci 2001;24(1):78-86.

20. Dougherty CM, Thompson EA, Lewis FM. Long-term outcomes of a telephone intervention after an ICD. Pace 2005;28(11):1157-67.

21. Beery T, Baas L. Medical devices and attachment: holistic healing in the age of invasive technology. Issues Ment Health Nurs 1996;17(3):233-43.

22. Beery TA, Baas LS, Matthews H, Burroughs J, Henthorn R. Development of the implanted devices adjustment scale. Dimens Crit Care Nurs 2005;24(5):242-8.

23. Lorig K. Self-management in chronic illness. In: Funk SG, Tornquist EM, Leeman J, Miles MS, Harrell JS, editors. Key aspects of preventing and managing chronic illness. New York: Springer Publishing Company; 2001. p. 35-42.

24. Lorig K, Holman H, Sobel D, Laurent D, Gonzalez V, Minor M. Living a healthy life with chronic conditions: self-management of heart disease, arthritis, diabetes, asthma, bronchitis, emphysema and others. Boulder, Colorado: Bull Publishing Company; 2000.

25. Jaarsma T, Halfens R, Huijer Abu-Saad H, Dracup K, Gorgels T, van Ree J, et al. Effects of education and support on self-care and resource utilization in patients with heart failure. Eur Heart J 1999;20(9):673-82.

26. Beswick AD, Rees K, West RR, Taylor FC, Burke M, Griebsch I, et al. Improving uptake and adherence in cardiac rehabilitation: literature review. J Adv Nurs 2005;49(5):538-55.

27. Sol BG, van der Bijl JJ, Banga JD, Visseren FL. Vascular risk management through nurse-led self-management programs. J Vasc Nurs 2005;23(1):20-4.

28. Frich LM. Nursing interventions for patients with chronic conditions. J Adv Nurs 2003;44(2):137-53.

29. Sherer M, Maddux JE, Mercandante B, Prentice-Dunn S, Jacobs B, Rogers RW. The self-efficacy scale: construction and validation. Psychol Rep 1982;51:663671.

30. Pearlin LI, Schooler C. The structure of coping. J Health Soc Behav 1978;19(1):221.

31. Zigmond AS, Snaith RP. The hospital anxiety and depression scale. Acta Psychiatr Scand 1983;67(6):361-70.

32. Spinhoven P, Ormel J, Sloekers PP, Kempen GI, Speckens AE, Van Hemert AM. A validation study of the Hospital Anxiety and Depression Scale (HADS) in different groups of Dutch subjects. Psychol Med 1997;27(2):363-70.

33. Hays RD, Sherbourne CD, Mazel RM. The RAND 36-Item Health Survey 1.0. Health Econ 1993;2:217-227.

34. Bosscher RJ, Smit JH, Kempen Gl. Algemene competentieverwachtingen bij ouderen: Een onderzoek naar de psychometrische kenmerken van de Algemene Competentieschaal (ALCOS) [Global expectations of self-efficacy in the elderly: An investigation of psychometric characteristics of the General Self-Efficacy Scale]. Ned Tijdschr Psychol 1997;52(6):239-248. 
35. Kempen GI, Sanderman R, Miedema I, Meyboom-de Jong B, Ormel J. Functional decline after congestive heart failure and acute myocardial infarction and the impact of psychological attributes. A prospective study. Qual Life Res 2000;9(4):439-50.

36. Kempen GI. Psychometric properties of GLAS baseline measures: a pilot study (in Dutch). Groningen, The Netherlands: Northern Centre for Healthcare Research; 1992.

37. van der Zee KI, Sanderman $\mathrm{R}$. Het meten van de algemene gezondheidstoestand met de RAND-36, een handleiding. Groningen: Noordelijk Centrum voor Gezondheidsvraagstukken; 1993.

38. Rickham PP. Human Experimentation. Code of Ethics of the World Medical Association. Declaration of Helsinki. Br Med J 1964;5402:177.

39. Albarran JW, Tagney J, James J. Partners of ICD patients--an exploratory study of their experiences. Eur J Cardiovasc Nurs 2004;3(3):201-10. 



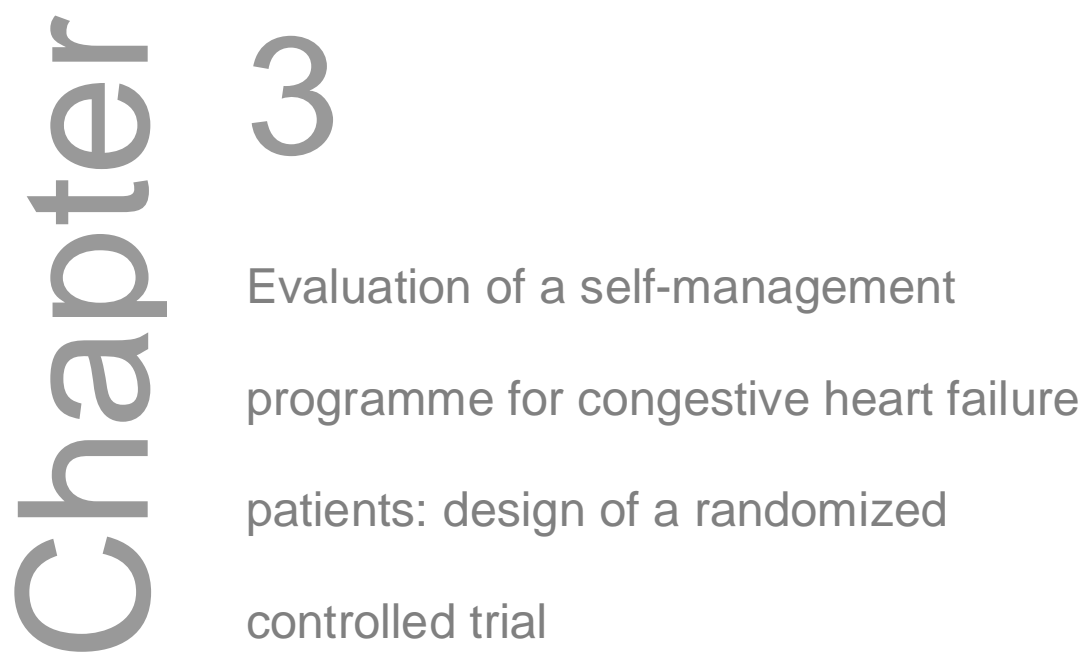

ESTF Smeulders, JCM van Haastregt, EFM van Hoef, JThM van Eijk, GIJM Kempen

BMC Health Services Research 2006 20;6:91. 


\section{Abstract}

Background Congestive heart failure (CHF) has a substantial impact on healthcare utilization and quality of life. It is crucial for patients to cope with CHF adequately, if they are to live an acceptable life. Self-management may play an important role in this regard. Previous studies have shown the effectiveness of the Chronic Disease SelfManagement Programme (CDSMP), a group-based cognitive-behavioural programme for patients with various chronic conditions. However, programme effectiveness has not yet been studied specifically among CHF patients. This paper presents the design of a randomized controlled trial to evaluate the effects of the CDSMP on psychosocial attributes, health behaviour, quality of life, and healthcare utilization among CHF patients.

Methods/Design The programme is being evaluated in a two-group randomized controlled trial. Patients were eligible if they had been diagnosed with CHF and experienced slight to marked limitation of physical activity. They were selected from the heart failure and/or cardiology outpatient clinics of six hospitals. Eligible patients underwent a baseline assessment and were subsequently allocated to the intervention or control group. Patients allocated to the intervention group were invited to attend the self-management programme consisting of six weekly sessions, led by a CHF nurse specialist and a CHF patient. Those allocated to the control group received care as usual. Follow-up measurements are being carried out immediately after the intervention period, and six and twelve months after the start of the intervention.

An effect evaluation and a process evaluation are being conducted. The primary outcomes of the effect evaluation are self-efficacy expectancies, perceived control, and cognitive symptom management. The secondary outcome measures are smoking and drinking behaviour, body mass (Body Mass Index - BMI), physical activity level, self-care behaviour, health-related quality of life, perceived autonomy, symptoms of anxiety and feelings of depression, and healthcare utilization. The programme's feasibility is assessed by measuring compliance with the protocol, patients' attendance and adherence, and the opinions about the programme.

Discussion A total number of 317 patients were included in the trial. At present, follow-up data are being collected. The results of the trial become clear after completion of the data collection in January 2007. 


\section{Background}

Effective management of congestive heart failure (CHF) is one of the major challenges in healthcare today. The number of CHF patients is substantial: CHF affects up to $20 \%$ of the rapidly increasing population of persons aged 65 years and over. Of every three individuals aged 55 years and over, one will develop CHF during their lifetime, with only $35 \%$ surviving five years following diagnosis. ${ }^{1}$ In the aging population of the Western world, this implies a further expansion of the CHF epidemic in the near future. ${ }^{2,3} \mathrm{CHF}$ has a substantial impact on healthcare utilization, daily functioning, and quality of life. ${ }^{4,5}$

There is increasing evidence that self-management programmes for $\mathrm{CHF}$ patients have positive effects on self-care behaviour and quality of life. ${ }^{6-9}$ Managing CHF is a complex task, and comparable to diabetes in that it touches nearly every important aspect of daily life. ${ }^{10}$ It is therefore crucial for patients to cope with $\mathrm{CHF}$ adequately, if they are to live an acceptable life. Exercise intolerance, often experienced by CHF patients, may lead to activity restriction and progressive functional deterioration. This downward spiral may be interrupted by physical activity, which may improve functional capacity and quality of life. ${ }^{11}$ In addition, diet and lifestyle changes, such as limiting salt intake and balancing rest and activity, are important to prevent complications and hospitalization, and to slow the progression of disease. ${ }^{12,13}$ Selfmanagement skills such as fatigue management, breathing techniques, and relaxation can play an important role in everyday CHF management. ${ }^{14}$

To teach patients how to adequately self-manage the consequences of their chronic disease for daily life, Lorig and colleagues developed the Chronic Disease Self-Management Programme (CDSMP) at Stanford University in California (USA). ${ }^{14-18}$ The CDSMP is a structured group-based selfmanagement programme for people with chronic conditions, addressing general management problems for various chronic conditions. The CDSMP is based on the self-efficacy theory developed by Bandura ${ }^{19}$ and incorporates skills mastery, reinterpretation of symptoms, modelling, and social persuasion to enhance self-efficacy expectancies. ${ }^{16,18}$ Self-efficacy expectancies refer to the belief that one can successfully perform intended behaviours, ${ }^{19}$ and are powerful determinants of behavioural change. ${ }^{20}$ There are indications that low levels of self-efficacy expectancies predict functional decline in CHF patients. ${ }^{21}$ Improving self-efficacy expectancies may therefore change health behaviour, improve health-related quality of life, and reduce healthcare utilization among CHF patients.

Previous studies have found favourable effects of the CDSMP (e.g. on self-efficacy expectancies and hospitalization) in arthritis patients ${ }^{22}$ and in groups of patients with various chronic conditions attending the same 
class, ${ }^{17,23}$ even after one and two years. ${ }^{15,16}$ However, the effects of the programme have not yet been studied specifically among CHF patients. Our basic assumption is that increasing levels of self-efficacy expectancies will help CHF patients to interrupt the downward spiral of functional disability and demoralisation after their cardiac event. Therefore, we decided to evaluate the effects of the CDSMP on psychosocial attributes (e.g. self-efficacy expectancies), health behaviour, quality of life, and healthcare utilization of CHF patients. The current paper presents the design of a multi-centre randomized controlled trial evaluating the CDSMP among CHF outpatients in the south of The Netherlands. The study involves an effect evaluation and an evaluation of the intervention process. The process analysis is expected to yield information on factors increasing or hampering the effectiveness and implementation of the programme in outpatient care for CHF patients, which may be crucial later on if the group programme turns out to be effective.

\section{Aims}

The main objectives of the current study are: (1) to assess the effects of the cognitive-behavioural CDSMP intervention on psychosocial attributes, health behaviour, quality of life, and healthcare utilization of CHF patients; and (2) to assess the feasibility of the CDSMP intervention according to patients and leaders of the programme.

\section{Methods/Design}

\section{Design}

This evaluation study is designed as a two-group randomized controlled trial in which patients are randomly allocated to the intervention or control group (the latter receiving usual care). Data are collected at baseline before the start of the intervention, immediately after the programme (six weeks after the start), and six and twelve months after the start of the programme (Figure 1). Data are gathered by means of self-administered questionnaires and telephone interviews. Various measures are taken to ensure blinding in the data collection process. Questionnaires are collected anonymously and sorted by number. The telephone interviews are conducted independently by trained interviewers, who are unaware to which group patients have been allocated. 


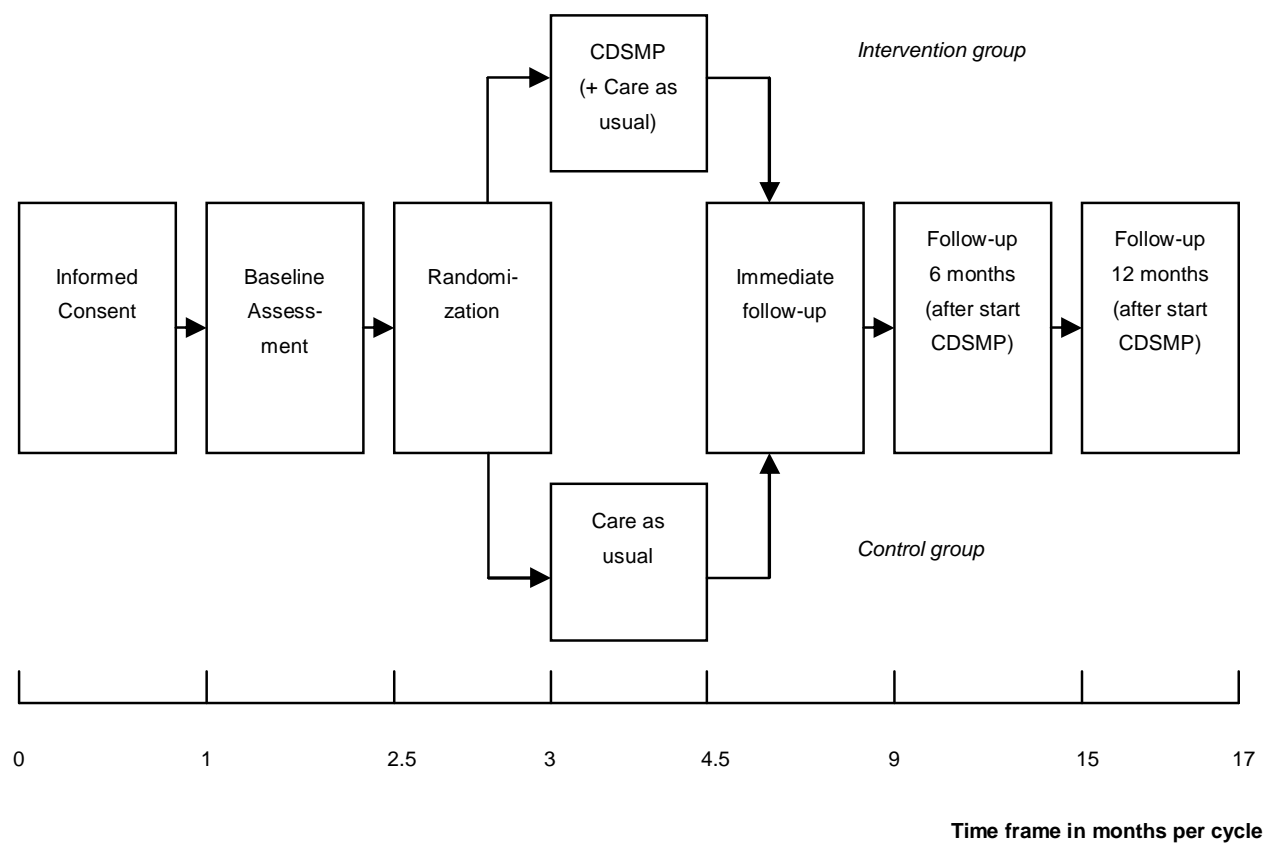

Figure 1: Study design

For practical reasons, the study consists of eleven consecutive cycles, with one or more CDSMP classes in each cycle. In each cycle, patients are included and randomly allocated to the intervention group or control group. The first cycle started in July 2004 and the final one in September 2005. A total of 21 CDSMP classes (with a parallel control group) were conducted in the period October 2004 - January 2006, in six hospitals. At least two CDSMP classes were conducted in each participating hospital (range 2-6). The Medical Ethics Committee of Maastricht University/University Hospital Maastricht granted approval to conduct this trial. Furthermore, local feasibility of the study design was assessed by each of the local Medical Ethics Committees in the other five participating hospitals.

\section{Participants}

Patients were selected by cardiologists or CHF nurse specialists of the heart failure and/or cardiology outpatient clinics in the six participating hospitals. Patients were eligible for the study if they met the following four criteria: (1) having been diagnosed with $\mathrm{CHF}$ based on systolic dysfunction (left ventricular ejection fraction (LVEF) $<40 \%$ with New York Heart Association 
(NYHA) Class II to III symptoms; slight to marked limitation of physical activity) or diastolic dysfunction (NYHA Class II to III), as diagnosed by patients' primary cardiologist, and admitted at least once to the hospital based on cardiac decompensation after CHF diagnosis; (2) having been a CHF patient for at least six months at the start of the intervention period; (3) being able to understand, write, and speak Dutch; and (4) being willing to give informed consent to participate in the study. Patients were excluded if they were participating in other scientific research. All eligible patients received written information about the programme and the study. Patients who signed the informed consent form were invited to participate in the baseline measurement.

\section{Randomization}

In each cycle, patients were randomized after completion of the baseline measurement if a sufficiently large group of eligible patients per hospital could be formed. We decided that at least eight patients per group had to be allocated to the intervention group, because CDSMP classes normally consist of at least eight participants, for reasons of group dynamics. ${ }^{16}$ Eligible patients therefore had a slightly higher chance to be allocated to the intervention group $(59 \%)$ than to the control group $(41 \%)$ during the total recruitment period. The maximum number of patients allocated to each CDSMP class was set at 14 . The randomization procedure was performed by an independent researcher by random computerized allocation using SPSS 12.0 for Windows.

\section{Intervention}

The CDSMP consists of six weekly sessions (two and a half hours per session) and emphasizes patients' central role and responsibility in managing their illness. ${ }^{16}$ The programme aims to teach patients three types of selfmanagement techniques: dealing with the medical management of the disease (medical management); maintaining, changing, and creating new meaningful behaviours or life roles (role management), and dealing with the emotional consequences of having a chronic condition (emotional management). ${ }^{18}$ These self-management techniques are taught by means of skills mastery through weekly action-planning and feedback on progress, modelling of self-management behaviours and problem-solving strategies, reinterpretation of physiological symptoms, and social persuasion through 


\section{group support and guidance for individual self-management efforts. ${ }^{16,18}$ Table 1 shows the CDSMP activities per session.}

\section{Table 1: Contents of the CDSMP}

\section{Session1}

Activity 1

Activity 2

Activity 3

Activity 4

Activity 5

Activity 6

\section{Session 2}

Activity 1

Activity 2

Activity 3

Activity 4

Activity 5

Session 3

Activity 1

Activity 2

Activity 3

Activity 4

Activity 5

Activity 6

Activity 7

Session 4

Activity 1

Activity 2

Activity 3

Activity 4

Activity 5

Activity 6

Activity 7

Session 5

Activity 1

Activity 2

Activity 3

Activity 4

Activity 5

Activity 6

Activity 7

Session 6

Activity 1

Activity 2

Activity 3

Activity 4

Activity 5
Introduction - Identifying common problems

Workshop overview and responsibilities

Differences between acute and chronic conditions

Introduction to cognitive symptom management

Introduction to action plans

Closing

Feedback/problem-solving session

Dealing with emotions (anger, fear, frustration)

Introduction to exercise

Making an action plan

Closing

Feedback/problem-solving session

Better breathing

Muscle relaxation

Fatigue management

Endurance exercise

Making an action plan

Closing

Feedback/problem-solving/making an action plan

Healthy eating

Distraction

Advance directives for healthcare

Communication skills

Problem-solving

Closing

Feedback/problem-solving/making an action plan

Medication usage

Making informed treatment decisions

Depression management

Self-talk

Guided imagery

Closing

Feedback/problem-solving

Informing the healthcare team

Working with your healthcare professional

Looking back and planning for the future

Closing 
While developing the CDSMP, Lorig and colleagues published the book Living a healthy life with chronic conditions, ${ }^{14}$ as a reference source for the material covered in the sessions. The patients received this book at the first session of the programme. Before the start of the study, the original programme had been translated into Dutch, including Living a healthy life with chronic conditions. ${ }^{14}$ Some minor adjustments were made in this translated version of the reference book, based on differences between the American and Dutch settings (e.g. with regard to advance directives).

In the original version of the programme, CDSMP classes are led by two trained lay leaders. For the present study, however, we opted for a combination of a 'professional leader' (a CHF nurse specialist) and a 'peer leader' (CHF patient), acting as a role model for the other CHF patients in the class. The reason was that we expect a programme which is led by at least one professional leader to be easier to implement in regular healthcare than a programme led by two lay leaders only. Furthermore, CHF is a serious disease with unexpected exacerbations and intercurrent health problems. This means that peer leaders may only be able to conduct the programme for a limited period, which could form a threat to the continuity of the programme.

In four of the six hospitals, CHF nurse specialists and CHF patients were trained as leaders. These leaders also conducted CDSMP classes in the two other participating hospitals. A total of twelve professionals and eleven CHF patients received four days of training on the CDSMP protocol in the period April 2004 - November 2005. Four of the twelve professional leaders and two of the eleven peer leaders did not implement the programme in the intervention period, due to work, health, or private issues. Each of the groups in which the leaders were trained $(n=4$ to 6$)$ included both professional leaders and peer leaders, who practised together, to provide an optimal training situation. The leader training was provided by one of the researchers (author E.S.) and a CHF nurse specialist of the University Hospital Maastricht in The Netherlands. Both had been instructed as master trainers at Stanford University by Lorig and colleagues in March 2004. The nurse master trainer also conducted several classes in the intervention period.

\section{Measurements in the effect evaluation}

\section{Primary outcome variables}

The primary outcomes of the effect evaluation are psychosocial attributes, conceptualized as self-efficacy expectancies, perceived control, and cognitive 
symptom management. The primary outcomes are assumed to be directly influenced by the self-management programme.

Self-efficacy expectancies were assessed by two scales; one is a generic scale, allowing us to compare our results with other studies, whereas the other is a specific scale for cardiac patients, used to improve the sensitivity to change in our study population. General self-efficacy expectancies were measured by the 'General Self-Efficacy Scale' (GSES), ${ }^{20}$ which determines the extent to which people believe that they can successfully perform a particular behaviour. Cardiac self-efficacy expectancies were measured using the 'Cardiac Self-Efficacy Questionnaire', ${ }^{24}$ which has also been used in other Dutch research among CHF patients. ${ }^{25,26}$

Perceived control was assessed by means of the mastery scale developed by Pearlin and Schooler. ${ }^{27}$ This scale measures "the extent to which one regards one's life chances as being under one's own control, in contrast to being fatalistically ruled". ${ }^{27}$ The psychometric properties of the Dutch versions of the GSES and the perceived control scale had proved satisfactory in previous studies..$^{21,28,29}$

Cognitive symptom management was assessed by the 'Coping with symptoms scale' developed by Lorig and colleagues, ${ }^{30}$ which measures how often respondents use cognitive symptom management techniques when they feel depressed or experience pain or other unpleasant symptoms. The original 6 -item scale was slightly modified into a 5-item scale by omitting the first two items and adding one extra item about the use of breathing techniques as a way to deal with unpleasant symptoms. ${ }^{31}$ Table 2 provides an overview of the outcome variables of the effect evaluation.

\section{Secondary outcome variables}

The outcome measures considered to be indirectly influenced by the programme (secondary outcomes) are health behaviour, quality of life, and healthcare utilization (Table 2).

Health behaviour was operationalized into three measurements: (1) smoking and drinking behaviour and body mass (Body Mass Index - BMI $\left.\left(\mathrm{kg} / \mathrm{m}^{2}\right)\right)$; (2) physical activity level; and (3) self-care behaviour. Smoking and drinking behaviour were assessed by asking the patients whether they smoked or drank and in what amounts (i.e. mean number of cigarettes/ cigars/pipes smoked per day and mean number of alcoholic drinks per week, respectively). Physical activity level was measured by a modified version of the 'Physical activities scale' developed by Lorig and colleagues. ${ }^{30}$ This scale 
comprises questions about the frequency of walking for exercise, swimming, and bicycling in the preceding month. In addition, respondents were given an opportunity to name alternative exercises in an open-ended question, such as gardening, housekeeping, etc. Finally, CHF-specific self-care behaviour was measured with the 'European Heart Failure Self-Care Behaviour Scale' (EHFScBS), a scale developed in The Netherlands, with good psychometric properties. $^{32}$

Quality of life was conceptualized as health-related quality of life, perceived autonomy, and symptoms of anxiety and feelings of depression. Health-related quality of life was measured using both generic and diseasespecific instruments; the 'RAND 36-item Health Survey' (RAND-36) ${ }^{33}$ and the 'Kansas City Cardiomyopathy Questionnaire' (KCCQ). ${ }^{34}$ Perceived autonomy was assessed by one item using a visual analogue scale to measure to what extent the patients were able to arrange their lives the way they would like to ('I do not feel I can do so at all' to 'I strongly feel I can do so'). Symptoms of anxiety and feelings of depression were measured by the 'Hospital Anxiety and Depression Scale' (HADS), ${ }^{35,36}$ which is considered to be unbiased by coexisting general medical conditions. ${ }^{37}$ The psychometric properties of the Dutch versions of the RAND-36 and the HADS have proved to be satisfactory in previous studies. ${ }^{37,38}$

Finally, healthcare utilization was measured by patients' self-reports on the number of contacts with a general practitioner, cardiologist, other medical specialist, or CHF nurse specialist, the number of hospitalization days, the reason for hospitalization, and the number of visits to accident and emergency departments.

\section{Covariates}

Some additional variables were assessed to characterise the study population and interpret the outcomes of the trial (Table 2). Socio-demographics (sex, age, marital status, living arrangements, educational level, and job situation) were assessed at baseline. In addition, two health-related variables were assessed at baseline: comorbidity using a 19-item checklist of medical conditions $^{21,39}$ and cognitive status using the Dutch version of the Telephone Interview for Cognitive Status (TICS). ${ }^{40,41}$ 
Table 2: Outcome variables of the effect evaluation

\begin{tabular}{|c|c|c|c|c|c|c|}
\hline Variables & $\begin{array}{l}\text { No. of } \\
\text { items }\end{array}$ & Range* $^{\star}$ & BM & FU1 & FU2 & FU3 \\
\hline \multicolumn{7}{|l|}{ Primary outcome variables } \\
\hline General self-efficacy (GSES) [20] & 16 & $16-\underline{80}$ & Q & Q & Q & Q \\
\hline \multicolumn{7}{|l|}{ Cardiac self-efficacy [24] } \\
\hline Control symptoms & 8 & $0-\underline{32}$ & Q & Q & Q & Q \\
\hline Maintain function & 5 & $0-\underline{20}$ & Q & Q & Q & Q \\
\hline Perceived control [27] & 7 & $7-\underline{35}$ & TI & $\mathrm{TI}$ & $\mathrm{TI}$ & $\mathrm{TI}$ \\
\hline Cognitive symptom management [30] & 5 & $0-\underline{25}$ & TI & $\mathrm{TI}$ & $\mathrm{TI}$ & $\mathrm{TI}$ \\
\hline \multicolumn{7}{|l|}{ Secondary outcome variables } \\
\hline Smoking behaviour & 2 & $\mathrm{~N} / \mathrm{A}$ & TI & $\mathrm{TI}$ & $\mathrm{TI}$ & $\mathrm{TI}$ \\
\hline Drinking behaviour & 2 & $\mathrm{~N} / \mathrm{A}$ & TI & $\mathrm{TI}$ & $\mathrm{TI}$ & $\mathrm{TI}$ \\
\hline Body mass & 2 & $\mathrm{~N} / \mathrm{A}$ & $\mathrm{TI}$ & $\mathrm{TI}$ & $\mathrm{TI}$ & $\mathrm{TI}$ \\
\hline Physical activity [30] & 4 & $\mathrm{~N} / \mathrm{A}$ & TI & $\mathrm{TI}$ & $\mathrm{TI}$ & $\mathrm{TI}$ \\
\hline Self-care behaviour (EHFScBS) [32] & 12 & $12-\underline{60}$ & $\mathrm{Q}$ & Q & Q & Q \\
\hline \multicolumn{7}{|l|}{ General quality of life (RAND-36) [33] } \\
\hline Physical functioning & 10 & $0-\underline{100}$ & Q & Q & Q & Q \\
\hline Social functioning & 2 & $0-\underline{100}$ & Q & Q & Q & Q \\
\hline Role limitations (physical problem) & 4 & $0-\underline{100}$ & Q & Q & Q & Q \\
\hline Role limitations (emotional problem) & 3 & $0-\underline{100}$ & Q & Q & Q & Q \\
\hline Mental health & 5 & $0-\underline{100}$ & Q & Q & Q & Q \\
\hline Vitality & 4 & $0-\underline{100}$ & Q & Q & Q & Q \\
\hline Pain & 2 & $0-\underline{100}$ & Q & Q & Q & Q \\
\hline General health perception & 5 & $0-\underline{100}$ & Q & Q & Q & Q \\
\hline Health change & 1 & $0-\underline{100}$ & Q & Q & Q & Q \\
\hline \multicolumn{7}{|l|}{ Cardiac quality of life (KCCQ) [34] } \\
\hline Physical limitations & 6 & $0-\underline{100}$ & Q & Q & Q & Q \\
\hline Symptoms & 8 & $0-\underline{100}$ & Q & Q & Q & Q \\
\hline Self-efficacy & 2 & $0-\underline{100}$ & Q & Q & Q & Q \\
\hline Social interference & 4 & $0-\underline{100}$ & Q & Q & Q & Q \\
\hline Quality of life & 3 & $0-\underline{100}$ & Q & Q & Q & Q \\
\hline Perceived autonomy & 1 & $0-\underline{100}$ & $\mathrm{Q}$ & Q & Q & Q \\
\hline \multicolumn{7}{|l|}{$\begin{array}{l}\text { Symptoms of anxiety and feelings } \\
\text { of depression }[35,36]\end{array}$} \\
\hline Anxiety (HADS-A) & 7 & $\underline{0}-21$ & Q & Q & Q & Q \\
\hline Depression (HADS-D) & 7 & $\underline{0}-21$ & Q & Q & Q & Q \\
\hline Healthcare utilization & 7 & $\mathrm{~N} / \mathrm{A}$ & TI & $\mathrm{TI}$ & $\mathrm{TI}$ & $\mathrm{TI}$ \\
\hline \multicolumn{7}{|l|}{ Covariates } \\
\hline Socio-demographic variables & 9 & $\mathrm{~N} / \mathrm{A}$ & $\mathrm{TI}$ & - & - & - \\
\hline Comorbidity [21, 39] & 19 & $\mathrm{~N} / \mathrm{A}$ & $\mathrm{TI}$ & - & - & - \\
\hline Cognitive status (TICS) $[40,41]$ & 25 & $0-\underline{41}$ & $\mathrm{TI}$ & - & - & - \\
\hline
\end{tabular}

$\mathrm{BM}$ = baseline measurement; FU1 = immediate follow-up; FU2 = follow-up 6 months after the start of the programme; FU3 = follow-up 12 months after the start of the programme; $Q=$ questionnaire; $\mathrm{TI}=$ telephone interview; N/A = not applicable.

* Underlined scores indicate the most favourable scores. 


\section{Measurements in the process evaluation}

To determine the feasibility of the programme, four process outcomes were assessed by means of structured evaluation forms: (1) the extent to which the programme was performed according to protocol and the time spent on the sessions; (2) patients' attendance; (3) overall patient adherence and specific adherence with regard to homework assignments; and (4) the opinions of the patients and leaders about the programme. Table 3 presents the outcome variables of the process evaluation.

With regard to compliance with the protocol, the leaders recorded after each session whether they had exceeded specified time limits per activity, skipped, shortened or added parts to activities, or used different training techniques than specified in the protocol. In addition, they recorded preparation time, travel time, time spent on the session, and activities resulting from the session (e.g. answering patients' questions afterwards).

The leaders reported on patient attendance and adherence at group level. Absent patients were phoned by the professional leader to ask for the reason of their absence. Overall adherence (i.e. patients' efforts during the programme sessions) was also assessed at group level, using a 5-point scale ('very good' to 'very poor'). After completing the programme, both leaders and patients reported on the adherence with regard to homework assignments (e.g. drawing up weekly action plans).

Immediately after the programme, patients who had attended at least three of the six sessions received a structured evaluation form to assess their opinion about the CDSMP. They were asked to what extent they had benefited from the programme (5-point scale; 'not benefited' to 'benefited very much'), and which parts they did or did not appreciate. In addition, patients were asked: (1) to give report marks for the quality of the professional leader and the peer leader, and the programme itself (10-point scale; 1 to 10); (2) whether they would recommend the CDSMP to other CHF patients or to patients with other chronic diseases (e.g. diabetes mellitus); and (3) whether the programme had helped them improve specific health behaviours and coping strategies, compared with the situation before they attended the programme. Finally, the patients were asked to suggest improvements to the CDSMP.

The leaders' opinions about the programme were assessed after each session (report mark on the session, strong and weak points of the session) and after they had completed the programme (report mark on their own skills as leader and questions about the perceived benefits to the patients). At the end of the intervention period (January 2006), all leaders were invited for a structured group interview. During this meeting, the leaders evaluated the 
feasibility of the protocol in more general terms and made recommendations to improve the CDSMP.

\section{Data collection}

Data for the effect evaluation were collected at baseline and the three followup assessments by means of self-administered questionnaires and telephone interviews (Table 2). The telephone interviews were conducted independently by trained interviewers and started two weeks after the questionnaires had been sent out. Patients who announced their intention to stop during the study were asked to complete at least the last follow-up assessment (questionnaire and/or telephone interview).

Data for the process evaluation were collected from the patients by means of self-administered questionnaires. Recording forms and selfadministered questionnaires were used to gather data from the CDSMP leaders (Table 3). In addition, bi-monthly structured meetings were held to allow the leaders to discuss their experiences with the various CDSMP classes.

Table 3: Outcome variables of the process evaluation

\begin{tabular}{|c|c|c|c|}
\hline Process evaluation outcome variables & BDP & FU1 & FU2 \\
\hline \multicolumn{4}{|l|}{ Programme performed according to protocol } \\
\hline Duration of each session & $\mathrm{RF}^{\prime}$ & - & - \\
\hline Deviations from the protocol & $\mathrm{RF}^{\prime}$ & - & - \\
\hline \multicolumn{4}{|l|}{ Attendance by the patients } \\
\hline Reasons for refusal before start of the programme & $\mathrm{TI}^{\mathrm{p}}$ & - & - \\
\hline Number of sessions attended by each patient & $\mathrm{RF}^{\prime}$ & - & - \\
\hline Reasons for stopping during the programme & $\mathrm{Tl}^{\mathrm{P}}$ & - & - \\
\hline \multicolumn{4}{|l|}{ Adherence by patients } \\
\hline Overall adherence & $\mathrm{RF}^{\prime}$ & - & - \\
\hline Adherence regarding homework assignments & - & $Q^{p} / Q^{\prime}$ & - \\
\hline \multicolumn{4}{|l|}{ Opinion about the programme } \\
\hline Extent of benefits from the programme & - & $Q^{p}$ & $Q^{p}$ \\
\hline Strong and weak points of the programme (sessions) & $\mathrm{RF}^{\prime}$ & $Q^{p} / Q^{\prime}$ & - \\
\hline General opinion about the quality of the leaders & - & $Q^{p} / Q^{\prime}$ & - \\
\hline General opinion about the programme & $\mathrm{RF}^{\prime}$ & $Q^{p} / Q^{\prime}$ & - \\
\hline Recommending the programme to other patients & - & $Q^{p}$ & - \\
\hline Programme contributions & - & $Q^{p} / Q^{\prime}$ & $Q^{p}$ \\
\hline Recommendations for improvement & - & $Q^{p} / Q^{\prime}$ & - \\
\hline
\end{tabular}

$\mathrm{BDP}$ = before or during programme; FU1 = immediate follow-up; FU2 = follow-up at 12 months after the programme; $\mathrm{RF}$ = recording form (filled in after each session); $\mathrm{Q}=$ questionnaire; $\mathrm{TI}=$ telephone interview; Data collected from: ${ }^{\prime}=$ leaders $;{ }^{\mathrm{p}}=$ patients. 


\section{Power calculation}

The sample size calculation was based on figures derived from Dutch research among newly diagnosed older $\mathrm{CHF}$ patients ${ }^{21}$ relating to one of the primary outcome variables in the present study, general self-efficacy expectancies (GSES by Sherer and colleagues) ${ }^{20}$. To detect a mean difference of at least 4.0 points in general self-efficacy expectancies between the intervention and control groups ( $S D=11.8$; equivalent to an effect size of 0.34 ), at least 137 patients in both the intervention and control groups were required to achieve a power of $80 \%$ at an alpha of 0.05 . Assuming a drop-out rate of $30 \%$ during the trial, a total of $2 \times 180$ patients have to be included in the study.

\section{Analysis}

Descriptive techniques will be used to characterise the patients participating in the study. Data of the effect evaluation will be analysed according to the intention-to-treat and per-protocol principles. Differences between the intervention and control groups with regard to the primary and secondary outcome variables at the follow-up assessments will be analysed with multivariate techniques adjusting for possible differences in baseline scores and background characteristics. Intervention group patients will be included in the per-protocol analysis if they have attended at least four of the six programme sessions.

Data of the process evaluation will be analysed for patients and leaders by means of descriptive analysis.

\section{Discussion}

\section{Progress of the study}

Between July 2004 and November 2005, a total of 717 eligible patients were selected for inclusion from six heart failure and/or cardiology outpatient clinics, 339 of whom (47.3\%) signed the informed consent form and underwent the baseline assessment (Figure 2). The remaining 378 patients refused or were unable to participate in the study for various reasons: for example, 84 patients did not participate because of physical health problems, 74 patients were not interested or felt no need to participate, and another 74 patients had no time or opportunity to participate (Figure 2). After completion of the baseline 
assessment, 317 patients were included in the trial; 186 patients were allocated to the intervention group and 131 to the control group.

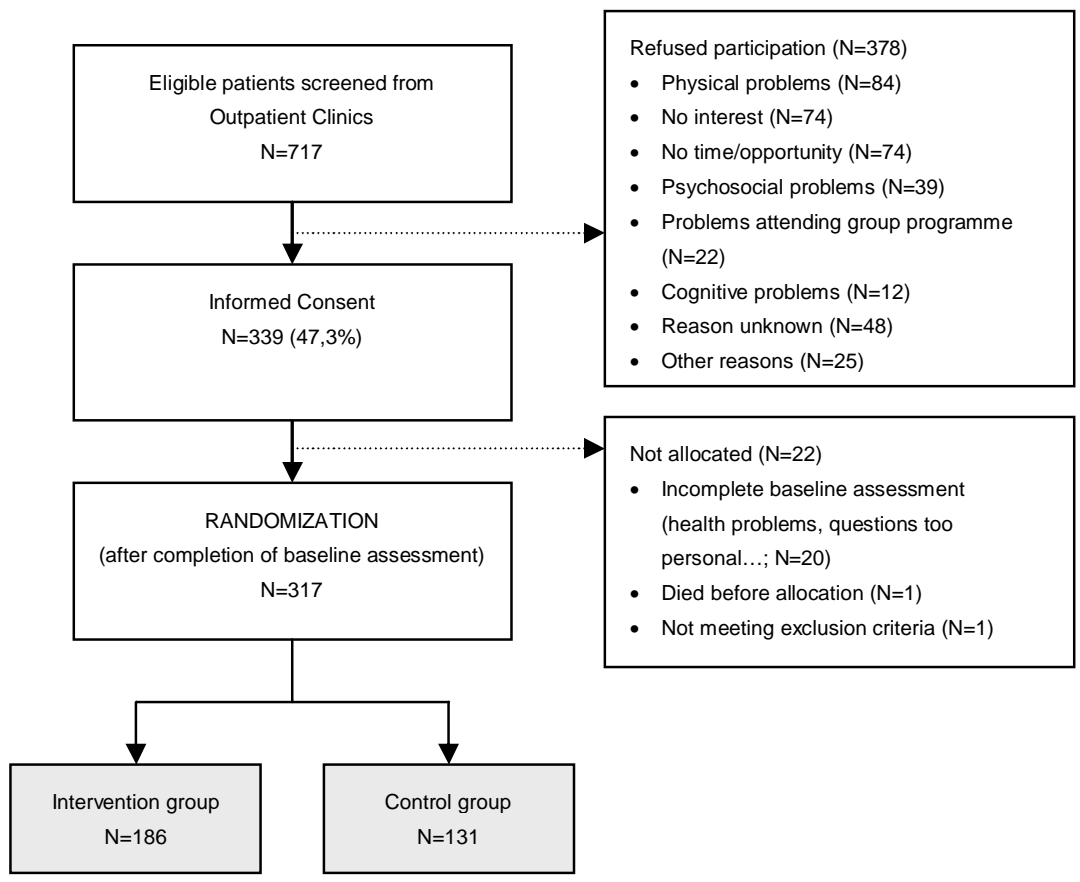

Figure 2: Flow of the patients until randomization

We had calculated that the study would require a sample size of $2 \times 180$, including a $30 \%$ drop-out rate. A total of 186 patients were allocated to the intervention group, which was according to plan. The number of patients allocated to the control group ( $n=131)$, however, was smaller than planned. At present, follow-up data are being collected. The exact drop-out rate and related consequences for the power calculation will become clear after completion of the data collection in January 2007.

\section{Acknowledgements}

This research project was funded by the Netherlands Heart Foundation (2002B005) and the University Hospital Maastricht, The Netherlands (PF 179). This study was conducted within CAPHRI School for Public Health and Primary Care of Maastricht University in The Netherlands, in cooperation with Lorig and colleagues from Stanford University, USA. We would like to thank N. Steverink, H.A. Elzen, and J.P. Slaets of the University Medical Center 
Groningen (The Netherlands) for their willingness to share Dutch programme materials. Finally, we want to thank the University Hospital Maastricht, Atrium Medical Center Heerlen, VieCuri Medical Center Venlo, Laurentius Hospital Roermond, Maasland Hospital Sittard, and Elkerliek Hospital Helmond for participating in the study. 


\section{References}

1. Dickstein $\mathrm{K}$, Jaarsma $\mathrm{T}$. Heart failure management programmes: delivering the message. Eur J Heart Fail 2005;7(3):291-3.

2. Stewart S, Maclntyre K, Capewell S, McMurray JJ. Heart failure and the aging population: an increasing burden in the 21st century? Heart 2003;89(1):49-53.

3. Clark RA, McLennan S, Dawson A, Wilkinson D, Stewart S. Uncovering a hidden epidemic: a study of the current burden of heart failure in australia. Heart Lung Circ 2004;13(3):266-73.

4. van Jaarsveld $\mathrm{CH}$, Sanderman R, Miedema I, Ranchor AV, Kempen GI. Changes in health-related quality of life in older patients with acute myocardial infarction or congestive heart failure: a prospective study. J Am Geriatr Soc 2001;49(8):10528.

5. Blue L, McMurray J. How much responsibility should heart failure nurses take? Eur J Heart Fail 2005;7(3):351-61.

6. DeWalt DA, Malone RM, Bryant ME, Kosnar MC, Corr KE, Rothman RL, et al. A heart failure self-management program for patients of all literacy levels: a randomized, controlled trial [ISRCTN11535170]. BMC Health Serv Res 2006;6:30.

7. Jaarsma T, Halfens R, Huijer Abu-Saad H, Dracup K, Gorgels T, van Ree J, et al. Effects of education and support on self-care and resource utilization in patients with heart failure. Eur Heart J 1999;20(9):673-82.

8. Martensson J, Stromberg A, Dahlstrom U, Karlsson JE, Fridlund B. Patients with heart failure in primary health care: effects of a nurse-led intervention on healthrelated quality of life and depression. Eur J Heart Fail 2005;7(3):393-403.

9. Sethares KA, Elliott $K$. The effect of a tailored message intervention on heart failure readmission rates, quality of life, and benefit and barrier beliefs in persons with heart failure. Heart Lung 2004;33(4):249-60.

10. Schechter CB, Walker EA. Improving adherence to diabetes self-management recommendations. Diabetes Spectr 2002;15(3):170-175.

11. Belardinelli R, Georgiou D, Cianci G, Purcaro A. Randomized, controlled trial of long-term moderate exercise training in chronic heart failure: effects on functional capacity, quality of life, and clinical outcome. Circulation 1999;99(9):1173-82.

12. Moser DK, Watkins JF. Conceptualizing self-care in heart failure: a life course model of patient characteristics. J Cardiovasc Nurs 2008;23(3):205-218.

13. Riegel B, Dickson VV. A situation-specific theory of heart failure self-care. $J$ Cardiovasc Nurs 2008;23(3):190-6.

14. Lorig K, Holman H, Sobel D, Laurent D, Gonzalez V, Minor M. Living a healthy life with chronic conditions: self-management of heart disease, arthritis, diabetes, asthma, bronchitis, emphysema and others. Boulder, Colorado: Bull Publishing Company; 2000.

15. Lorig KR, Ritter P, Stewart AL, Sobel DS, Brown BW, Jr., Bandura A, et al. Chronic disease self-management program: 2-year health status and health care utilization outcomes. Med Care 2001;39(11):1217-23.

16. Lorig KR, Sobel DS, Ritter PL, Laurent D, Hobbs M. Effect of a self-management program on patients with chronic disease. Eff Clin Pract 2001;4(6):256-62. 
17. Lorig KR, Sobel DS, Stewart AL, Brown BW, Jr., Bandura A, Ritter P, et al. Evidence suggesting that a chronic disease self-management program can improve health status while reducing hospitalization: a randomized trial. Med Care 1999;37(1):5-14.

18. Lorig KR, Holman H. Self-management education: history, definition, outcomes, and mechanisms. Ann Behav Med 2003;26(1):1-7.

19. Bandura A. Self-efficacy: the exercise of control. New York: Freeman; 1997.

20. Sherer M, Maddux JE, Mercandante B, Prentice-Dunn S, Jacobs B, Rogers RW. The self-efficacy scale: construction and validation. Psychol Rep 1982;51:663671.

21. Kempen GI, Sanderman R, Miedema I, Meyboom-de Jong B, Ormel J. Functional decline after congestive heart failure and acute myocardial infarction and the impact of psychological attributes. A prospective study. Qual Life Res 2000;9(4):439-50.

22. Lorig K, Gonzalez V. The integration of theory with practice: a 12-year case study. Health Educ Q 1992;19(3):355-68.

23. Lorig KR, Ritter PL, Jacquez A. Outcomes of border health Spanish/English chronic disease self-management programs. Diabetes Educ 2005;31(3):401-9.

24. Sullivan MD, LaCroix AZ, Russo J, Katon WJ. Self-efficacy and self-reported functional status in coronary heart disease: a six-month prospective study. Psychosom Med 1998;60(4):473-8.

25. Arnold R. Quality of life in chronic obstructive pulmonary disease and chronic heart failure: disease-specific and generic factors in adaptation to illness. [PhD thesis]. Groningen: University of Groningen; 2004.

26. Arnold R, Ranchor AV, DeJongste MJ, Koeter GH, Ten Hacken NH, Aalbers R, et al. The relationship between self-efficacy and self-reported physical functioning in chronic obstructive pulmonary disease and chronic heart failure. Behav Med 2005;31(3):107-15.

27. Pearlin LI, Schooler C. The structure of coping. J Health Soc Behav 1978;19(1):221.

28. Bosscher RJ, Smit JH, Kempen Gl. Algemene competentieverwachtingen bij ouderen: Een onderzoek naar de psychometrische kenmerken van de Algemene Competentieschaal (ALCOS) [Global expectations of self-efficacy in the elderly: An investigation of psychometric characteristics of the General Self-Efficacy Scale]. Ned Tijdschr Psychol 1997;52(6):239-248.

29. Kempen GI. Psychometric properties of GLAS baseline measures: a pilot study (in Dutch). Groningen, The Netherlands: Northern Centre for Healthcare Research; 1992.

30. Lorig K, Stewart A, Ritter P, Gonzalez V, Laurent D, Lynch J. Outcome measures for health education and other health care interventions. Thousand Oaks, California: Sage Publications, Inc.; 1996.

31. Elzen H, Slaets JP, Snijders TA, Steverink N. Evaluation of the chronic disease self-management program (CDSMP) among chronically ill older people in the Netherlands. Soc Sci Med 2007;64(9):1832-41.

32. Jaarsma T, Stromberg A, Martensson J, Dracup K. Development and testing of the European Heart Failure Self-Care Behaviour Scale. Eur $J$ Heart Fail 2003;5(3):363-70. 
33. Hays RD, Sherbourne CD, Mazel RM. The RAND 36-Item Health Survey 1.0. Health Econ 1993;2:217-227.

34. Green CP, Porter CB, Bresnahan DR, Spertus JA. Development and evaluation of the Kansas City Cardiomyopathy Questionnaire: a new health status measure for heart failure. J Am Coll Cardiol 2000;35(5):1245-55.

35. Zigmond AS, Snaith RP. The hospital anxiety and depression scale. Acta Psychiatr Scand 1983;67(6):361-70.

36. Bjelland I, Dahl AA, Haug TT, Neckelmann D. The validity of the Hospital Anxiety and Depression Scale. An updated literature review. J Psychosom Res 2002;52(2):69-77.

37. Spinhoven P, Ormel J, Sloekers PP, Kempen GI, Speckens AE, Van Hemert AM. A validation study of the Hospital Anxiety and Depression Scale (HADS) in different groups of Dutch subjects. Psychol Med 1997;27(2):363-70.

38. van der Zee KI, Sanderman R. Het meten van de algemene gezondheidstoestand met de RAND-36, een handleiding. Groningen: Noordelijk Centrum voor Gezondheidsvraagstukken; 1993.

39. Kempen GI, Ranchor AV, Ormel J, van Sonderen E, van Jaarsveld $\mathrm{CH}$, Sanderman R. Perceived control and long-term changes in disability in late middle-aged and older persons: An eight-year follow-up study. Psychol Health 2005;20(2):193-206.

40. Brandt J, Spencer M, Folstein M. The Telephone Interview for Cognitive Status. NNBN 1988;1:111-7.

41. Kempen GI, Meier AJ, Bouwens SF, van Deursen J, Verhey FR. [The psychometric properties of the Dutch version of the Telephone Interview Cognitive Status (TICS)]. Tijdschr Gerontol Geriatr 2007;38(1):38-45. 



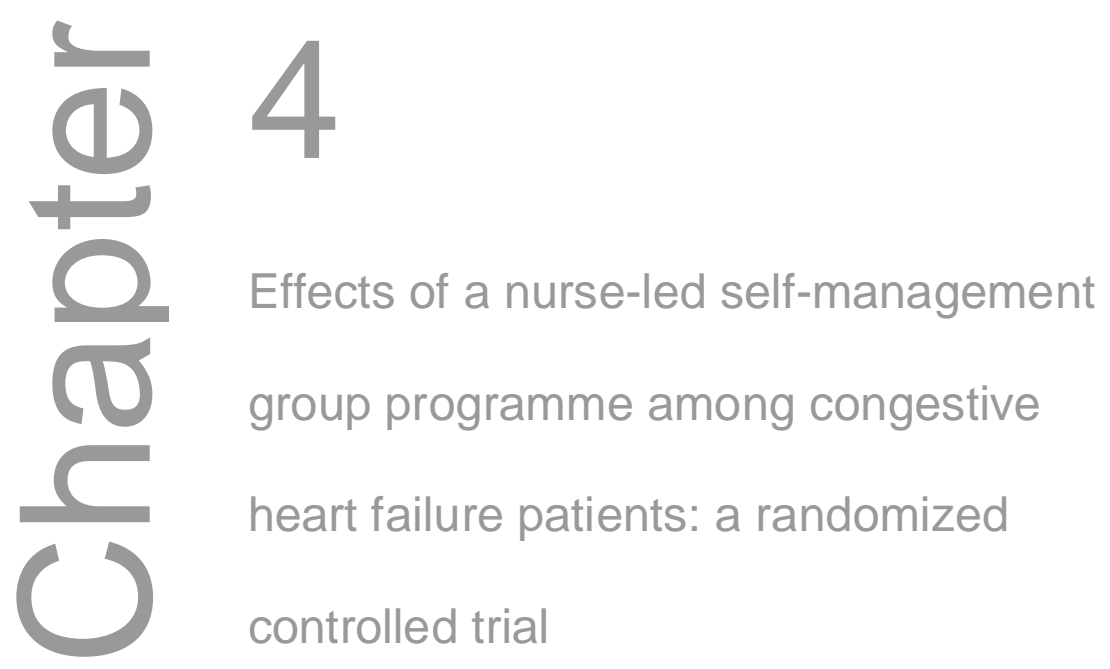

ESTF Smeulders, JCM van Haastregt, T Ambergen, NHKM Uszko-Lencer, JJJ Janssen-Boyne, APM Gorgels, HEJH Stoffers, CLB Lodewijks-van der Bolt, JThM van Eijk, GIJM Kempen

Under review 


\section{Abstract}

Aim To assess the effects of the Chronic Disease Self-Management Programme (CDSMP) on psychosocial attributes, self-care behaviour and quality of life among congestive heart failure (CHF) patients.

Background The CDSMP has been proven effective among different patient groups, however, the effects among CHF patients are unknown. In addition, in the current study cardiac nurse specialists led the programme together with CHF peer leaders, instead of volunteer lay leaders.

Method A randomized controlled trial with twelve months of follow-up after the start of the programme was conducted among 317 patients. Control patients $(n=131)$ received usual care, consisting of regular outpatient checkups. Intervention group patients $(n=186)$ received usual care and participated in the six week selfmanagement group programme. The programme teaches patients to deal with the medical, social and emotional consequences of their disease. Twenty-one classes were conducted in six hospitals in The Netherlands, data were collected between August 2004 and January 2007.

Results Directly after the programme, favourable effects were found for cognitive symptom management, self-care behaviour and cardiac-specific quality of life. No favourable effects were found at six and twelve months after the start of the programme.

Conclusion The CDSMP improved cognitive symptom management, self-care behaviour and cardiac-specific quality of life of patients directly after the programme. No favourable effects were found in the longer term. Future research is necessary to study how long-term effectiveness of the programme among CHF patients can be achieved and how successful adaptations of the programme can be integrated in regular care. 


\section{Introduction}

Ageing of the population in combination with improved treatment options fuel the congestive heart failure (CHF) epidemic, imposing a significant burden on society. ${ }^{1,2}$ On patient level, CHF seriously affects quality of life in terms of physical, social and emotional functioning and reduces patients' life expectancy considerably..$^{3-5}$ As CHF touches nearly every important aspect of daily life, patients are encouraged to take responsibility for their day-to-day disease management. Unfortunately, many CHF patients fail to adhere to the complex regimen. ${ }^{6,7}$ Furthermore, in addition to dealing with the medical aspects of CHF, patients face equal difficulties in dealing with its emotional and social consequences. ${ }^{8-11}$ Psychosocial factors, however, are infrequently treated in CHF care as mainly patients' management of the medical aspects of $\mathrm{CHF}$ is emphasized without addressing social and emotional problems. ${ }^{9}$

\section{Background}

The Chronic Disease Self-Management Programme (CDSMP) teaches patients to deal with the medical (i.e. physical), social and emotional consequences of being chronically ill and emphasizes patients' responsibility in managing their chronic condition on all three levels. ${ }^{12}$ The CDSMP is a generic cognitive-behavioural group programme and is underlined by the assumption that patients with different chronic diseases can learn from each other as they face similar adaptive tasks. ${ }^{13}$ The CDSMP is based on Bandura's self-efficacy theory, in which self-efficacy refers to the confidence to achieve certain behaviours or physiological states under specific conditions. ${ }^{14}$ It is considered a powerful determinant of behavioural change, as self-efficacy beliefs influence how much effort is put forth in changing behaviour and how long people will persevere in the face of obstacles and failures. $^{14,15}$ In addition, there are indications that low levels of self-efficacy predict functional decline among CHF patients. ${ }^{16}$ Previous studies in the USA identified favourable short- and long-term effects of the CDSMP on outcomes such as self-rated health, self-efficacy and use of cognitive symptom management techniques in groups of patients with different chronic conditions. ${ }^{13,17,18}$ Nowadays, the CDSMP is a widely disseminated programme within and outside the USA, applied to both heterogeneous and homogeneous patient groups. ${ }^{19-33}$ Yet, the effectiveness and feasibility of the programme among CHF patients in particular have not been assessed.

We expect that $\mathrm{CHF}$ patients can benefit from this self-management programme, as regarding the medical (i.e. more physical) consequences of 
the disease, CHF patients face many challenges in daily physical functioning, in adhering to a complex regimen and in dealing with end-of-life issues. ${ }^{34,35} \mathrm{In}$ addition, the CDSMP not only emphasizes the medical aspects of selfmanagement, but also provides patients with tools to improve their social and emotional self-management abilities. ${ }^{12,18}$ Increasing self-management skills among CHF patients may positively influence patients' self-care behaviour, their psychosocial attributes related to managing their chronic condition (i.e. self-efficacy expectancies, perceived control, and cognitive symptom management), and their health-related quality of life. The CDSMP led by pairs of cardiac nurse specialists and peer leaders was considered feasible among $\mathrm{CHF}$ patients in a feasibility study which was conducted alongside the trial. ${ }^{36}$

\section{The study}

\section{Aim}

We conducted a randomized controlled trial $(n=317)$ to assess the effects of the CDSMP on psychosocial attributes, self-care behaviour and quality of life among CHF patients who experienced a slight to marked limitation of physical activity.

\section{Design}

A two-group randomized controlled trial with twelve months of follow-up after the start of the programme was conducted in six hospitals in The Netherlands within a range of $86 \mathrm{~km}$ from the study site. The hospitals were comparable on the clinical and outpatient care facilities (i.e. regular checkups with the cardiologist and/or nurse specialist) for CHF patients. Twenty-one CDSMP classes (with parallel control groups) were conducted in the period October 2004 to January 2006 in eleven consecutive cycles, with at least one CDSMP class in each cycle. In each cycle, after completion of the baseline measurement, patients were randomly allocated (per hospital) to the intervention or control group by means of a computerized allocation procedure of SPSS 12.0 for Windows by an independent researcher blinded for patients' characteristics. Control patients received usual care, consisting of regular checkups with the cardiologist and/or the CHF nurse specialist at an outpatient clinic. Patients allocated to the intervention group received the six week self-management group programme in addition to usual care. Patients 
were not blinded for group allocation. For practical reasons, an unequal randomization ratio was applied during the total recruitment period, as in each cycle the time to recruit patients for the study was limited. As the group process is very important in the CDSMP, at least eight participants are required to participate in the classes. ${ }^{18}$ Therefore, based on the total number of recruited patients per cycle, $59 \%$ of the patients were allocated to the intervention group and $41 \%$ to the control group through the full recruitment period to ensure sufficiently large CDSMP classes taking possible attrition from the programme into account. ${ }^{37}$

The sample size estimation was based on figures derived from a Dutch study among newly diagnosed older CHF patients ${ }^{16}$ on general self-efficacy expectancies, ${ }^{15}$ one of the primary outcome variables in the present study. To detect a mean difference of at least 4.0 points between intervention and control groups ( $S D=11.8$; equivalent to an effect size of 0.34 ), a net number of 274 patients needed to be available for longitudinal analyses to achieve a power of $80 \%$ at an alpha of 0.05 . As we assumed a drop-out rate of $25 \%$ during the trial, a total of approximately 360 patients needed to be included in the study. ${ }^{38}$

\section{Participants}

Patients who visited the heart failure and/or cardiology outpatient clinics of the participating hospitals between July 2004 and November 2005 were eligible for the study if they: (1) had been diagnosed with CHF based on systolic dysfunction (left ventricular ejection fraction (LVEF) $<40 \%$, New York Heart Association (NYHA) Class II to III) or diastolic dysfunction (NYHA Class II to III), as diagnosed by patients' primary cardiologist according to the CHF guidelines. ${ }^{39}$ Patients with diastolic dysfunction were eligible after being admitted at least once to the hospital based on cardiac decompensation after CHF diagnosis. In addition, patients were eligible if they: (2) had been a CHF patient for at least six months at the start of the intervention period, after which the CHF diagnosis had been established and therapeutic decisions made; (3) were able to understand, write and speak Dutch; and (4) were willing to give informed consent to participate in the study. Eligible patients received written information about the study and underwent a baseline measurement after signing the informed consent form. Patients were excluded if they were participating in other studies. 


\section{Intervention}

The CDSMP is a structured self-management programme which consists of six weekly group sessions of two and a half hours each. The programme incorporates four strategies to enhance self-efficacy expectancies: skills mastery, reinterpretation of symptoms, modelling and social persuasion. ${ }^{12,13,17,18}$ Skills mastery includes goal-setting and action-planning, which can be considered as core activities of the CDSMP since they take up $25-35 \%$ of each session. Reinterpreting symptoms comprises cognitive symptom management techniques and deals with relieving symptom problems. By modelling and social persuasion, either through group participants or leaders, the patients in the class are expected to become motivated to change their behaviours and beliefs. During the first session, patients received the reference book Living a healthy life with chronic conditions. ${ }^{40}$

In our study, all CDSMP classes were led by a cardiac nurse specialist ('professional leader') and a CHF patient ('peer leader'), instead of two trained lay volunteers. ${ }^{13,17}$ The peer leaders acted as role models for the other patients in the class. Cardiac nurse specialists were included to decrease the potential physical and emotional burden on the peer leaders, to ensure continuity of the programme and to facilitate implementation of the CDSMP in regular Dutch healthcare. All leaders $(n=18)$ were trained according to the CDSMP protocol prior to the intervention period. ${ }^{38}$

\section{Data collection}

We assessed effectiveness of the CDSMP on psychosocial attributes, selfcare behaviour and quality of life. Psychosocial attributes measured were general and cardiac-specific self-efficacy expectancies, perceived control, and cognitive symptom management. General self-efficacy expectancies were measured by the 'General Self-efficacy Scale' (GSES) ${ }^{15}$ and cardiac-specific self-efficacy was measured by the two sub-scales of the 'Cardiac Self-Efficacy Questionnaire'. ${ }^{41}$ Perceived control was measured by a mastery scale developed by Pearlin and Schooler. ${ }^{42}$ The 'Coping with Symptoms Scale,43 was used to measure cognitive symptom management. This scale was modified by omission of the first two items and addition of one extra item about the use of breathing techniques. ${ }^{33}$ Psychosocial attributes were assumed to be directly influenced by the intervention (primary outcomes).

Self-care behaviour and quality of life were considered to be indirectly influenced by the programme (secondary outcomes). Self-care behaviour was measured with the 'European Heart Failure Self-Care Behaviour Scale' 
(EHFScBS). ${ }^{44}$ Regarding quality of life three aspects were assessed: general and cardiac-specific quality of life, perceived autonomy, and symptoms of anxiety and feelings of depression. General quality of life was assessed with the 'RAND 36-item Health Survey' (RAND-36) ${ }^{45}$ and cardiac-specific quality of life was measured with the 'Kansas City Cardiomyopathy Questionnaire' (KCCQ). ${ }^{46}$ Overall summary scores were obtained from both scales. Perceived autonomy was assessed by a visual analogue scale (range 0-100) assessing to what extent the patients were able to arrange their lives the way they would like to. Symptoms of anxiety and feelings of depression were measured by the 'Hospital Anxiety and Depression Scale' (HADS). ${ }^{47}$

Data on the outcome variables were collected confidentially by means of self-administered questionnaires and telephone interviews at baseline, directly after the intervention, and six and twelve months after the start of the intervention, respectively. Socio-demographics (sex, age, educational level, job situation and living arrangements), NYHA classification, number of active diseases (CHF and comorbidities) ${ }^{16}$ and cognitive status ${ }^{48}$ were collected at baseline by telephone as background characteristics. Patients who intended to withdraw during the study were asked to complete at least the last follow-up assessment. Data were collected between August 2004 and January 2007.

\section{Validity and reliability}

To enhance validity and reliability of the data, measures with good psychometric properties were used. The psychometric properties of the Dutch versions of the GSES, perceived control scale, the HADS, and the RAND-36 were shown to be satisfactory in previous studies. ${ }^{44,49-53}$ In addition, the 'Cardiac Self-Efficacy Questionnaire' and 'Coping with Symptoms Scale' were previously used in Dutch studies among older CHF patients. ${ }^{33,54}$ Finally, the telephone interviews were conducted by trained interviewers blinded for group allocation to enhance quality of measurement during the interviews.

\section{Ethical considerations}

Ethics committee approval for conducting this trail was obtained from a university (hospital) to conduct this trial. All patients were fully informed about the purpose and content of the trial. Participation was voluntary and written consent was obtained prior to the measurement at baseline. 


\section{Data analysis}

Background characteristics and outcomes at baseline were compared between the intervention and control groups by means of Chi-square tests, Mann-Whitney $U$ tests and $t$-tests for independent samples. Differences in the outcome variables were analysed with mixed-effects linear regression models with the covariance structure 'unstructured'. We examined between-group differences at the three follow-up assessments using a mixed-model in which group $\times$ time interaction was included. We adjusted for the baseline score on the outcome variable and for background characteristics which differed significantly $(p<0.05)$ between the intervention and control groups at baseline. Data were analysed according to the intention-to-treat principle. If appropriate, effect sizes were calculated by dividing the difference in mean group scores by the pooled standard deviation. Effect sizes of 0.20 were considered small, 0.50 medium and 0.80 large.$^{55}$ In addition, pre-planned perprotocol analyses were performed among control patients and intervention group patients who attended at least four of the six programme sessions. ${ }^{38}$ Missing data were handled according to scale requirements, except for cardiac-specific self-efficacy ${ }^{41}$ where we excluded four items owing to missing values over $25 \%$ at the baseline measurement. We therefore computed one overall score regarding cardiac-specific self-efficacy. All analyses were based on two-sided tests $(p<0.05)$ using SPSS for Windows, version 12.0.1.

\section{Results}

\section{Participants and baseline characteristics}

A total of 717 patients were eligible for the study, of whom $339(47.3 \%)$ signed the informed consent form (Figure 1). After completion of the baseline assessment, 186 patients were allocated to the intervention group and 131 to the control group.

In total, 265 patients (83.6\%) completed the trial (baseline assessment plus at least the twelve-month follow-up measurement) divided into 156 intervention group patients (83.9\%) and 109 control patients (83.2\%). As the recruitment period was limited the target of 274 patients was not completely reached $(n=265 ; 97 \%)$. However, the drop-out rate was less than expected. ${ }^{38}$ Death was the main reason for 'lost to follow-up' in intervention $(n=18)$ and control groups $(n=12)$. No adverse events or side effects were reported during the trial. 
The background characteristics were comparable for intervention and control groups (Table 1). Regarding the outcome measures, patients in the intervention group scored significantly lower on cardiac-specific quality of life at baseline than control patients (Table 3). No other significant differences were found at baseline.

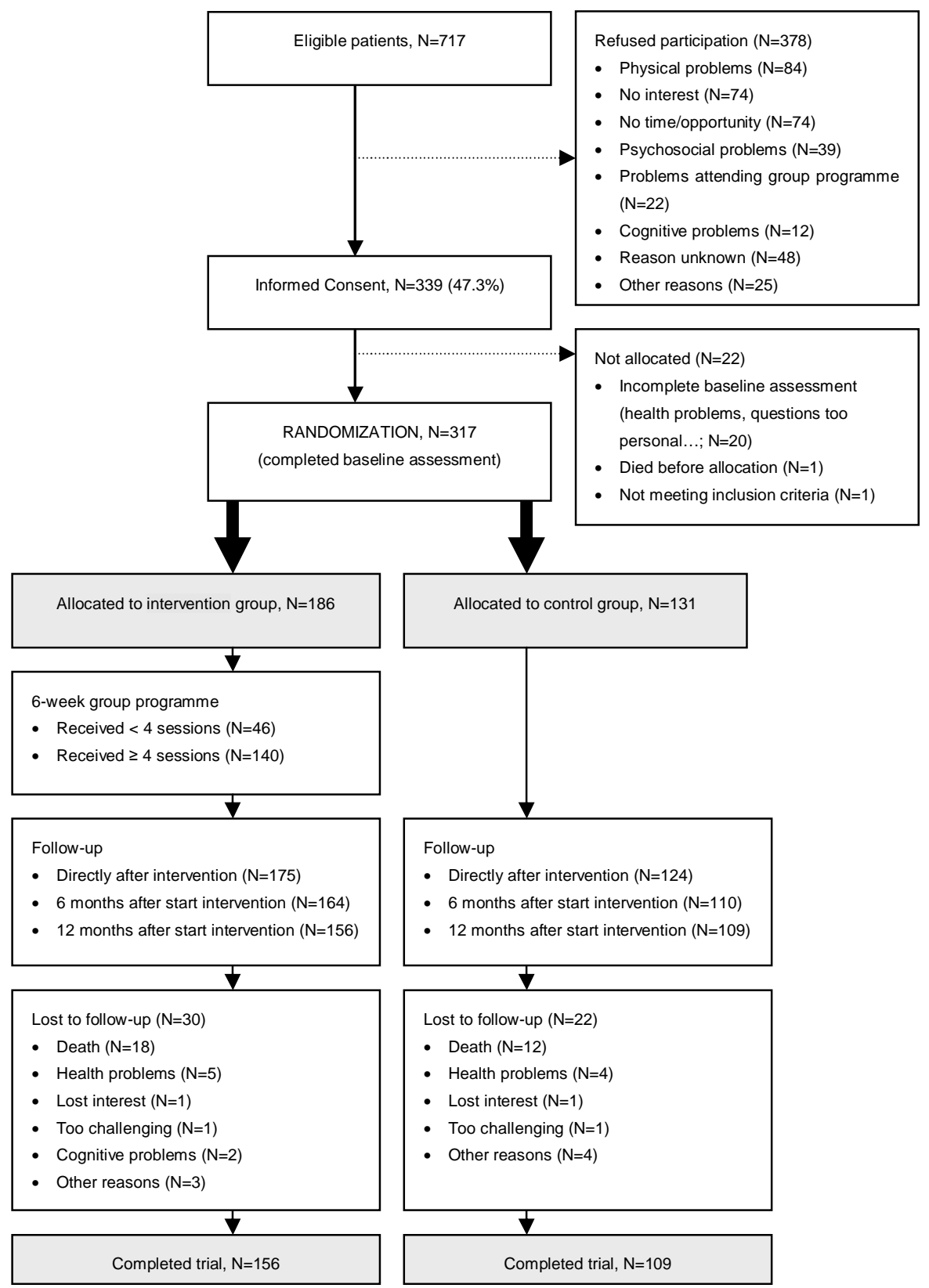

Figure 1: Flow of participants 
Table 1: Background characteristics at baseline $(n=317)$

\begin{tabular}{|c|c|c|c|}
\hline & $\begin{array}{c}\text { Control group } \\
\quad(n=131)\end{array}$ & $\begin{array}{l}\text { Intervention group } \\
\qquad(n=186)\end{array}$ & $P$-value \\
\hline \multicolumn{4}{|l|}{ Background characteristics } \\
\hline Number male (\%) & $89(67.9)$ & $141(75.8)$ & 0.122 \\
\hline Mean age in years at time of inclusion (SD) & $66.8(10.1)$ & $66.6(11.0)$ & 0.882 \\
\hline Number middle educational level $(\%)^{*}$ & $90(68.7)$ & $119(64.3)$ & 0.207 \\
\hline Number unemployed (\%) & $113(86.3)$ & 169 (90.9) & 0.198 \\
\hline Number not living alone (\%) & $89(67.9)$ & $124(66.7)$ & 0.812 \\
\hline Number NYHA Class II CHF (\%) ${ }^{\dagger}$ & $91(69.5)$ & $120(64.5)$ & 0.358 \\
\hline Mean number of active diseases (SD) & $3.0(1.5)$ & $3.0(1.6)$ & 1.00 \\
\hline Mean cognitive status $(0-\underline{41})^{\ddagger}(\mathrm{SD})$ & $32.4(3.1)$ & $32.7(3.3)$ & 0.407 \\
\hline
\end{tabular}

Data were collected in Maastricht between August 2004 and November 2005.

* Middle educational level; attended secondary (vocational) education.

${ }^{\dagger}$ NYHA-Class II CHF; New York Heart Association Class II symptom severity of congestive heart failure.

${ }^{\ddagger}$ Underlined scores indicate most favourable scores.

\section{Outcomes}

Regarding psychosocial attributes, a significant short-term effect of the CDSMP was found for cognitive symptom management (Table 2). No significant effects were found at six and twelve months of follow-up, nor did the CDSMP significantly improve self-efficacy expectancies and perceived control over time. A significant short-term effect of the CDSMP was found for self-care behaviour, but did not extend to six and twelve months after the start of the programme.

Regarding quality of life, a significant positive short-term effect was found for cardiac-specific quality of life, which came close to statistical significance at six months after the start of the programme (Table 3). All (significant) effect sizes were small to medium (Tables 2 and 3 ). The CDSMP did not affect general quality of life, perceived autonomy, and symptoms of anxiety and feelings of depression over time. 
Table 2: Effects of the CDSMP on psychosocial attributes and self-care behaviour $(n=317)$

\begin{tabular}{|c|c|c|c|c|c|c|}
\hline & \multirow{2}{*}{$\begin{array}{c}\begin{array}{c}\text { Control } \\
\text { group }\end{array} \\
\text { Mean (SD) }\end{array}$} & \multirow{2}{*}{$\begin{array}{c}\text { Intervention } \\
\text { group }\end{array}$} & \multicolumn{2}{|c|}{ Adjusted mixed-effects } & \multirow{2}{*}{$\begin{array}{c}\begin{array}{c}P- \\
\text { value }\end{array} \\
P\end{array}$} & \multirow{2}{*}{$\begin{array}{r}\text { Effec } \\
\text { size } \\
d\end{array}$} \\
\hline & & & $\begin{array}{c}\text { Mean } \\
\text { difference }\end{array}$ & (95\% Cl) & & \\
\hline \multicolumn{7}{|l|}{ Psychosocial attributes } \\
\hline \multicolumn{7}{|l|}{$\begin{array}{l}\text { General self-efficacy } \\
(16 \text { to } \underline{80})^{*}\end{array}$} \\
\hline Baseline & $57.4(11.3)$ & $55.2(11.7)$ & - & - & .087 & - \\
\hline Direct follow-up & $57.6(11.8)$ & $57.0(12.3)$ & -1.32 & $(-3.05-.42)$ & .136 & - \\
\hline 6-month follow-up & $58.4(13.0)$ & $56.3(12.5)$ & -.22 & $(-2.10-1.66)$ & .817 & - \\
\hline 12-month follow-up & $58.4(11.6)$ & $57.9(12.9)$ & -1.59 & $-3.59-.40)$ & .116 & - \\
\hline \multicolumn{7}{|l|}{$\begin{array}{l}\text { Cardiac self-efficacy } \\
(0 \text { to } \underline{36})^{*}\end{array}$} \\
\hline Baseline & $35.0(8.1)$ & $34.6(7.8)$ & - & - & .631 & - \\
\hline Direct follow-up & $37.1(7.6)$ & $36.8(7.5)$ & .01 & $(-1.42-1.45)$ & .986 & - \\
\hline 6-month follow-up & $37.3(6.8)$ & $35.6(8.5)$ & 1.13 & $(-.48-2.73)$ & .170 & - \\
\hline 12-month follow-up & $36.4(7.6)$ & $36.9(7.6)$ & -.93 & $(-2.50-.63)$ & .241 & - \\
\hline \multicolumn{7}{|l|}{$\begin{array}{l}\text { Perceived control } \\
(7 \text { to } 35)^{\star}\end{array}$} \\
\hline Baseline & $23.3(5.5)$ & $22.8(5.1)$ & - & - & .448 & - \\
\hline Direct follow-up & $24.2(4.9)$ & $24.2(5.7)$ & -.53 & $(-1.52-.46)$ & .296 & - \\
\hline 6-month follow-up & $24.8(5.3)$ & $23.9(5.8)$ & .41 & $(-.67-1.49)$ & .455 & - \\
\hline 12-month follow-up & $24.4(5.5)$ & $23.9(5.6)$ & .33 & $(-.73-1.39)$ & .544 & - \\
\hline \multicolumn{7}{|l|}{$\operatorname{CSM}^{\dagger}(0 \text { to } \underline{25})^{*}$} \\
\hline Baseline & $7.8(4.5)$ & $7.2(4.7)$ & - & - & .288 & - \\
\hline Direct follow-up & $7.6(4.9)$ & $9.3(5.0)$ & -2.28 & $(-3.23--1.32)$ & .000 & .34 \\
\hline 6-month follow-up & $8.1(5.1)$ & $8.6(5.5)$ & -.84 & $(-1.86-.18)$ & .106 & - \\
\hline 12-month follow-up & $7.7(5.1)$ & $8.3(5.5)$ & -.73 & $(-1.89-.43)$ & .215 & - \\
\hline \multicolumn{7}{|l|}{ Self-care behaviour } \\
\hline \multicolumn{7}{|l|}{$\begin{array}{l}\text { Self-care behaviour } \\
(12 \text { to } \underline{60})^{*}\end{array}$} \\
\hline Baseline & $48.3(6.7)$ & $47.7(6.0)$ & - & - & .412 & - \\
\hline Direct follow-up & $48.7(6.5)$ & $49.8(5.8)$ & -1.47 & $(-2.55--.39)$ & .008 & .18 \\
\hline 6-month follow-up & $48.9(6.5)$ & $48.3(6.5)$ & -.11 & $(-1.28-1.06)$ & .856 & - \\
\hline 12-month follow-up & $49.2(6.6)$ & $49.2(6.3)$ & .94 & $(-2.23-.35)$ & .152 & - \\
\hline
\end{tabular}

Data were collected in Maastricht between August 2004 and January 2007.

* Underlined scores indicate most favourable scores.

${ }^{\dagger}$ CSM: Cognitive symptom management.

The per-protocol analysis $(n=271)$ showed largely comparable results. We found significant short-term effects of the CDSMP on cognitive symptom management (means: 7.6 and 9.8, respectively; mean difference: $-2.90 ; 95 \%$ Cl: $-3.88--1.92 ; p=0.000 ; d=0.45$ ), self-care behaviour (means: 48.7 and 49.9, respectively; mean difference: $-1.47 ; 95 \% \mathrm{Cl}:-2.59--0.34 ; \mathrm{p}=0.011$; $d$ $=0.18$ ) and cardiac-specific quality of life (means: 67.1 and 66.3, respectively; mean difference: $-4.31 ; 95 \% \mathrm{Cl}:-7.24--1.38 ; p=0.004 ; d=0.04)$. The significant effect of the intervention on cognitive symptom management was prolonged at six months (means: 8.1 and 9.1, respectively; mean difference: $0.17 ; 95 \% \mathrm{Cl}:-2.45--0.33 ; \mathrm{p}=0.010 ; d=0.18$ ) and twelve months (means: 
7.7 and 8.7, respectively; mean difference: $-0.96 ; 95 \% \mathrm{Cl}:-2.43--0.01 ; p=$ $0.031 ; d=0.19$ ) of follow-up (not tabulated).

Table 3: Effects of the CDSMP on quality of life $(n=317)$

\begin{tabular}{|c|c|c|c|c|c|c|}
\hline & \multirow{2}{*}{$\begin{array}{c}\begin{array}{c}\text { Control } \\
\text { group }\end{array} \\
\text { Mean (SD) }\end{array}$} & \multirow{2}{*}{$\begin{array}{c}\text { Intervention } \\
\text { group } \\
\text { Mean (SD) }\end{array}$} & \multicolumn{2}{|c|}{ Adjusted mixed-effects } & \multirow{2}{*}{$\begin{array}{c}P \text {-value } \\
P\end{array}$} & \multirow{2}{*}{$\begin{array}{c}\begin{array}{c}\text { Effect } \\
\text { size }\end{array} \\
d\end{array}$} \\
\hline & & & $\begin{array}{c}\text { Mean } \\
\text { difference } \\
\end{array}$ & $(95 \% \mathrm{Cl})$ & & \\
\hline \multicolumn{7}{|l|}{$\overline{\mathrm{G}-\mathrm{QoL}^{\dagger}-\text { physical }}$} \\
\hline \multicolumn{7}{|l|}{$(0-\underline{100})^{*}$} \\
\hline Baseline & $35.7(9.9)$ & $35.2(9.9)$ & - & - & .651 & - \\
\hline Direct follow-up & $36.6(10.6)$ & $37.8(10.6)$ & -1.67 & $(-3.35-.02)$ & .052 & - \\
\hline 6-month follow-up & $37.0(10.2)$ & $37.4(10.5)$ & -1.40 & $(-3.23-.44)$ & .136 & - \\
\hline 12-month follow-up & $38.4(10.0)$ & $37.7(10.2)$ & -.03 & $(-1.95-1.89)$ & .974 & - \\
\hline \multicolumn{7}{|l|}{$\overline{\mathrm{G}-\mathrm{QoL}}{ }^{\dagger}-$ mental } \\
\hline \multicolumn{7}{|l|}{$(0 \text { to } \underline{100})^{*}$} \\
\hline Baseline & $47.2(11.1)$ & $45.2(12.3)$ & - & - & .137 & - \\
\hline Direct follow-up & $48.0(11.0)$ & $47.7(11.4)$ & -.84 & $(-2.83-1.16)$ & .411 & - \\
\hline 6-month follow-up & $49.4(11.4)$ & $46.9(12.0)$ & 1.49 & $(-.59-3.57)$ & .161 & - \\
\hline 12-month follow-up & $48.7(11.3)$ & $47.7(11.7)$ & .09 & $(-2.30-2.48)$ & .939 & - \\
\hline \multicolumn{7}{|l|}{$\begin{array}{l}\mathrm{C}^{-Q \mathrm{LL}^{\dagger}-\text { summary }} \\
\text { score }(0 \text { to } \underline{100})^{*}\end{array}$} \\
\hline Baseline & $65.6(19.2)$ & $59.0(21.7)$ & - & - & .005 & - \\
\hline Direct follow-up & $67.1(19.4)$ & $65.7(21.7)$ & -4.10 & $(-6.95--1.25)$ & .005 & .06 \\
\hline 6-month follow-up & $68.3(21.4)$ & $64.8(22.6)$ & -3.13 & $(-6.29-.02)$ & .052 & - \\
\hline 12-month follow-up & $69.6(20.4)$ & $66.9(22.1)$ & -2.96 & $(-6.68-.75)$ & .118 & - \\
\hline \multicolumn{7}{|l|}{$\begin{array}{l}\text { Perceived autonomy } \\
(0 \text { to } \underline{100})^{*}\end{array}$} \\
\hline Baseline & $49.7(29.1)$ & $48.4(28.5)$ & - & - & .695 & - \\
\hline Direct follow-up & $49.9(27.9)$ & $51.6(25.7)$ & -1.80 & $(-7.79-4.18)$ & .554 & - \\
\hline 6-month follow-up & $52.1(29.4)$ & $51.2(28.1)$ & .47 & $(-5.93-6.87)$ & .885 & - \\
\hline 12-month follow-up & $54.9(28.5)$ & $50.8(26.0)$ & 3.94 & $(-2.20-10.09)$ & .207 & - \\
\hline \multicolumn{7}{|l|}{$\begin{array}{l}\text { Symptoms of anxiety } \\
(\underline{0} \text { to } 21)^{*}\end{array}$} \\
\hline Baseline & $5.9(4.2)$ & $6.7(5.0)$ & - & - & .139 & - \\
\hline Direct follow-up & $5.5(4.3)$ & $5.6(4.6)$ & .26 & $(-.45-.97)$ & .472 & - \\
\hline 6-month follow-up & $5.6(4.5)$ & $6.0(4.8)$ & -.12 & $(-.83-.59)$ & .745 & - \\
\hline 12-month follow-up & $5.2(4.2)$ & $5.9(4.5)$ & -.50 & $(-1.27-.28)$ & .207 & - \\
\hline \multicolumn{7}{|l|}{ Feelings of depression } \\
\hline \multicolumn{7}{|l|}{$(\underline{0} \text { to } 21)^{*}$} \\
\hline Baseline & $5.6(3.9)$ & $6.5(4.5)$ & - & - & .056 & - \\
\hline Direct follow-up & $5.3(4.3)$ & $5.6(4.3)$ & .28 & $(-.94-.38)$ & .406 & - \\
\hline 6-month follow-up & $5.0(4.3)$ & $6.1(4.8)$ & -.24 & $(-.94-.45)$ & .491 & - \\
\hline 12-month follow-up & $4.8(4.5)$ & $5.9(4.6)$ & -.35 & $(-1.16-.47)$ & .403 & - \\
\hline
\end{tabular}

Data were collected in Maastricht between August 2004 and January 2007.

* Underlined scores indicate most favourable scores.

${ }^{\dagger}$ G-QoL/C-QoL: General/Cardiac-specific quality of life. 


\section{Discussion}

This trial showed that the CDSMP significantly improved cognitive symptom management, self-care behaviour and cardiac-specific quality of life of CHF patients in the short term. The programme did not affect perceived control, perceived autonomy, and symptoms of anxiety and feelings of depression. In addition, no effects were observed for patients' self-efficacy expectancies, although self-efficacy is considered one of the key mechanisms of the CDSMP. ${ }^{12}$ Our results seem therefore less favourable than those in previous evaluation studies by Lorig and colleagues. ${ }^{13,17,18}$ However, when we compare our results with more recent studies on the CDSMP we observe a number of studies that also found only moderate or no effects at all, ${ }^{27-33}$ while several other studies showed more favourable results. ${ }^{19-26}$ None of these studies were conducted solely among CHF patients, so a final conclusion on the effectiveness of the CDSMP for this type of patients may not be drawn yet. In addition, in none of these studies the CDSMP was led by pairs of cardiac nurse specialists and peer leaders.

\section{Study limitations}

To the best of our knowledge, this is the first randomized controlled trial on the effectiveness of the CDSMP among CHF patients. We used a follow-up period of twelve months after the start of the programme in order to study long-term effectiveness of the CDSMP among CHF patients. In addition, data were analysed with mixed-effects linear regression models using all available data for each patient. However, the present study has some limitations as well.

First, the target of 274 patients was not completely reached ( $n=265$; 97\%) (Figure 1), which slightly decreases the power of our trial to find longterm effects. It is, however, unlikely that a sample size of 274 patients would have substantially changed the outcomes of our study. Furthermore, we lost 52 patients (16\%) to follow-up. Although this may threaten generalizability of our results, the proportions and reasons for drop-out were similar in the intervention and control groups.

A second limitation is that we used solely self-reported measures for physical functioning instead of more objective measures such as the sixminute walk test. ${ }^{56}$ Outcomes of self-reported measures and more objective measures, however, in general show comparable results among CHF patients with regard to physical functioning. ${ }^{57,58}$ 


\section{Possible explanations}

There may be several explanations for the fact that the effects of the programme were limited and did not last over time. First, regular care for $\mathrm{CHF}$ patients in The Netherlands is at a relatively high level. Nowadays the majority of the Dutch hospitals have their own CHF management programme, often organized as outpatient clinics, where patients visit CHF nurse specialists on a regular basis. ${ }^{59}$ Furthermore, nearly all people in The Netherlands are covered by healthcare insurance and are able to consult their own general practitioner. For chronically ill people healthcare is relatively easily accessible and the quality of this care is often considered good. ${ }^{33}$ The contrast between our intervention and Dutch regular care is therefore possibly too small for the detection of substantial effects. This may also explain the fact that in another Dutch study evaluating the CDSMP, no favourable effects of the CDSMP were observed on self-efficacy, self-management behaviour, or health status in a heterogeneous chronically-ill patient group. ${ }^{33}$

Second, the programme may be too short or not substantial enough to achieve long-term behavioural change among CHF patients. CHF patients are often faced with taking multiple medications and adhering to nonpharmacological management strategies, which is considered rather challenging. ${ }^{6}$ Furthermore, patients need to constantly adapt to their condition as it deteriorates progressively. For these reasons it may be important to increase the time span of the programme, for example, by adding booster group sessions or individual (telephone-based) follow-ups. This assumption is supported by the fact that in another behavioural self-management programme among CHF patients, in which group sessions and motivational phone calls were performed over a longer time span (fifteen weeks), the physical dimension of quality of life was improved in the long term. ${ }^{58}$ This programme, however, did not affect emotional quality of life.$^{58}$ Increasing the time span is also supported by the fact that the feasibility study that was performed alongside our trial revealed that both participants and leaders recommended extending the programme, particularly for issues related to regimen-adherence and end-of-life issues. ${ }^{36}$

\section{Conclusions}

This trial showed that a generic self-management group programme (CDSMP) significantly improved cognitive symptom management, self-care behaviour and cardiac-specific quality of life of CHF patients in the short term. Based on these findings implementation of the CDSMP is not recommended 
among large groups of CHF patients. Additional research is therefore necessary to study whether adaptation of the CDSMP will increase long-term effectiveness of the programme among CHF patients in healthcare contexts that are comparable with the Dutch healthcare system. Adaptations which could be considered are the addition of booster group sessions or individual follow-ups, possibly integrated in regular care, to allow patients to set new goals for behavioural change.

\section{Acknowledgements}

This research project was funded by the Netherlands Heart Foundation (2002B005) and the University Hospital Maastricht (PF 179) in The Netherlands. This study was conducted within CAPHRI School for Public Health and Primary Care of Maastricht University in The Netherlands. We thank N. Steverink, H.A. Elzen, and J.P. Slaets from the University Medical Center Groningen in The Netherlands for their willingness to share Dutch intervention materials. The Centre for Data and Information Management (MEMIC), L. van Hoef and V. Schaffers are acknowledged for their assistance in developing the study materials and in the data collection. Finally, we want to thank all patients and practitioners of the University Hospital Maastricht, Atrium Medical Center Heerlen, VieCuri Medical Center Venlo, Laurentius Hospital Roermond, Maasland Hospital Sittard and Elkerliek Hospital Helmond for their efforts in enabling this study to be conducted. 


\section{References}

1. Mosterd A, Hoes AW. Clinical epidemiology of heart failure. Heart 2007;93(9):1137-46.

2. Thomas S, Rich MW. Epidemiology, pathophysiology, and prognosis of heart failure in the elderly. Clin Geriatr Med 2007;23(1):1-10.

3. van Jaarsveld $\mathrm{CH}$, Ranchor AV, Kempen GI, Coyne JC, van Veldhuisen DJ, Sanderman R. Epidemiology of heart failure in a community-based study of subjects aged $>$ or $=57$ years: incidence and long-term survival. Eur $\mathrm{J}$ Heart Fail 2006;8(1):23-30.

4. van Jaarsveld $\mathrm{CH}$, Sanderman R, Miedema I, Ranchor AV, Kempen GI. Changes in health-related quality of life in older patients with acute myocardial infarction or congestive heart failure: a prospective study. J Am Geriatr Soc 2001;49(8):10528.

5. Yu DS, Lee DT, Kwong AN, Thompson DR, Woo J. Living with chronic heart failure: a review of qualitative studies of older people. J Adv Nurs 2008;61(5):47483.

6. van der Wal $\mathrm{MH}$, Jaarsma $\mathrm{T}$. Adherence in heart failure in the elderly: Problem and possible solutions. Int J Cardiol 2008;125(2):203-8.

7. Riegel B, Carlson B. Facilitators and barriers to heart failure self-care. Patient Educ Couns 2002;46(4):287-95.

8. Konstam V, Moser DK, De Jong MJ. Depression and anxiety in heart failure. $J$ Card Fail 2005;11(6):455-63.

9. Moser DK. Psychosocial factors and their association with clinical outcomes in patients with heart failure: why clinicians do not seem to care. Eur J Cardiovasc Nurs 2002;1(3):183-8.

10. Martensson J, Karlsson JE, Fridlund B. Male patients with congestive heart failure and their conception of the life situation. J Adv Nurs 1997;25(3):579-86.

11. Martensson J, Karlsson JE, Fridlund B. Female patients with congestive heart failure: how they conceive their life situation. J Adv Nurs 1998;28(6):1216-24.

12. Lorig KR, Holman H. Self-management education: history, definition, outcomes, and mechanisms. Ann Behav Med 2003;26(1):1-7.

13. Lorig KR, Sobel DS, Stewart AL, Brown BW, Jr., Bandura A, Ritter P, et al. Evidence suggesting that a chronic disease self-management program can improve health status while reducing hospitalization: a randomized trial. Med Care 1999;37(1):5-14.

14. Bandura A. Self-efficacy: the exercise of control. New York: Freeman; 1997.

15. Sherer M, Maddux JE, Mercandante B, Prentice-Dunn S, Jacobs B, Rogers RW. The self-efficacy scale: construction and validation. Psychol Rep 1982;51:663-71.

16. Kempen GI, Sanderman R, Miedema I, Meyboom-de Jong B, Ormel J. Functional decline after congestive heart failure and acute myocardial infarction and the impact of psychological attributes. A prospective study. Qual Life Res 2000;9(4):439-50.

17. Lorig KR, Ritter P, Stewart AL, Sobel DS, Brown BW, Jr., Bandura A, et al. Chronic disease self-management program: 2-year health status and health care utilization outcomes. Med Care 2001;39(11):1217-23. 
18. Lorig KR, Sobel DS, Ritter PL, Laurent D, Hobbs M. Effect of a self-management program on patients with chronic disease. Eff Clin Pract 2001;4(6):256-62.

19. Fu D, Fu H, McGowan P, Shen YE, Zhu L, Yang H, et al. Implementation and quantitative evaluation of chronic disease self-management programme in Shanghai, China: randomized controlled trial. Bull World Health Organ 2003;81(3):174-82.

20. Lorig KR, Ritter PL, Gonzalez VM. Hispanic chronic disease self-management: a randomized community-based outcome trial. Nurs Res 2003;52(6):361-9.

21. Lorig KR, Ritter PL, Jacquez A. Outcomes of border health Spanish/English chronic disease self-management programs. Diabetes Educ 2005;31(3):401-9.

22. Wright CC, Barlow JH, Turner AP, Bancroft GV. Self-management training for people with chronic disease: an exploratory study. Br J Health Psychol 2003;8(Pt 4):465-76.

23. Barlow JH, Wright CC, Turner AP, Bancroft GV. A 12-month follow-up study of self-management training for people with chronic disease: are changes maintained over time? Br J Health Psychol 2005;10(Pt 4):589-99.

24. Swerissen H, Belfrage J, Weeks A, Jordan L, Walker C, Furler J, et al. A randomised control trial of a self-management program for people with a chronic illness from Vietnamese, Chinese, Italian and Greek backgrounds. Patient Educ Couns 2006;64(1-3):360-8.

25. Siu AM, Chan CC, Poon PK, Chui DY, Chan SC. Evaluation of the chronic disease self-management program in a Chinese population. Patient Educ Couns 2007;65(1):42-50.

26. Kennedy A, Reeves D, Bower P, Lee V, Middleton E, Richardson G, et al. The effectiveness and cost effectiveness of a national lay-led self care support programme for patients with long-term conditions: a pragmatic randomised controlled trial. J Epidemiol Community Health 2007;61(3):254-61.

27. Farrell K, Wicks MN, Martin JC. Chronic disease self-management improved with enhanced self-efficacy. Clin Nurs Res 2004;13(4):289-308.

28. Chan SC, Siu AM, Poon PK, Chan CC. Chronic disease self-management program for Chinese patients: a preliminary multi-baseline study. Int $J$ Rehabil Res 2005;28(4):351-4.

29. Griffiths C, Motlib J, Azad A, Ramsay J, Eldridge S, Feder G, et al. Randomised controlled trial of a lay-led self-management programme for Bangladeshi patients with chronic disease. Br J Gen Pract 2005;55(520):831-7.

30. Goeppinger J, Armstrong B, Schwartz T, Ensley D, Brady TJ. Self-management education for persons with arthritis: Managing comorbidity and eliminating health disparities. Arthritis Rheum 2007;57(6):1081-8.

31. Lorig K, Ritter PL, Plant K. A disease-specific self-help program compared with a generalized chronic disease self-help program for arthritis patients. Arthritis Rheum 2005;53(6):950-7.

32. Kendall E, Catalano T, Kuipers P, Posner N, Buys N, Charker J. Recovery following stroke: the role of self-management education. Soc Sci Med 2007;64(3):735-46.

33. Elzen H, Slaets JP, Snijders TA, Steverink N. Evaluation of the chronic disease self-management program (CDSMP) among chronically ill older people in the Netherlands. Soc Sci Med 2007;64(9):1832-41. 
34. Stewart S, MacIntyre K, Hole DJ, Capewell S, McMurray JJ. More 'malignant' than cancer? Five-year survival following a first admission for heart failure. Eur J Heart Fail 2001;3(3):315-22.

35. Willems DL, Hak A, Visser F, Van der Wal G. Thoughts of patients with advanced heart failure on dying. Palliat Med 2004;18(6):564-72.

36. Smeulders ESTF, Van Haastregt JCM, Janssen-Boyne JJJ, Stoffers HEJH, Van Eijk JTM, Kempen GIJM. Feasibility of a group-based self-management program in congestive heart failure patients. Heart Lung in press.

37. Dumville JC, Hahn S, Miles JN, Torgerson DJ. The use of unequal randomisation ratios in clinical trials: a review. Contemp Clin Trials 2006;27(1):1-12.

38. Smeulders ES, van Haastregt JC, van Hoef EF, van Eijk JT, Kempen GI. Evaluation of a self-management programme for congestive heart failure patients: design of a randomised controlled trial. BMC Health Serv Res 2006;6:91.

39. Swedberg K, Cleland J, Dargie H, Drexler H, Follath F, Komajda M, et al. Guidelines for the diagnosis and treatment of chronic heart failure: executive summary (update 2005): The Task Force for the Diagnosis and Treatment of Chronic Heart Failure of the European Society of Cardiology. Eur Heart $J$ 2005;26(11):1115-40.

40. Lorig K, Holman H, Sobel D, Laurent D, Gonzalez V, Minor M. Living a healthy life with chronic conditions: self-management of heart disease, arthritis, diabetes, asthma, bronchitis, emphysema and others. Boulder, Colorado: Bull Publishing Company; 2000.

41. Sullivan MD, LaCroix AZ, Russo J, Katon WJ. Self-efficacy and self-reported functional status in coronary heart disease: a six-month prospective study. Psychosom Med 1998;60(4):473-8.

42. Pearlin LI, Schooler C. The structure of coping. J Health Soc Behav 1978;19(1):221.

43. Lorig K, Stewart A, Ritter P, Gonzalez V, Laurent D, Lynch J. Outcome measures for health education and other health care interventions. Thousand Oaks, California: Sage Publications, Inc.; 1996.

44. Jaarsma T, Stromberg A, Martensson J, Dracup K. Development and testing of the European Heart Failure Self-Care Behaviour Scale. Eur $J$ Heart Fail 2003;5(3):363-70.

45. Hays RD, Sherbourne CD, Mazel RM. The RAND 36-Item Health Survey 1.0. Health Econ 1993;2:217-227.

46. Green CP, Porter CB, Bresnahan DR, Spertus JA. Development and evaluation of the Kansas City Cardiomyopathy Questionnaire: a new health status measure for heart failure. J Am Coll Cardiol 2000;35(5):1245-55.

47. Zigmond AS, Snaith RP. The hospital anxiety and depression scale. Acta Psychiatr Scand 1983;67:361-70.

48. Brandt J, Spencer M, Folstein M. The Telephone Interview for Cognitive Status. NNBN 1988;1:111-7.

49. Bosscher RJ, Smit JH, Kempen GIM. Algemene competentieverwachtingen bij ouderen: Een onderzoek naar de psychometrische kenmerken van de Algemene Competentieschaal (ALCOS) [Global expectations of self-efficacy in the elderly: An investigation of psychometric characteristics of the General Self-Efficacy Scale]. Nederlands Tijdschrift voor de Psychologie 1997;52(6):239-248. 
50. Kempen GIM. Psychometric properties of GLAS baseline measures: a pilot study (in Dutch). Groningen, The Netherlands: Northern Centre for Healthcare Research; 1992.

51. Kempen GI, Sanderman R, Miedema I, Meyboom-de Jong B, Ormel J. Functional decline after congestive heart failure and acute myocardial infarction and the impact of psychological attributes. A prospective study. Quality of Life Research 2000;9(4):439-50.

52. van der Zee KI, Sanderman $\mathrm{R}$. Het meten van de algemene gezondheidstoestand met de RAND-36, een handleiding. Groningen: Noordelijk Centrum voor Gezondheidsvraagstukken; 1993.

53. Spinhoven P, Ormel J, Sloekers PP, Kempen GI, Speckens AE, Van Hemert AM. A validation study of the Hospital Anxiety and Depression Scale (HADS) in different groups of Dutch subjects. Psychological Medicine 1997;27(2):363-70.

54. Arnold R, Ranchor AV, DeJongste MJ, Koeter GH, Ten Hacken NH, Aalbers R, et al. The relationship between self-efficacy and self-reported physical functioning in chronic obstructive pulmonary disease and chronic heart failure. Behav Med 2005;31(3):107-15.

55. Cohen J. A power primer. Psychol Bull 1992;112(1):155-159.

56. Guyatt $\mathrm{G}$. Use of the six-minute walk test as an outcome measure in clinical trials in chronic heart failure. Heart Fail 1987;3:211-7.

57. Arnold R, Ranchor AV, Koeter GH, de Jongste MJ, Sanderman R. Consequences of chronic obstructive pulmonary disease and chronic heart failure: the relationship between objective and subjective health. Soc Sci Med 2005;61(10):2144-54.

58. Shively M, Kodiath M, Smith TL, Kelly A, Bone P, Fetterly L, et al. Effect of behavioral management on quality of life in mild heart failure: a randomized controlled trial. Patient Educ Couns 2005;58(1):27-34.

59. Jaarsma T, Haaijer-Ruskamp FM, Sturm H, Van Veldhuisen DJ. Management of heart failure in The Netherlands. Eur J Heart Fail 2005;7(3):371-5. 



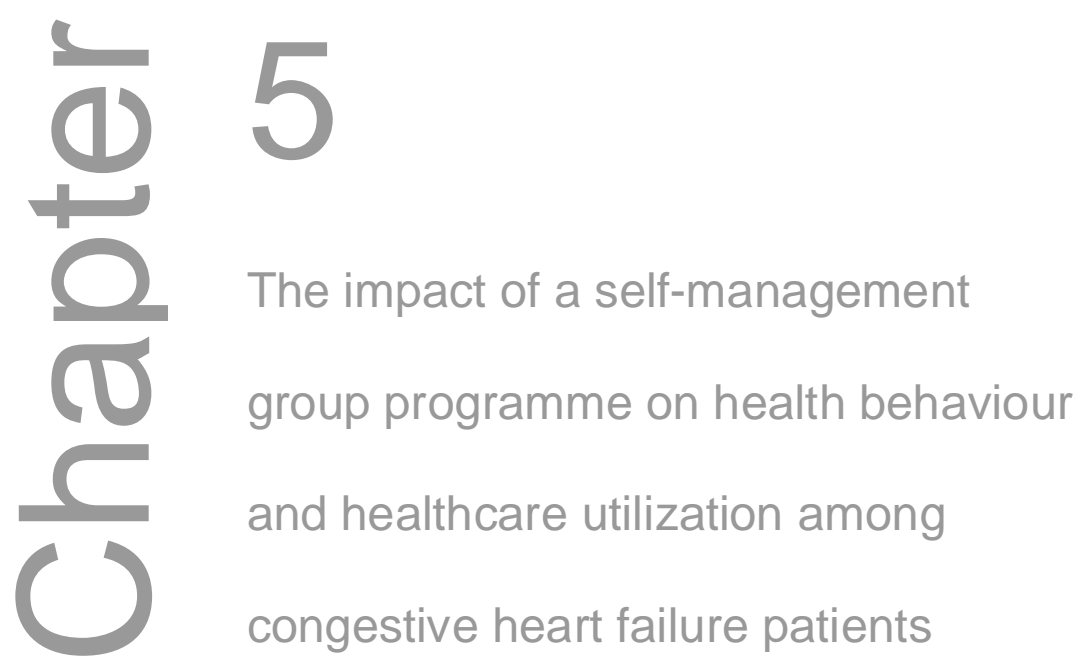

ESTF Smeulders, JCM van Haastregt, T Ambergen, JJJ Janssen-Boyne, JThM van Eijk, GIJM Kempen

European Journal of Heart Failure 2009;11(6):609-616. 


\section{Abstract}

Aims The Chronic Disease Self-Management Programme (CDSMP) emphasizes patients' responsibility for the day-to-day management of their condition(s) and has shown favourable effects on health behaviour and healthcare utilization among various groups of patients with chronic conditions. However, the effects of the CDSMP among congestive heart failure (CHF) patients are unknown. We therefore aimed to assess the effects of the CDSMP on health behaviour and healthcare utilization in patients with CHF.

Methods and results This randomized controlled trial with twelve months of follow-up after start of the programme included $317 \mathrm{CHF}$ patients with a slight to marked limitation of physical activity. Control patients $(n=131)$ received usual care, consisting of regular checkups at an outpatient clinic. Intervention group patients $(n=186)$ received usual care and participated in a six week self-management group programme. Favourable effects on walking for exercise and other physical activities such as aerobic, stretching and strength exercises, sports and gardening were reported in the intervention group immediately after completion of the programme. The effect of the programme on other physical activities extended to six months after the start of the programme. No favourable effects were found for the other outcomes.

Conclusion The CDSMP significantly improved physical activity among CHF patients for up to six months after the end of the programme; however, it did not affect other health behaviour outcomes or healthcare utilization. 


\section{Introduction}

Congestive heart failure (CHF) is a major public health concern, due to aging of the population the prevalence and incidence of CHF is likely to increase dramatically over the coming years, imposing an enormous burden on society in terms of mortality, morbidity, and associated healthcare costs. ${ }^{1}$ Efficient management of $\mathrm{CHF}$ therefore remains a challenge for both patients and healthcare providers. CHF patients face severe symptoms such as fatigue, shortness of breath, and exercise intolerance due to cardiac dysfunction. This substantially affects their physical functioning, quality of life, and mortality rates. ${ }^{2,3}$ Furthermore, patients need to make considerable adjustments to their life style to prevent CHF exacerbations by, for example, adhering to a dietary sodium and fluid restriction, taking prescribed medications and staying physically active. For this reason, patients are encouraged to take responsibility for the day-to-day management of their disease as patients' own self-care abilities are crucial in CHF management. ${ }^{4,5}$ Unfortunately, many patients fail to adhere to the complex treatment regimen. A downward spiral of functional deterioration and non-compliance may then lead to increased healthcare utilization and a progressive decline in the condition of the patient. ${ }^{5-8}$

The Chronic Disease Self-Management Programme (CDSMP) is a generic cognitive-behavioural group programme, which deals with medical, social, and emotional management of a chronic condition. ${ }^{9}$ An important assumption of the programme is that patients can learn to take responsibility for the day-to-day management of their disease(s), ${ }^{9,10}$ which seems crucial for CHF patients. The programme aims to teach patients how to deal with the medical consequences of their disease, to remain socially active in daily life, and to deal with the emotional consequences of being chronically ill. ${ }^{9}$ The CDSMP is based on Bandura's self-efficacy theory. ${ }^{11}$ Self-efficacy refers to the belief that one is capable of performing intended behaviours successfully and is considered a powerful determinant of behavioural change. ${ }^{11,12}$ In his theory, Bandura ${ }^{11}$ recognizes four different sources of self-efficacy: enactive mastery experiences that serve as indicators of people's capability; vicarious experiences that alter efficacy beliefs through comparison with others; social persuasion to strengthen people's beliefs; and physiological and affective states from which people may judge their capabilities. Enhancing self-efficacy expectancies among CHF patients may be very important, as there are indications that low levels of self-efficacy predict functional decline. ${ }^{13}$

Since the 1990s, the CDSMP has been widely disseminated and evaluated within and outside the USA, both in mixed and homogeneous patient groups. ${ }^{10,14-31}$ However, the effectiveness of the programme on health 
behaviour and particularly on healthcare utilization is inconclusive, since some studies showed favourable results, ${ }^{10,14}$ whereas others reported limited ${ }^{15-20}$ or no substantial evidence ${ }^{21-25}$ of effectiveness. These inconclusive findings on health behaviour and healthcare utilization cannot be ascribed to differences in measurement instruments, since all studies used measures comparable to those used by Lorig and colleagues. ${ }^{10}$ Other evaluation studies, however, did not assess healthcare utilization and/or health behaviour at all. ${ }^{26-31}$ Furthermore, the effect of the programme on health behaviour and healthcare utilization among CHF patients, in particular, has not yet been assessed. We therefore conducted a randomized controlled trial to assess the effects of the CDSMP on health behaviour and healthcare utilization in CHF patients with a slight to marked limitation of physical activity.

\section{Methods}

\section{Study design}

Effectiveness of the CDSMP was assessed in a two-group randomized controlled trial with twelve months of follow-up after start of the programme. CHF outpatients were recruited from six hospitals in The Netherlands. Data on health behaviour and healthcare utilization were collected by means of telephone interviews at baseline, directly after the end of the programme and six and twelve months after the start of the programme. The telephone interviews were independently conducted by trained interviewers blinded to group allocation. Twenty-one CDSMP classes were conducted between October 2004 and January 2006 in eleven consecutive cycles, with at least one class and a parallel control group in each cycle. Per cycle and hospital, patients were randomly allocated to the intervention or control group by means of a computerized allocation procedure after completion of the baseline measurement. The randomization procedure was performed by an independent researcher using SPSS 12.0 for Windows. Control patients received usual care, consisting of regular checkups with the cardiologist and/or the CHF nurse specialist at an outpatient clinic. Patients allocated to the intervention group received the self-management group programme in addition to usual care. In each cycle, in which one or more CDSMP classes were planned, the time to recruit patients for the study was limited. As a consequence, equally large, parallel, control groups could not be created, as at least eight patients per cycle were allocated to the intervention group, for reasons of group dynamics. As the group process is very important in the 
CDSMP ${ }^{32}$ at least eight participants are required to participate in the classes, according to Lorig and colleagues. ${ }^{15}$ Therefore, based on the total number of recruited patients per cycle, $59 \%$ of the patients were allocated to the intervention group and $41 \%$ to the control group through the full recruitment period to ensure sufficiently large CDSMP classes. ${ }^{33}$

Based on the sample size calculation, 360 patients needed to be included in the study. The sample size calculation was based on figures on selfefficacy expectancies ${ }^{12}$ (one of the primary outcome variables in the study protocol $)^{34}$ as derived from a Dutch study among newly diagnosed older CHF patients. ${ }^{13}$ To detect a mean difference of at least 4.0 points on self-efficacy expectancies between the two study groups $(S D=11.8$; equivalent to an effect size of 0.34 ), a net number of 274 patients needed to be available for longitudinal analyses to achieve a power of $80 \%$ at an alpha of 0.05 . Approximately 360 patients needed to be included in the study as we assumed a drop-out rate of $25 \%$ during the trial. The trial conformed to the principles outlined in the Declaration of Helsinki ${ }^{35}$ and was approved by the Medical Ethics Committee of Maastricht University/University Hospital Maastricht. $^{34}$

\section{Participants}

CHF outpatients received written information about the study if they (1) had been diagnosed with CHF based on systolic dysfunction (left ventricular ejection fraction (LVEF) < 40\%, NYHA Classes II and III) or diastolic dysfunction (NYHA Classes II and III), as diagnosed by patients' primary cardiologist according to the CHF guidelines. ${ }^{36}$ Patients with diastolic dysfunction were eligible after being admitted at least once to the hospital based on cardiac decompensation after CHF diagnosis. In addition, patients were eligible if they: (2) had been a CHF patient for at least six months at the start of the intervention period, which was considered an appropriate time interval for the CHF diagnosis and therapeutic decisions to have been made; (3) were able to understand, write, and speak Dutch; and (4) were willing to give informed consent to participate in the study. Patients were excluded if they were participating in other studies. Eligible patients underwent a baseline measurement after signing the informed consent form and before randomization. 


\section{Intervention}

The CDSMP is a structured self-management programme and consists of six weekly group sessions of two and a half hours each. The programme incorporates skills mastery, reinterpretation of symptoms, modelling, and social persuasion to enhance self-efficacy expectancies. ${ }^{9,32}$ Skills mastery includes goal-setting and action-planning and takes up about $25-35 \%$ of each session. Patients are supported in action-planning through telephone calls with co-participants. Furthermore, patients are encouraged to set an exercise goal and to make a personal exercise plan during the programme. Reinterpreting symptoms helps to relieve symptom problems such as shortness of breath by, for example, breathing exercises and relaxation techniques. Through action-planning and reinterpreting symptoms the intervention takes into account patients' individual beliefs and cognitions about their illness. Finally, by modelling and social persuasion, participants are expected to become motivated to change their behaviours and beliefs. ${ }^{9}$

At the start of the programme, patients received the CDSMP reference book Living a healthy life with chronic conditions. ${ }^{37}$ The CDSMP classes were led by a cardiac nurse specialist ('professional leader') and a CHF patient ('peer leader'). Cardiac nurse specialists $(n=9)$ participated in the programme to decrease the potential burden on the peer leaders $(n=9)$, to ensure continuity of the programme, and to facilitate implementation of the programme in regular Dutch healthcare. The CDSMP classes were held between October 2004 and January 2006 alongside usual work at the hospital. The peer leaders acted as role models for the other CHF patients in the class. All leaders in the study received training on the CDSMP protocol for four days prior to the intervention period in accordance with CDSMP training policies for programme dissemination (http://patienteducation.stanford.edu/ licensing). The training was provided by one of the authors (E.S.) and a CHF nurse specialist from the University Hospital Maastricht, who had both been instructed as master trainers at Stanford University in March 2004. ${ }^{34}$ Among other things, the leaders learned how to facilitate the process of actionplanning and problem-solving and how to introduce cognitive symptom management techniques to the participants in the classes.

\section{Measurements}

Smoking and drinking behaviour, body mass, and physical activity were used to measure health behaviour. Smoking and drinking behaviour were assessed by asking the patients whether and how much they smoked (cigarettes, cigars 
or pipes) or drank alcoholic beverages at baseline and at each follow up. Based on this we calculated the mean number of cigarettes/cigars/pipes smoked and the mean number of alcoholic drinks per week. Body mass was used as a proxy for nutritional habits and was assessed from the patients weight (in kilograms) and height (in metres). Body Mass Index (BMI) $\left(\mathrm{kg} / \mathrm{m}^{2}\right)$ was then calculated. ${ }^{38}$

Physical activity level was measured using a modified version of the 'Physical Activities Scale' and comprised questions about the frequency of the following three categories: walking for exercise, swimming, and bicycling. ${ }^{39} \mathrm{~A}$ fourth category called 'other physical activities' was added to the scale based upon open-ended questions, in which patients could mention alternative activities. First, from all variously mentioned physical activities walk-related activities were included in the 'walking for exercise' category (e.g. walking the dog and shopping). Exercising on a home trainer was added to the 'bicycling' category. All other mentioned physical activities were then grouped on intensity level. As a result, 'other physical activities' included aerobic, stretching and strength exercises, sports, and gardening, as these were considered to be practised on a moderate level and therefore comparable with walking for exercise, swimming, and bicycling. At each assessment, patients were asked about the total number of minutes they had spent on walking for exercise, swimming, bicycling, and other physical activities in the preceding month.

Healthcare utilization was measured from the self-reported number of contacts with a general practitioner, cardiologist, other medical specialists, or CHF nurse specialist (telephone-based contacts and face-to-face contacts), hospital admissions, days in the hospital, days admitted to a rehabilitation centre, and visits to accident and emergency departments. No distinction was made between CHF- and non-CHF-related hospital admissions, as no valid differentiation between the two types could be made based on patients' selfreports. At baseline, patients were asked about their healthcare utilization in the past six months. At each follow-up assessment, healthcare utilization was measured by asking the patient about healthcare utilization in the preceding months. This resulted in one summary score for each category of healthcare utilization for the total follow-up period. 


\section{Statistical analysis}

Differences in background characteristics and outcomes at baseline between the intervention and control groups were compared by means of Chi-square tests, Mann-Whitney $U$ tests and $t$-tests for independent samples. Differences in the outcome variables were analysed with Mann-Whitney $U$ tests, with the exception of BMI which was analysed with $t$-tests for independent samples. BMI was logarithmically transformed prior to the analyses. Data were analysed according to the intention-to-treat principle. In addition, per-protocol analyses were performed in which intervention group patients were included if they had attended at least four of the six programme sessions. All analyses were based on two-sided tests, and the results were considered significant if $p$ $<0.05$. SPSS for Windows version 12.0.1 was used.

\section{Results}

\section{Participants and baseline characteristics}

A total of 717 patients were eligible for participation in the trial, of whom 339 $(47.3 \%)$ signed the informed consent form and underwent the baseline assessment. The remaining 378 patients refused or were unable to participate in the study, mainly due to physical problems, not being interested, or not having the time or the opportunity to participate in the study (Figure 1).

Baseline characteristics were comparable for the intervention and control groups (Table 1). With regard to the outcome measures, patients in the intervention group tended to drink less alcohol per week and tended to spend less time on other physical activities per month at baseline compared with patients in the control group. However, these differences were not statistically significant (Table 2). 


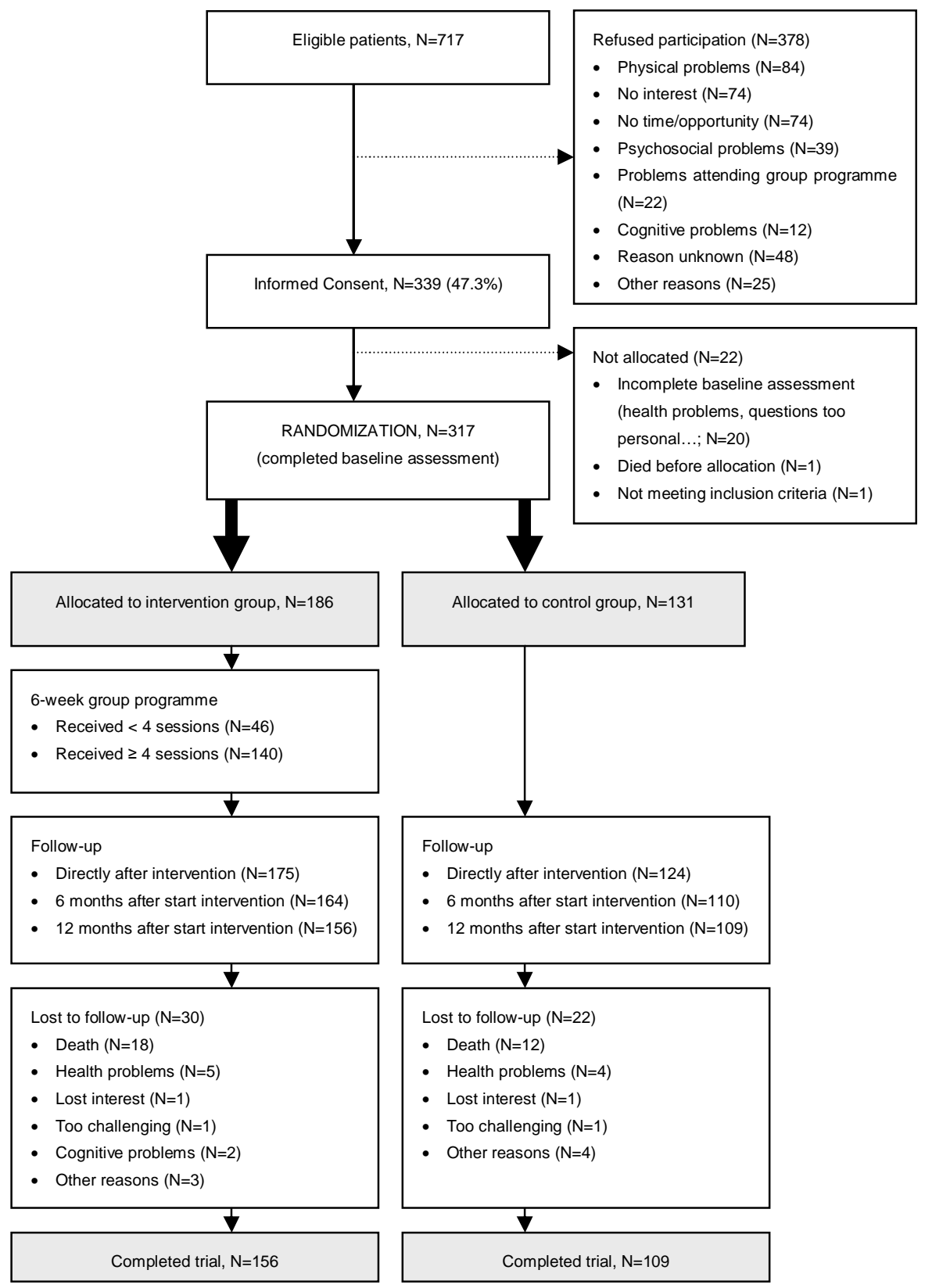

Figure 1: Flow of participants 
Table 1: Patients characteristics at baseline $(n=317)$

\begin{tabular}{|c|c|c|c|}
\hline & $\begin{array}{c}\text { Control group } \\
\quad(n=131)\end{array}$ & $\begin{array}{l}\text { Intervention group } \\
\quad(n=186)\end{array}$ & $P$-value \\
\hline \multicolumn{4}{|l|}{ Background characteristics } \\
\hline Males, $n(\%)$ & $89(67.9)$ & $141(75.8)$ & 0.122 \\
\hline Age in years at time of inclusion, mean (SD) & $66.8(10.1)$ & $66.6(11.0)$ & 0.882 \\
\hline Middle educational level, $n(\%)^{*}$ & $90(68.7)$ & $119(64.3)$ & 0.207 \\
\hline Unemployed, $n(\%)$ & $113(86.3)$ & $169(90.9)$ & 0.198 \\
\hline Not living alone, $n(\%)$ & $89(67.9)$ & $124(66.7)$ & 0.812 \\
\hline NYHA Class II CHF, $n(\%)^{\dagger}$ & $91(69.5)$ & $120(64.5)$ & 0.358 \\
\hline Number of active diseases, mean (SD) ${ }^{13}$ & $3.0(1.5)$ & $3.0(1.6)$ & 1.00 \\
\hline Cognitive status $(0-41)^{\ddagger}$, mean $(S D)^{\S}$ & $32.4(3.1)$ & $32.7(3.3)$ & 0.407 \\
\hline
\end{tabular}

* Middle educational level; attended secondary (vocational) education.

${ }^{\dagger}$ NYHA-Class II CHF; New York Heart Association Class II symptom severity of congestive heart failure.

${ }^{\ddagger}$ Underlined score indicate the most favourable score.

$\S$ Cognitive status as measured with the Telephone Interview for Cognitive Status (TICS).

\section{Outcomes}

Table 2 shows the effects of the CDSMP programme on health behaviour outcomes. No significant effects on smoking or drinking behaviour or body mass were found. Immediately after the end of the programme, patients in the intervention group walked for significantly more minutes per month and spent significantly more minutes per month on other physical activities compared with control patients. The mean time spent on walking increased by 25 minutes per month in the control group and by 200 minutes per month in the intervention group. In terms of other physical activities such as aerobic, stretching and strength exercises, sports, and gardening, the mean time spent on these activities decreased by 52 minutes per month in the control group but increased by 81 minutes per month in the intervention group. The favourable effect on other physical activities extended to six months of followup from the start of the programme (Table 2). No favourable long-term effect on walking for exercise was found. Furthermore, no significant differences between intervention and control group patients were found for swimming or bicycling (Table 2).

Regarding healthcare utilization, no significant differences were found between the control and intervention groups for the number of contacts with a general practitioner during the total follow-up period (both on average 4.6 contacts), contacts with a cardiologist (both on average 2.9 contacts), contacts with other medical specialists (on average 2.6 versus 3.3 contacts, respectively), contacts with a CHF nurse specialist (telephone-based contacts; on average 1.5 versus 2.0 contacts, respectively, and face-to-face contacts; on average 2.1 versus 1.8 contacts, respectively), hospital 
admissions (both on average 0.4 admissions), days in the hospital (on average 3.7 versus 4.9 days, respectively) and visits to accident and emergency departments (both on average 0.6 visits) (data not shown).

Table 2: Effects of the CDSMP on health behaviour $(n=317)$

\begin{tabular}{|c|c|c|c|c|c|}
\hline & \multicolumn{2}{|c|}{$\begin{array}{c}\text { Control group } \\
(n=131)\end{array}$} & \multicolumn{2}{|c|}{$\begin{array}{l}\text { Intervention group } \\
(n=186)\end{array}$} & \multirow{2}{*}{$\begin{array}{c}\begin{array}{c}P- \\
\text { value }\end{array} \\
P\end{array}$} \\
\hline & Mean (SD) & Median & Mean (SD) & Median & \\
\hline \multicolumn{6}{|c|}{ Smoking (cigarettes p/week, $n$ ) } \\
\hline Baseline & $13.5(40.7)$ & 0.0 & $10.2(29.6)$ & 0.0 & 0.587 \\
\hline Direct follow-up & $11.7(37.0)$ & 0.0 & $9.0(26.3)$ & 0.0 & 0.893 \\
\hline 6-month follow-up & $8.4(25.1)$ & 0.0 & $9.6(28.8)$ & 0.0 & 0.902 \\
\hline 12-month follow-up & $9.7(27.9)$ & 0.0 & $10.0(29.8)$ & 0.0 & 0.878 \\
\hline \multicolumn{6}{|c|}{ Drinking (alcoholic drinks p/week, $n$ ) } \\
\hline Baseline & $3.7(6.3)$ & 0.0 & $2.8(5.0)$ & 0.0 & 0.063 \\
\hline Direct follow-up & $3.7(6.2)$ & 0.0 & $3.6(6.9)$ & 0.0 & 0.492 \\
\hline 6-month follow-up & $3.9(6.6)$ & 0.0 & $3.3(6.5)$ & 0.0 & 0.122 \\
\hline 12-month follow-up & $3.7(6.2)$ & 0.0 & $3.2(5.8)$ & 0.0 & 0.639 \\
\hline \multicolumn{6}{|l|}{ Body mass index ${ }^{*}$} \\
\hline Baseline & $26.6(4.1)$ & 26.6 & $27.0(5.0)$ & 26.2 & 0.560 \\
\hline Direct follow-up & $26.6(4.2)$ & 26.5 & $27.0(5.0)$ & 26.1 & 0.549 \\
\hline 6-month follow-up & $27.0(5.4)$ & 26.6 & $27.0(5.3)$ & 26.0 & 0.979 \\
\hline 12-month follow-up & $27.0(4.0)$ & 26.9 & $27.2(5.8)$ & 26.2 & 0.853 \\
\hline \multicolumn{6}{|c|}{ Walking for exercise (min./month) } \\
\hline Baseline & $563.3(713.8)$ & 270.0 & $572.4(791.0)$ & 315.0 & 0.877 \\
\hline Direct follow-up & $588.1(681.7)$ & 360.0 & $772.0(998.0)$ & 540.0 & 0.034 \\
\hline 6-month follow-up & $531.0(780.4)$ & 270.0 & $753.3(1050.1)$ & 450.0 & 0.068 \\
\hline 12-month follow-up & $552.8(706.5)$ & 315.0 & $628.2(762.7)$ & 360.0 & 0.269 \\
\hline \multicolumn{6}{|l|}{ Swimming (min./month) } \\
\hline Baseline & $41.7(181.4)$ & 0.0 & $22.2(67.0)$ & 0.0 & 0.258 \\
\hline Direct follow-up & $30.1(141.8)$ & 0.0 & $33.8(109.6)$ & 0.0 & 0.331 \\
\hline 6-month follow-up & $52.8(205.7)$ & 0.0 & $26.8(86.5)$ & 0.0 & 0.217 \\
\hline 12-month follow-up & $51.1(196.3)$ & 0.0 & $47.8(181.5)$ & 0.0 & 0.991 \\
\hline \multicolumn{6}{|l|}{ Bicycling (min./month) } \\
\hline Baseline & $209.1(423.0)$ & 0.0 & $260.9(526.0)$ & 0.0 & 0.872 \\
\hline Direct follow-up & $220.8(382.8)$ & 0.0 & $302.3(520.4)$ & 40.0 & 0.176 \\
\hline 6-month follow-up & $228.5(471.7)$ & 0.0 & $280.6(604.2)$ & 0.0 & 0.675 \\
\hline 12-month follow-up & $233.5(469.5)$ & 0.0 & $290.7(614.0)$ & 25.0 & 0.356 \\
\hline \multicolumn{6}{|c|}{ Other physical activities (min./month) ${ }^{\dagger}$} \\
\hline Baseline & $146.0(846.2)$ & 0.0 & $144.0(580.5)$ & 0.0 & 0.076 \\
\hline Direct follow-up & $93.7(219.1)$ & 0.0 & $224.5(597.6)$ & 0.0 & 0.006 \\
\hline 6-month follow-up & $86.3(198.0)$ & 0.0 & $205.9(649.5)$ & 0.0 & 0.036 \\
\hline 12-month follow-up & $141.9(309.7)$ & 0.0 & $282.5(937.0)$ & 0.0 & 0.248 \\
\hline
\end{tabular}

* Analysed with $t$-tests for independent samples after logarithmic transformation. Unadjusted mean scores, SDs and median scores are presented.

${ }^{\dagger}$ Other physical activities: aerobic, stretching and strength exercises, sports and gardening. 
Due to the fact that only a few patients had been admitted to a rehabilitation centre at baseline ( $n=4$ in the control group and $n=1$ in the intervention group) and during follow-up ( $n=1$ in the control group and $n=4$ in the intervention group), this outcome had to be excluded from the analyses.

The results of the per-protocol analyses (including control group patients and intervention group patients who attended at least four sessions; $n=271$ ) were similar to the results of the intention-to-treat analyses (data not shown).

\section{Discussion}

This trial shows that the CDSMP substantially improved the time spent on walking for exercise among CHF patients immediately after the end of the programme. In addition, patients in the intervention group spent more time on other physical activities such as aerobic, stretching and strength exercises, sports, and gardening, directly after the programme and at six months of follow-up after the start of the programme. No significant differences were found at twelve months of follow-up. In addition, the programme had no favourable effects on drinking or smoking behaviour, body mass, or healthcare utilization. Therefore, our results seem to be in line with previous studies, showing limited evidence of a beneficial effect of the CDSMP on health behaviour and healthcare utilization. ${ }^{15-20}$

The modest results on health behaviour and healthcare utilization might be explained by the fact that the contrast between the intervention and control group was too small for the detection of substantial additional effects of the programme. In The Netherlands, standard CHF care is at a relatively high level, since most Dutch hospitals including those in our study have a CHF management programme, which often comprises exercise programmes, behavioural interventions, and regular checkups with education and counselling by a multidisciplinary team. ${ }^{40}$ One may consider that attention effects should be controlled for in the control group to make a clear comparison between the two study groups. However, attention through social support and exchanging personal experiences may be considered an essential component of the group intervention that is based on Bandura's selfefficacy theory. ${ }^{9,11}$ A second explanation might be the fact that the patients in the study already had low baseline levels of smoking and drinking, which makes it difficult to detect favourable effects of the programme. Most patients in the study were non-smokers or drank no more than the recommended maximum number of alcoholic beverages. ${ }^{36}$ Finally, the modest results on health behaviour may have been affected by patients' emotional state, as 
emotional and symptom distress are associated with low motivation for behavioural change and physical inactivity. ${ }^{41,42}$ However, exploring the influence of emotional state on motivation for and maintenance of health behaviour change was beyond the scope of the present study.

The favourable short-term results of the programme on physical activity are consistent with the results of a feasibility study that was performed alongside this trial. ${ }^{43}$ This feasibility study showed that the percentage of participants who were still exercising according to their personal exercise plan fell from $78 \%$ directly after the end of the programme to $46 \%$ one year after attending the programme. ${ }^{43}$ Thus, in order to extend the short-term effects on physical activity, it seems important to increase long-term exercise adherence.

Duncan and Pozeh ${ }^{44}$ evaluated the additional value of nurse support on long-term exercise adherence among a small group of older CHF patients ( $n$ $=16$; mean age $=66$ years). Patients in the intervention and control groups underwent a twelve week group exercise programme and were instructed to do aerobic exercises at home; this was followed by a twelve week period of home-based exercise only. During both phases of the programme, exercise goals were set for the home-based exercise. However, patients in the intervention group were supported by a research nurse in terms of individualized graphic feedback and problem-solving techniques to improve exercise adherence. The study showed that exercise adherence was significantly improved during the second phase of the programme (i.e. homebased exercise only) in the patients who received nurse support when compared with control patients. ${ }^{44}$ This study gives an indication that nurse support may be effective in maintaining exercise adherence at home, which might be important in the follow-up period after attending a supervised programme. Motivational interviewing may also be considered an effective tool for enhancing exercise adherence. Brodie and colleagues ${ }^{45,46}$ evaluated an eight week home-based programme which was based on motivational interviewing to explore ambivalence to physical activity and to integrate physical activity into the daily routine of older CHF patients ( $n=92$, of whom 60 completed follow-up; mean age $=78$ years). Patients who attended the programme significantly improved physical activity at five months of follow-up, compared with patients who received standard recommendations regarding physical activity. Unfortunately, the studies of both Duncan and Pozehl ${ }^{44}$ and Brodie and co-workers ${ }^{45,46}$ comprised rather small sample sizes and did not study the long-term effectiveness of the programmes. Nevertheless, these studies give indications that supporting exercise on a daily level may improve exercise adherence among CHF patients. 
Nurse specialists may have an explicit role in supporting long-term exercise adherence, perhaps even more than CHF peers. Although according to the CDSMP, CHF peers may be important in supporting behavioural change and adhering to these new behaviours, maintaining a self-support group consisting only of CHF peers may be challenging. In a feasibility study that was performed alongside this trial, ${ }^{43}$ we found that about $60 \%$ of the participants $(n=111)$ had called co-participants at least once for support, with an average of 2.5 calls during the six week programme (range 1-6). In addition, only a small group $(n=15)$ had kept in contact with one or more co-participants after one year of follow-up. Therefore, we consider nurse support on exercise adherence a better alternative when compared with the creation and maintenance of self-support groups of CHF peers. Enhancing exercise adherence at follow-up (i.e. after patients have attended the CDSMP class) could be included in outpatient CHF care during regular checkups. Nurse support could focus on continuing exercising according to plan and setting new goals to improve the integration of physical activity into patients' daily routines. In addition, nurse specialists may be able to adjust personal exercise goals if the patient's medical condition deteriorates or improves.

The present study has some limitations. First, all measures on health behaviour and healthcare utilization were self-reported. In addition, patients were questioned about their physical activities in the previous month, which may have been influenced by unforeseen circumstances like the weather or acute health problems. However, since both the intervention and control groups were measured in the same time period, it is unlikely that unforeseen fluctuations would have substantially influenced the results. Healthcare utilization was measured retrospectively, which may have resulted in some kind of recall-bias. Medical records were not reviewed to confirm the selfreports given by the patients. Finally, to obtain patients' BMI, height was recorded once at baseline, but weight was recorded at each assessment. A second limitation of the study is that the outcomes may have been affected by sample bias, as only about half of the eligible patients agreed to participate in the study (Figure 1). For example, about one-third of the non-participants $(35.7 \% ; n=135)$ were physically or mentally unable to participate in the study. These patients may have been the most in need of targeting for behavioural change. However, one-third of the randomized patients in our study had NYHA Class III symptoms, indicating that our sample comprised symptomatic CHF patients as well. Therefore, we believe that the influence of sample bias on the basis of perceived health is somewhat limited.

Based on the results of our trial, we conclude that the CDSMP improves physical activity in the short term among CHF patients, but may need 
adaptations to affect healthcare utilization and to improve exercise adherence in the long term, in healthcare settings such as those in The Netherlands. Additional research is necessary to study whether adaptation of the CDSMP will increase effectiveness of the programme on health behaviour and healthcare utilization. In addition, future research should explore the influence of emotional and symptom distress on the motivation for and maintenance of health behaviour change.

\section{Acknowledgements}

We thank the Netherlands Heart Foundation (2002B005) and the University Hospital Maastricht (PF 179) in The Netherlands for funding this research project. The project was conducted within CAPHRI School for Public Health and Primary Care of Maastricht University in The Netherlands in cooperation with Lorig and colleagues from Stanford University. We thank N. Steverink, H.A. Elzen and J.P. Slaets from the University Medical Center Groningen, The Netherlands, for their willingness to share Dutch intervention materials. The Centre for Data and Information Management (MEMIC) of Maastricht University is acknowledged for their assistance in the data collection. We also thank all patients and practitioners of the University Hospital Maastricht, Atrium Medical Center Heerlen, VieCuri Medical Center Venlo, Laurentius Hospital Roermond, Maasland Hospital Sittard, and Elkerliek Hospital Helmond for their efforts that enabled evaluating the CDSMP among CHF patients in The Netherlands. 


\section{References}

1. Thomas S, Rich MW. Epidemiology, pathophysiology, and prognosis of heart failure in the elderly. Clin Geriatr Med 2007;23(1):1-10.

2. van Jaarsveld $\mathrm{CH}$, Ranchor AV, Kempen GI, Coyne JC, van Veldhuisen DJ, Sanderman R. Epidemiology of heart failure in a community-based study of subjects aged $>$ or $=57$ years: incidence and long-term survival. Eur $J$ Heart Fail 2006;8(1):23-30.

3. van Jaarsveld $\mathrm{CH}$, Sanderman R, Miedema I, Ranchor AV, Kempen GI. Changes in health-related quality of life in older patients with acute myocardial infarction or congestive heart failure: a prospective study. J Am Geriatr Soc 2001;49(8):10528.

4. Riegel B. Foreword: Self-care of heart failure: what is the state of the science? $J$ Cardiovasc Nurs 2008;23(3):187-9.

5. Evangelista LS, Shinnick MA. What do we know about adherence and self-care? $J$ Cardiovasc Nurs 2008;23(3):250-7.

6. Riegel B, Carlson B. Facilitators and barriers to heart failure self-care. Patient Educ Couns 2002;46(4):287-95.

7. van der $\mathrm{Wal} \mathrm{MH}$, Jaarsma $\mathrm{T}$. Adherence in heart failure in the elderly: Problem and possible solutions. Int J Cardiol 2008;125(2):203-8.

8. Moser DK, Watkins JF. Conceptualizing self-care in heart failure: a life course model of patient characteristics. J Cardiovasc Nurs 2008;23(3):205-218.

9. Lorig KR, Holman H. Self-management education: history, definition, outcomes, and mechanisms. Ann Behav Med 2003;26(1):1-7.

10. Lorig KR, Sobel DS, Stewart AL, Brown BW, Jr., Bandura A, Ritter P, et al. Evidence suggesting that a chronic disease self-management program can improve health status while reducing hospitalization: a randomized trial. Med Care 1999;37(1):5-14.

11. Bandura A. Self-efficacy: the exercise of control. New York: Freeman; 1997.

12. Sherer M, Maddux JE, Mercandante B, Prentice-Dunn S, Jacobs B, Rogers RW. The self-efficacy scale: construction and validation. Psychol Rep 1982;51:663-71.

13. Kempen GI, Sanderman R, Miedema I, Meyboom-de Jong B, Ormel J. Functional decline after congestive heart failure and acute myocardial infarction and the impact of psychological attributes. A prospective study. Qual Life Res 2000;9(4):439-50.

14. Lorig KR, Ritter PL, Gonzalez VM. Hispanic chronic disease self-management: a randomized community-based outcome trial. Nurs Res 2003;52(6):361-9.

15. Lorig KR, Sobel DS, Ritter PL, Laurent D, Hobbs M. Effect of a self-management program on patients with chronic disease. Eff Clin Pract 2001;4(6):256-62.

16. Fu D, Fu H, McGowan $P$, Shen YE, Zhu L, Yang $H$, et al. Implementation and quantitative evaluation of chronic disease self-management programme in Shanghai, China: randomized controlled trial. Bull World Health Organ 2003;81(3):174-82.

17. Lorig KR, Ritter PL, Jacquez A. Outcomes of border health Spanish/English chronic disease self-management programs. Diabetes Educ 2005;31(3):401-9. 
18. Wright CC, Barlow JH, Turner AP, Bancroft GV. Self-management training for people with chronic disease: an exploratory study. Br J Health Psychol 2003;8(Pt 4):465-76.

19. Barlow JH, Wright CC, Turner AP, Bancroft GV. A 12-month follow-up study of self-management training for people with chronic disease: are changes maintained over time? Br J Health Psychol 2005;10(Pt 4):589-99.

20. Kennedy A, Reeves D, Bower P, Lee V, Middleton E, Richardson G, et al. The effectiveness and cost effectiveness of a national lay-led self care support programme for patients with long-term conditions: a pragmatic randomised controlled trial. J Epidemiol Community Health 2007;61(3):254-61.

21. Lorig K, Ritter PL, Plant K. A disease-specific self-help program compared with a generalized chronic disease self-help program for arthritis patients. Arthritis Rheum 2005;53(6):950-7.

22. Griffiths C, Motlib J, Azad A, Ramsay J, Eldridge S, Feder G, et al. Randomised controlled trial of a lay-led self-management programme for Bangladeshi patients with chronic disease. Br J Gen Pract 2005;55(520):831-7.

23. Elzen $H$, Slaets JP, Snijders TA, Steverink N. Evaluation of the chronic disease self-management program (CDSMP) among chronically ill older people in the Netherlands. Soc Sci Med 2007;64(9):1832-41.

24. Goeppinger J, Armstrong B, Schwartz T, Ensley D, Brady TJ. Self-management education for persons with arthritis: Managing comorbidity and eliminating health disparities. Arthritis Rheum 2007;57(6):1081-8.

25. Elzen H, Slaets JP, Snijders TA, Steverink N. The effect of a self-management intervention on health care utilization in a sample of chronically ill older patients in the Netherlands. J Eval Clin Pract 2008;14(1):159-61.

26. Lorig KR, Ritter P, Stewart AL, Sobel DS, Brown BW, Jr., Bandura A, et al. Chronic disease self-management program: 2-year health status and health care utilization outcomes. Med Care 2001;39(11):1217-23.

27. Farrell K, Wicks MN, Martin JC. Chronic disease self-management improved with enhanced self-efficacy. Clin Nurs Res 2004;13(4):289-308.

28. Chan SC, Siu AM, Poon PK, Chan CC. Chronic disease self-management program for Chinese patients: a preliminary multi-baseline study. Int $J$ Rehabil Res 2005;28(4):351-4.

29. Swerissen H, Belfrage J, Weeks A, Jordan L, Walker C, Furler J, et al. A randomised control trial of a self-management program for people with a chronic illness from Vietnamese, Chinese, Italian and Greek backgrounds. Patient Educ Couns 2006;64(1-3):360-8.

30. Siu AM, Chan CC, Poon PK, Chui DY, Chan SC. Evaluation of the chronic disease self-management program in a Chinese population. Patient Educ Couns 2007;65(1):42-50.

31. Kendall E, Catalano T, Kuipers P, Posner N, Buys N, Charker J. Recovery following stroke: the role of self-management education. Soc Sci Med 2007;64(3):735-46.

32. Lorig K, Gonzalez V, Laurent D. The chronic disease self-management program master trainer's guide. Palo Alto, CA: Stanford University; 1999.

33. Dumville JC, Hahn S, Miles JN, Torgerson DJ. The use of unequal randomisation ratios in clinical trials: a review. Contemp Clin Trials 2006;27(1):1-12. 
34. Smeulders ES, van Haastregt JC, van Hoef EF, van Eijk JT, Kempen GI. Evaluation of a self-management programme for congestive heart failure patients: design of a randomised controlled trial. BMC Health Serv Res 2006;6:91.

35. Rickham PP. Human Experimentation. Code of Ethics of the World Medical Association. Declaration of Helsinki. BMJ 1964;2(5402):177.

36. Swedberg K, Cleland J, Dargie H, Drexler H, Follath F, Komajda M, et al. Guidelines for the diagnosis and treatment of chronic heart failure: executive summary (update 2005): The Task Force for the Diagnosis and Treatment of Chronic Heart Failure of the European Society of Cardiology. Eur Heart $J$ 2005;26(11):1115-40.

37. Lorig K, Holman H, Sobel D, Laurent D, Gonzalez V, Minor M. Living a healthy life with chronic conditions: self-management of heart disease, arthritis, diabetes, asthma, bronchitis, emphysema and others. 2nd ed. Boulder, Colorado: Bull Publishing Company; 2000.

38. Eknoyan G. Adolphe Quetelet (1796-1874)--the average man and indices of obesity. Nephrol Dial Transplant 2008;23(1):47-51.

39. Lorig K, Stewart A, Ritter P, Gonzalez V, Laurent D, Lynch J. Outcome measures for health education and other health care interventions. Thousand Oaks, California: Sage Publications, Inc.; 1996.

40. Jaarsma T, Haaijer-Ruskamp FM, Sturm H, Van Veldhuisen DJ. Management of heart failure in The Netherlands. Eur J Heart Fail 2005;7(3):371-5.

41. Falk K, Patel H, Swedberg K, Ekman I. Fatigue in patients with chronic heart failure - A burden associated with emotional and symptom distress. Eur $J$ Cardiovasc Nurs 2008.

42. Whooley MA, de Jonge $P$, Vittinghoff $E$, Otte $C$, Moos R, Carney RM, et al. Depressive symptoms, health behaviors, and risk of cardiovascular events in patients with coronary heart disease. JAMA 2008;300(20):2379-88.

43. Smeulders ESTF, Van Haastregt JCM, Janssen-Boyne JJJ, Stoffers HEJH, Van Eijk JTM, Kempen GIJM. Feasibility of a group-based self-management program in congestive heart failure patients. Heart Lung in press.

44. Duncan K, Pozehl B. Effects of an exercise adherence intervention on outcomes in patients with heart failure. Rehabil Nurs 2003;28(4):117-22.

45. Brodie DA, Inoue A. Motivational interviewing to promote physical activity for people with chronic heart failure. J Adv Nurs 2005;50(5):518-27.

46. Brodie DA, Inoue A, Shaw DG. Motivational interviewing to change quality of life for people with chronic heart failure: a randomised controlled trial. Int J Nurs Stud 2008;45(4):489-500. 
Effects on health behaviour and healthcare utilization 


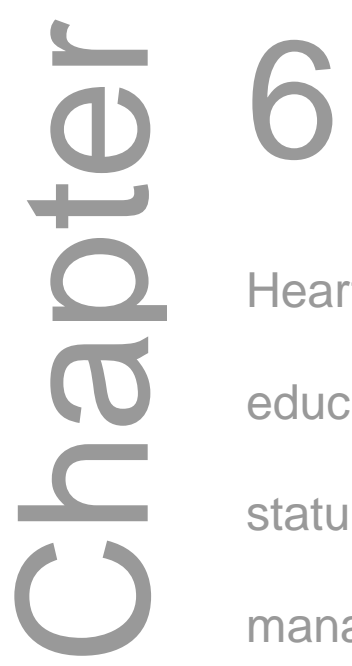

Heart failure patients with a lower

educational level and better cognitive

status benefit most from a self-

management group programme

ESTF Smeulders, JCM van Haastregt, T Ambergen, HEJH Stoffers, JJJ Janssen-Boyne, NHKM Uszko-Lencer, APM Gorgels, CLB Lodewijksvan der Bolt, JThM van Eijk, GIJM Kempen

Submitted 


\section{Abstract}

Introduction The Chronic Disease Self-Management Programme (CDSMP) was recently evaluated among patients with congestive heart failure (CHF) in a randomized controlled trial $(n=317$ ) with twelve months of follow-up after the start of the programme. This trial demonstrated short-term improvements in cardiac-specific quality of life. This study assessed which of the patients participating in this trial benefited most from the CDSMP with respect to cardiac-specific quality of life.

Methods Subgroup analyses were conducted using mixed-effects linear regression models to assess the relationship between patient characteristics and the effects of the CDSMP on cardiac-specific quality of life.

Results In the short term, patients with better cognitive status benefited more from the CDSMP than their poorer functioning counterparts. In addition, lower educated patients benefited more from the CDSMP than their higher educated counterparts during total follow-up.

Discussion and conclusion These results indicate that lower educated patients, in particular, should be encouraged to participate in the CDSMP. In addition, healthcare practitioners are recommended to take into account potential cognitive impairments of patients. Future research should be performed to validate current findings and further explore the conditions under which CHF patients may benefit more from the programme. 


\section{Introduction}

Improving self-management skills among patients with congestive heart failure (CHF) has gained increased attention as it may have favourable effects on symptoms, functional capacity, well being, morbidity, and prognosis. ${ }^{1}$ Healthcare providers encourage CHF patients to increase control over their disease by monitoring symptoms (e.g. daily weighing) and adhering to the treatment regimen (e.g. taking prescribed medications). ${ }^{2-4}$ However, CHF patients often face difficulties in managing the disease in daily life..$^{5-9}$

The Chronic Disease Self-Management Programme (CDSMP) is a generic self-management group programme which emphasizes patients' central role and responsibility in managing their disease. ${ }^{10-13}$ In six weekly two-and-a-half-hour group sessions, patients learn how to deal with the medical, social, and emotional consequences of their chronic condition. ${ }^{10-13}$ Since the 1990s, the CDSMP has been evaluated in several mixed ${ }^{10-12,14-27}$ and homogeneous chronic patient groups ${ }^{28-35}$ worldwide, but not among CHF patients specifically. The CDSMP has showed favourable effects on outcomes such as health behaviour, quality of life, and healthcare utilization. Yet, these favourable results were not consistently found in all studies. ${ }^{15-18,20,28-31,34,35}$ In order to gain insight into the effects of the CDSMP on quality of life (and other outcomes) among CHF patients, Smeulders and colleagues ${ }^{36}$ performed a randomized controlled trial. In addition, programme feasibility among CHF patients was studied. ${ }^{37}$ The CDSMP was found applicable to CHF patients ${ }^{37}$ and improved cardiac-specific quality of life, cognitive symptom management, self-care behaviour (unpublished observations), and physical activity directly after the end of the programme. ${ }^{38}$ However, most of these effects did not endure in the longer term (i.e. six and twelve months after the start of the programme).

The inconsistent findings in previous CDSMP studies as well as the shortterm favourable findings among CHF patients may suggest that the extent to which the programme is effective may partly depend on patient characteristics. Although considerable research has been conducted on the effectiveness of the CDSMP, only some studies explored the differential effects of patient-related factors on programme outcomes. ${ }^{10,20,26,31,33,39}$ In this study, we aim to gain insight into which CHF patients benefited most from the programme in terms of cardiac-specific quality of life. These results may help us to improve the application of the CDSMP among CHF patients. 


\section{Methods}

\section{Study design}

This study is a secondary analysis of the data of a two-group randomized controlled trial $(n=317)$ conducted in six hospitals in The Netherlands. ${ }^{36}$ Data were collected by means of telephone interviews and self-administered questionnaires at baseline, directly after the end of the programme, and six and twelve months after the start of the intervention. The telephone interviews were conducted by trained interviewers blinded to group allocation. Twentyone CDSMP classes (with parallel control groups) were conducted in eleven consecutive cycles between October 2004 and January 2006. After completing the baseline measurement, patients were randomly allocated to the intervention or control group. Control patients received usual care, consisting of regular checkups at an outpatient clinic. Patients allocated to the intervention group attended a self-management group programme (CDSMP) in addition to usual care. ${ }^{36}$ As patient recruitment time was limited in each cycle, equally large parallel control groups could not be created because at least eight patients were allocated to each class of the intervention group, as recommended by Lorig and colleagues. ${ }^{12}$ Therefore, based on the total number of recruited patients per cycle, $59 \%$ of the patients were allocated to the intervention group and $41 \%$ to the control group through the full recruitment period. The sample size calculation was based on figures on selfefficacy expectancies ${ }^{40}$ (one of the primary outcome variables in the study protocol),${ }^{36}$ as derived from a Dutch study among newly-diagnosed older CHF patients. ${ }^{41}$ To detect a mean difference of at least 4.0 points between the two study groups ( $S D=11.8$; equivalent to an effect size of 0.34 ), a net number of 274 patients needed to be available for analyses to achieve a power of $80 \%$ at an alpha of 0.05 . Approximately 360 patients were aimed to be included in the study as we assumed a drop-out rate of $25 \%$ during the trial. The trial was approved by the Medical Ethics Committee of Maastricht University/University Hospital Maastricht. ${ }^{36}$

After the trial period, an expert meeting was organized to discuss which $\mathrm{CHF}$ patients might have benefited most from the CDSMP. Relevant patient characteristics were selected to be included in explorative subgroup analyses. The expert group consisted of four health science researchers, a cardiologist, a general practitioner, and a CHF nurse specialist. The expert group concluded that, based on their experiences, the following patient characteristics were likely to be related to the extent to which persons benefit from the programme: time interval from CHF diagnosis, New York Heart Association (NYHA) classification as an indicator of disease severity, number 
of active comorbid diseases, cognitive status, sex, age, educational level, living arrangements, and job situation. These characteristics were included in the subgroup analyses.

\section{Participants}

As described in detail elsewhere, ${ }^{36}$ patients were eligible for the study if they had been diagnosed with $\mathrm{CHF}$ for at least six months at the start of the intervention period based on systolic or diastolic dysfunction (NYHA Class II to III). Patients with diastolic dysfunction were eligible if they had been admitted at least once to a hospital based on cardiac decompensation after CHF diagnosis. Patients were excluded if they were participating in other studies. Eligible patients received written information about the study and underwent a baseline measurement after signing an informed consent form.

\section{Intervention}

The CDSMP is a generic self-management group programme which addresses daily management problems for patients with chronic conditions. ${ }^{10}$ It emphasizes three self-management tasks to regain control over daily life: (1) to take care of the medical aspects of the disease (medical management); (2) to carry out normal activities to remain socially active (role management); and (3) to manage emotional changes as a consequence of being chronically ill (emotional management). ${ }^{12}$ The CDSMP is based on Bandura's selfefficacy theory ${ }^{42}$ and enhances patients' self-efficacy expectancies by skills mastery, reinterpretation of symptoms, modelling, and social persuasion. ${ }^{10-13}$ Skills mastery includes goal-setting, making weekly action plans, and giving and receiving feedback, which takes up about $25-35 \%$ of each session. ${ }^{43}$ Reinterpreting symptoms deals with modifying people's beliefs about their symptoms in order to relieve symptom problems. The modelling and social persuasion aspects of the group programme aim to support and motivate patients by, for example, peers in changing their behaviours and beliefs. ${ }^{10-13}$ The content of the CDSMP has been described in more detail elsewhere. ${ }^{12,36}$ The CDSMP classes in our study were provided by a trained cardiac nurse specialist and a CHF patient. All leaders (i.e. nine nurse specialists and nine $\mathrm{CHF}$ patients) received four days of training on the CDSMP protocol ${ }^{43}$ before the study. ${ }^{36}$ Patients received the reference book Living a healthy life with chronic conditions ${ }^{44}$ during the first programme session. 


\section{Measurements and analyses}

The main outcome measure was cardiac-specific quality of life, as measured by the Kansas City Cardiomyopathy Questionnaire (KCCQ) (range 0-100; where 100 indicates the most favourable score $)^{45}$ at baseline, directly after the end of the programme, and six and twelve months after the start of the intervention. In addition, disease-related, cognitive, and socio-demographic patient characteristics were measured. Regarding disease-related characteristics, we measured the time interval from CHF diagnosis (in years), NYHA classification (II versus III, corresponding to slight and marked limitation of physical activity respectively), and number of active comorbid diseases. ${ }^{41}$ Cognitive status was measured by the Telephone Interview for Cognitive Status (TICS) (range 0-40; where 40 indicates the most favourable score). ${ }^{46}$ Finally, regarding socio-demographic characteristics, we measured sex, age, educational level (6-point scale; ranging from 'no education or partially attended primary school' to 'university education'), living arrangements ('living alone' versus 'not living alone'), and job situation ('employed' versus 'unemployed'). CHF diagnosis dates and NYHA classifications were obtained from the patients' physicians. All other patient characteristics were collected at baseline by means of a telephone interview. $^{36}$

Prior to the analyses all patient characteristics (except for NYHA classification, sex, living arrangements, and job situation) were recoded into dichotomous variables based on the median score of the total sample $(n=$ $317)$ to form subgroups with lower versus higher scores respectively. Subsequently, patient characteristics and quality of life at baseline were compared between intervention and control groups by means of Chi-square tests, Mann-Whitney $U$ tests, and $t$-tests for independent samples. In addition, differences in cardiac-specific quality of life between intervention and control groups for each subgroup were analysed with mixed-effects linear regression models with an 'unstructured' covariance structure. In the first analyses, we examined subgroup trends over time by analysing between-group differences for each measurement separately in a full mixed-model with four interactions (i.e. group $\times$ time, group $\times$ patient characteristics, time $\times$ patient characteristics and group $\times$ time $\times$ patient characteristics). If no significant three-way interaction was found, subgroup effects were examined in a mixedmodel without a three-way interaction. In this way, subgroup effects at followup could be identified. In all models, we adjusted for baseline scores on cardiac-specific quality of life. Data were analysed according to the intentionto-treat principle based on two-sided tests $(p<0.05)$ using SPSS for Windows version 15.0. 


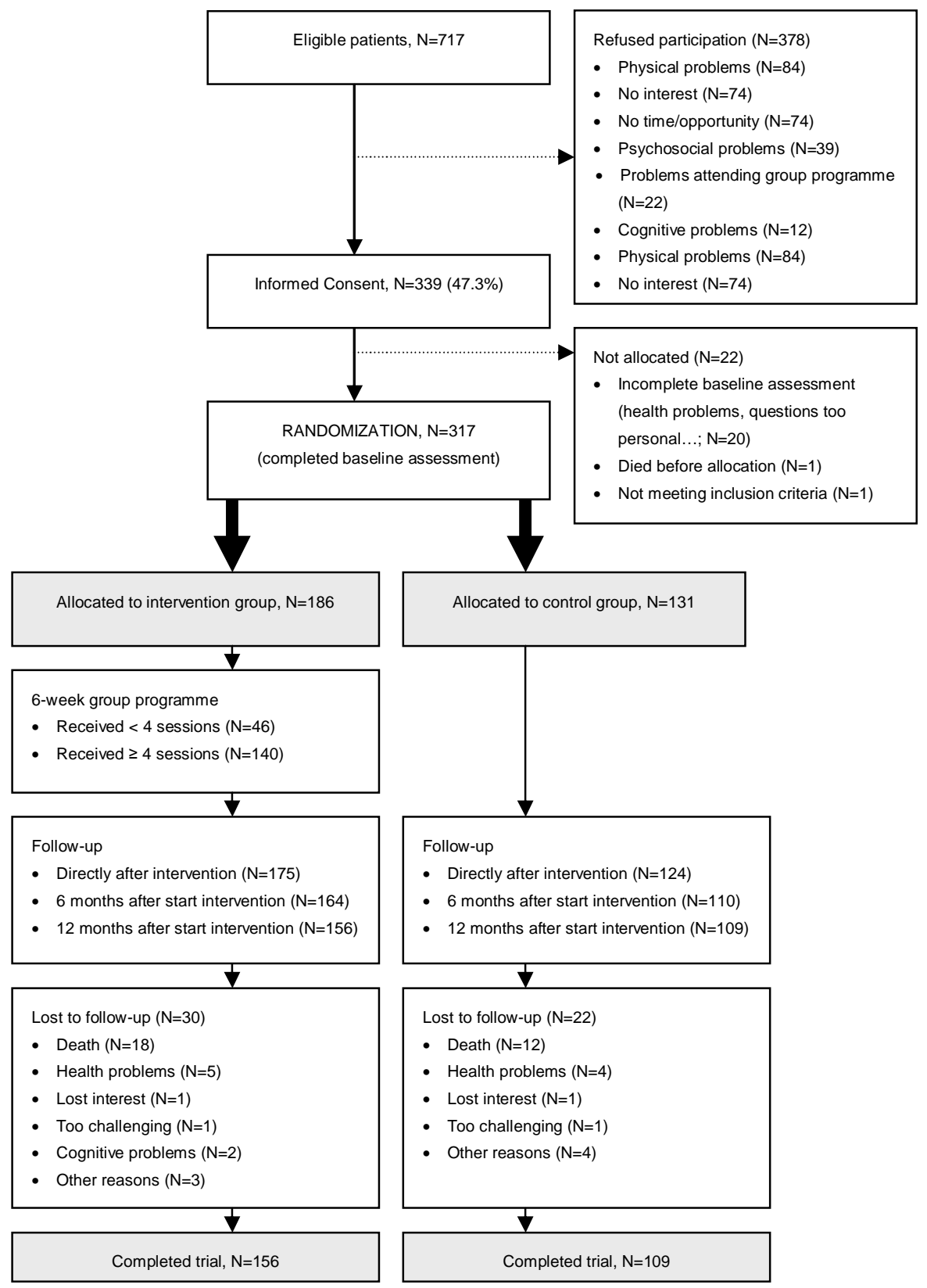

Figure 1: Flow of participants 


\section{Results}

\section{Patient characteristics}

In total, 317 patients participated in the study, of these 186 patients were allocated to the intervention group and 131 patients to the control group. A total of 265 patients $(83.6 \%)$ completed the trial (baseline measurement plus at least the last follow-up measurement). Death was the main reason for 'lost to follow-up' in intervention $(n=18)$ and control groups $(n=12)$ (Figure 1). At baseline, both groups were similar with respect to patient characteristics (Table 1).

Table 1: Patient characteristics at baseline $(n=317)$.

\begin{tabular}{|c|c|c|c|}
\hline & $\begin{array}{l}\text { Intervention group } \\
\qquad(n=186)\end{array}$ & $\begin{array}{c}\text { Control group } \\
\quad(n=131)\end{array}$ & $P$-value \\
\hline \multicolumn{4}{|l|}{ Patient characteristics } \\
\hline Mean time interval from CHF diagnosis (SD)* & $3.2(3.9)$ & $3.2(3.6)$ & 0.883 \\
\hline$\leq 1.9$ years, $n(\%)$ & $95(51.1)$ & $67(51.1)$ & \\
\hline$>1.9$ years, $n(\%)$ & $90(48.4)$ & $64(48.9)$ & \\
\hline Number NYHA Class II CHF (\%) ${ }^{\dagger}$ & $120(64.5)$ & $91(69.5)$ & 0.358 \\
\hline NYHA Class III, $n(\%)$ & $66(35.5)$ & $40(30.5)$ & \\
\hline Mean number of active comorbid diseases (SD) & $3.0(1.6)$ & $3.0(1.5)$ & 1.00 \\
\hline$\leq 3$ active comorbid diseases, $n(\%)$ & $128(68.8)$ & $90(68.7)$ & \\
\hline$>3$ active comorbid diseases, $n(\%)$ & $58(31.2)$ & $41(31.3)$ & \\
\hline Mean cognitive status $(0-\underline{41})(S D)^{\S}$ & $32.7(3.3)$ & $32.4(3.1)$ & 0.407 \\
\hline Score $\leq 33, n(\%)$ & $99(53.2)$ & $78(59.5)$ & \\
\hline Score $>33, n(\%)$ & $87(46.8)$ & $53(40.5)$ & \\
\hline Number male (\%) & $141(75.8)$ & $89(67.9)$ & 0.122 \\
\hline Female, $n(\%)$ & $45(24.2)$ & $42(32.1)$ & \\
\hline Mean age $(S D)^{*}$ & $66.6(11.0)$ & $66.8(10.1)$ & 0.882 \\
\hline$\leq 68.1$ years, $n(\%)$ & $95(51.1)$ & $64(48.9)$ & \\
\hline$>68.1$ years, $n(\%)$ & $91(48.9)$ & $67(51.1)$ & \\
\hline Number low educational level $(\%)^{\ddagger}$ & $108(58.1)$ & $91(69.5)$ & 0.207 \\
\hline Middle/high educational level, $n(\%)$ & $77(41.4)$ & $40(30.5)$ & \\
\hline Number not living alone (\%) & $124(66.7)$ & $89(67.9)$ & 0.812 \\
\hline Alone, $n(\%)$ & $62(33.3)$ & $42(32.1)$ & \\
\hline Number unemployed (\%) & $169(90.9)$ & $113(86.3)$ & 0.198 \\
\hline Employed, $n(\%)$ & $17(9.1)$ & $18(13.7)$ & \\
\hline \multicolumn{4}{|l|}{ Quality of life } \\
\hline Mean cardiac-specific quality of life $(0-100)(S D)^{\S}$ & $59.0(21.7)$ & $65.6(19.2)$ & 0.005 \\
\hline \multicolumn{4}{|c|}{ * Mean time interval from CHF diagnosis was measured at time of inclusion. } \\
\hline \multicolumn{4}{|c|}{${ }^{\dagger}$ NYHA Class II corresponds to slight limitation of physical activity. } \\
\hline \multicolumn{4}{|c|}{$\begin{array}{l}\text { Low educational level; attended primary education/lower secondary (vocational) education ( } \leq 12 \text { years } \\
\text { education), middle/high educational level; attended higher secondary/tertiary (vocational) education ( }>12 \text { yea } \\
\text { of education). }\end{array}$} \\
\hline
\end{tabular}

Time interval from CHF diagnosis in years $($ mean $=3.2 ; \mathrm{SD}=3.8$; median $=$ 1.9 ), number of active comorbid diseases $($ mean $=3.0 ; \mathrm{SD}=1.6$; median $=$ 3.0 ), cognitive status (mean $=32.5 ; \mathrm{SD}=3.2$; median $=33.0)$, age $($ mean $=$ 
66.7; $\mathrm{SD}=10.6 ;$ median $=68.1)$, and educational level $($ mean $=3.3 ; \mathrm{SD}=1.0$; median $=3.0$, corresponding to 'lower secondary (vocational) education') were recoded into dichotomous variables based on the median scores. Job situation was then excluded from the subgroup analyses as only $11 \%$ of the patients in the study $(n=35)$ were employed.

Regarding the main outcome, patients in the intervention group scored significantly lower on cardiac-specific quality of life at baseline than control patients (Table 1). Therefore, the analyses were adjusted for the baseline score on cardiac-specific quality of life.

\section{Subgroup effects on cardiac-specific quality of life}

First, we examined subgroup trends over time. For cognitive status, a significantly different subgroup trend over time was found $(p=0.014)$ (Table 2). Further analyses showed that patients with a better score on cognitive status at baseline benefited more from the programme in the short term than their poorer functioning counterparts. However, no significant differences between the two subgroups according to cognitive status were found at six and twelve months after the start of the programme (Table 2). No significant subgroup trends over time were found for disease-related and sociodemographic patient characteristics (data not shown).

Table 2: Subgroup trend over time for cognitive status as measured in a full mixed-model.

\begin{tabular}{|c|c|c|c|c|c|c|c|}
\hline & & \multirow{2}{*}{$\begin{array}{c}\text { Intervention } \\
\text { group }\end{array}$} & \multirow{2}{*}{$\begin{array}{c}\begin{array}{c}\text { Control } \\
\text { group }\end{array} \\
\begin{array}{c}\text { Adjusted } \\
\text { mean }\end{array}\end{array}$} & \multirow[b]{2}{*}{$\begin{array}{c}\text { Mean } \\
\text { difference }\end{array}$} & \multicolumn{3}{|c|}{ Three-way interaction test ${ }^{\star}$} \\
\hline & & & & & Estimate $^{\ddagger}$ & $P$-value & $(95 \% \mathrm{Cl})$ \\
\hline \multicolumn{8}{|c|}{ Cognitive status } \\
\hline \multicolumn{2}{|c|}{ Full model } & & & & & 0.014 & \\
\hline \multirow[t]{2}{*}{$\mathrm{FU1} 1^{\S}$} & Score $\leq 33$ & 65.80 & 64.78 & 1.02 & -6.324 & 0.027 & $(-11.93--0.72)$ \\
\hline & Score $>33$ & 71.07 & 63.72 & 7.34 & & & \\
\hline \multirow[t]{2}{*}{ FU2 } & Score $\leq 33$ & 66.62 & 63.09 & 3.53 & 1.028 & 0.745 & $(-5.17-7.22)$ \\
\hline & Score $>33$ & 68.21 & 65.71 & 2.50 & & & \\
\hline \multirow[t]{2}{*}{ FU3 } & Score $\leq 33$ & 65.23 & 65.48 & -0.25 & -6.347 & 0.090 & $(-13.69-1.00)$ \\
\hline & Score $>33$ & 70.74 & 64.64 & 6.09 & & & \\
\hline
\end{tabular}

* Three-way interaction (group $\times$ time $\times$ patient characteristics) was tested in a full mixed-effects linear regression model with the covariance structure 'unstructured' while adjusting for the baseline score on cardiacspecific quality of life.

${ }^{\dagger}$ Adjusted mean score on cardiac-specific quality of life per measurement per subgroup.

${ }^{\ddagger}$ Estimate was calculated by subtracting the mean difference of the highest category from the mean difference of the lowest category.

${ }^{\S} \mathrm{FU1}=$ follow-up directly after the end of the programme; FU2 = follow-up at six months after start of the programme; FU3 = follow-up at 12 months after start of the programme. 
Therefore, the subgroup effects at follow-up on disease-related and sociodemographic patient characteristics were assessed by excluding the threeway interaction term from the model. A significant subgroup effect of the programme was found for educational level $(p=0.018)$ in favour of lower educated patients, indicating that lower educated patients benefited more from the programme in terms of cardiac-specific quality of life compared with their higher educated counterparts during the total follow-up period of one year after start of the programme (Table 3). No subgroup effects were found for disease-related characteristics or the other socio-demographic variables (Table 3).

Table 3: Subgroup effects at follow-up as measured in a two-way interactions mixed-model.

\begin{tabular}{|c|c|c|c|c|c|c|}
\hline & \multirow{2}{*}{$\begin{array}{c}\text { Intervention } \\
\text { group } \\
\begin{array}{c}\text { Adjusted } \\
\text { mean }^{\dagger}\end{array} \\
\end{array}$} & \multirow{2}{*}{$\begin{array}{c}\begin{array}{c}\text { Control } \\
\text { group }\end{array} \\
\begin{array}{c}\text { Adjusted } \\
\text { mean }\end{array} \\
\end{array}$} & \multirow[b]{2}{*}{$\begin{array}{c}\text { Mean } \\
\text { difference }\end{array}$} & \multicolumn{3}{|c|}{ Two-way interactions test ${ }^{\star}$} \\
\hline & & & & Estimate $^{\ddagger}$ & $P$-value & $(95 \% \mathrm{Cl})$ \\
\hline \multicolumn{7}{|l|}{ Time interval from } \\
\hline \multicolumn{7}{|l|}{ CHF diagnosis } \\
\hline$\leq 1.9$ years & 68.42 & 64.77 & 3.64 & 0.873 & 0.732 & $(-4.14-5.89)$ \\
\hline$>1.9$ years & 67.11 & 64.34 & 2.77 & & & \\
\hline \multicolumn{7}{|l|}{ NYHA classification } \\
\hline NYHA Class II & 69.06 & 66.10 & 2.97 & -1.489 & 0.585 & $(-6.85-3.87)$ \\
\hline NYHA Class III & 65.51 & 61.05 & 4.46 & & & \\
\hline \multicolumn{7}{|l|}{$\begin{array}{l}\text { Active comorbid } \\
\text { diseases }\end{array}$} \\
\hline$\leq 3$ diseases & 68.86 & 65.13 & 3.73 & 1.611 & 0.563 & $(-3.86-7.08)$ \\
\hline$>3$ diseases & 65.50 & 63.38 & 2.12 & & & \\
\hline Cognitive status ${ }^{\S}$ & - & - & - & - & - & - \\
\hline \multicolumn{7}{|l|}{ Sex } \\
\hline Male & 68.13 & 64.55 & 3.58 & 0.728 & 0.795 & $(-4.78-6.24)$ \\
\hline Female & 67.34 & 64.49 & 2.85 & & & \\
\hline \multicolumn{7}{|l|}{ Age } \\
\hline$\leq 68.1$ years & 69.10 & 66.97 & 2.12 & -2.574 & 0.308 & $(-7.54-2.39)$ \\
\hline$>68.1$ years & 66.83 & 62.14 & 4.70 & & & \\
\hline \multicolumn{7}{|l|}{ Educational level } \\
\hline Low level & 67.68 & 62.39 & 5.29 & 6.321 & 0.018 & $(1.11-11.53)$ \\
\hline Middle/high level & 68.38 & 69.42 & -1.04 & & & \\
\hline \multicolumn{7}{|l|}{ Living arrangements } \\
\hline Alone & 66.87 & 64.77 & 2.10 & -1.913 & 0.485 & $(-7.29-3.47)$ \\
\hline Not alone & 68.45 & 64.43 & 4.01 & & & \\
\hline \multicolumn{7}{|c|}{$\begin{array}{l}\text { * Two-way interactions (i.e. group } \times \text { time, group } \times \text { patient characteristic, time } \times \text { patient characteristic) were } \\
\text { tested in a mixed-effects linear regression model with the covariance structure 'unstructured' while adjusting for } \\
\text { the baseline score on cardiac-specific quality of life. } \\
{ }^{\dagger} \text { Overall adjusted mean score on cardiac-specific quality of life at follow-up per subgroup. } \\
\text { ‡ Estimate was calculated by subtracting the mean difference of the highest category from the mean difference } \\
\text { of the lowest category. } \\
\S \text { Cognitive status was excluded from the analysis as a significantly different subgroup trend over time was } \\
\text { found in the full mixed-model (including 'group } \times \text { time } \times \text { patient characteristic' interaction). }\end{array}$} \\
\hline
\end{tabular}




\section{Discussion and conclusion}

\section{Discussion}

In this study, we explored which CHF patients benefited most from a selfmanagement programme in terms of cardiac-specific quality of life. Multilevel analyses showed that patients with better cognitive functioning benefited significantly more from the CDSMP in the short term than their poorer functioning counterparts. In the longer term (i.e. at six and twelve months after the start of the programme), however, no significant difference in cardiacspecific quality of life was found between the two subgroups, according to cognitive status. In addition, patients who attended at most lower secondary (vocational) education benefited significantly more from the CDSMP than their higher educated counterparts during the total follow-up period.

To our knowledge, this is the first study on the CDSMP which showed evidence for cognitive status and educational level as significant predictors of the effectiveness of the CDSMP on cardiac-specific quality of life. Previous studies on the CDSMP found indications that programme effectiveness varied according to cultural group, age (in favour of patients < 40 years) and baseline levels of self-efficacy and quality of life (in favour of poorer functioning patients). ${ }^{26,39}$ In the other studies, no differential effects were found. ${ }^{10,20,31,33}$ Educational level was also included in the analyses by Reeves and colleagues, ${ }^{39}$ though no differential effect with respect to educational level was found. To our knowledge, cognitive status has not been previously assessed as a potential predictor for CDSMP outcomes.

Our findings suggest that the programme's effect on cardiac-specific quality of life among CHF patients with better cognitive status is limited to the short term, while the programme's positive effect among lower educated CHF patients is more stable over time. Our finding that patients with better cognitive functioning benefited more from the programme in the short term may be explained by the fact that the CDSMP is a cognitive-behavioural intervention which demands specific cognitive skills from the patients. In addition, these cognitive demands may be most urgent while attending the CDSMP programme, which may explain the short-term effect found for cognitive status. The finding that lower educated persons benefited more from the programme may be explained by the fact that lower educated persons have less self-management skills than their higher educated counterparts, so for them there may be more to learn with respect to, for example, diseasespecific knowledge and healthy lifestyles. ${ }^{8,47-53}$

By interpreting the findings of this study, the following limitation needs to be taken into account. In spite of the fact that patients were allocated at 
random to intervention and control groups, patients in the intervention group reported significantly lower cardiac-specific quality of life at baseline than control patients (Table 1). Therefore, patients in the intervention group possibly had more to gain from the programme based on these lower baseline levels of cardiac-specific quality of life. Consequently, we adjusted for the baseline level of cardiac-specific quality of life in all analyses to minimise potential bias of this significant baseline difference.

Future research is necessary to perform pre-planned subgroup analyses to confirm the influence of cognitive status and educational level on the effectiveness of the CDSMP, also in other patient groups. In addition, future research may further explore the conditions under which higher educated $\mathrm{CHF}$ patients may benefit more from self-management programmes.

\section{Conclusion}

Subgroup analyses showed that patients with better cognitive status and lower educational levels benefit most from a group-based self-management programme (CDSMP) in terms of cardiac-specific quality of life. The subgroup effect for cognitive status was only significant in the short term and did not endure in the long term. The subgroup effect for educational level was stable over the total follow-up period of one year after start of the programme.

\section{Practice implications}

These results have implications for healthcare professionals of CHF patients. First, healthcare professionals may encourage patients with a lower educational level, in particular, to participate in CDSMP classes, as these patients benefit most from the programme in terms of cardiac-specific quality of life. However, recruiting and retaining lower educated patients in selfmanagement programmes may be challenging. ${ }^{54,55}$

Second, we recommend healthcare professionals who intend using the CDSMP to take into account potential cognitive impairments of $\mathrm{CHF}$ patients in order to improve the effectiveness of the cognitive-behavioural programme. Cognitive impairments such as memory and attention deficits and difficulties in learning and problem-solving are common among CHF patients. ${ }^{56}$ If the CDSMP is applied to CHF patients, adjusting the programme to ten one-anda-half-hour sessions may, therefore, be considered. ${ }^{37}$ Patients with severe cognitive impairments may be excluded from the programme. 
Finally, based on these findings, screening patients for other patient characteristics than cognitive status and educational level is not recommended, as no differential effects were found on, for example, time interval from CHF diagnosis or NYHA classification. However, as shown in the feasibility study conducted alongside the trial, ${ }^{37}$ twelve patients (10\%) reported that they would have preferred attending the programme earlier after diagnosis or were already familiar with most of the content by the time they attended the programme.

\section{Acknowledgements}

We thank the Netherlands Heart Foundation (2002B005) and the University Hospital Maastricht (PF 179) in The Netherlands for funding this research project. The project was conducted within CAPHRI School for Public Health and Primary Care of Maastricht University in The Netherlands. We thank N. Steverink, H.A. Elzen, and J.P. Slaets from the University Medical Center Groningen in The Netherlands for their willingness to share Dutch intervention materials. The Centre for Data and Information Management (MEMIC) of Maastricht University is acknowledged for their assistance in the data collection. Finally, we want to thank all patients and practitioners of the University Hospital Maastricht, Atrium Medical Center Heerlen, VieCuri Medical Center Venlo, Laurentius Hospital Roermond, Maasland Hospital Sittard, and Elkerliek Hospital Helmond for their efforts that enabled evaluating the CDSMP among CHF patients in The Netherlands. 


\section{References}

1. Dickstein K, Cohen-Solal A, Filippatos G, McMurray JJ, Ponikowski P, PooleWilson PA, et al. ESC Guidelines for the diagnosis and treatment of acute and chronic heart failure 2008 The Task Force for the Diagnosis and Treatment of Acute and Chronic Heart Failure 2008 of the European Society of Cardiology. Developed in collaboration with the Heart Failure Association of the ESC (HFA) and endorsed by the European Society of Intensive Care Medicine (ESICM). Eur J Heart Fail 2008.

2. Riegel B, Dickson VV. A situation-specific theory of heart failure self-care. $J$ Cardiovasc Nurs 2008;23(3):190-6.

3. Evangelista LS, Shinnick MA. What do we know about adherence and self-care? $J$ Cardiovasc Nurs 2008;23(3):250-7.

4. Riegel B. Foreword: Self-care of heart failure: what is the state of the science? $J$ Cardiovasc Nurs 2008;23(3):187-9.

5. Konstam V, Moser DK, De Jong MJ. Depression and anxiety in heart failure. $J$ Card Fail 2005;11(6):455-63.

6. Moser DK. Psychosocial factors and their association with clinical outcomes in patients with heart failure: why clinicians do not seem to care. Eur J Cardiovasc Nurs 2002;1(3):183-8.

7. Riegel B, Carlson B. Facilitators and barriers to heart failure self-care. Patient Educ Couns 2002;46(4):287-95.

8. Rockwell JM, Riegel B. Predictors of self-care in persons with heart failure. Heart Lung 2001;30(1):18-25.

9. Wu JR, Moser DK, Lennie TA, Burkhart PV. Medication adherence in patients who have heart failure: a review of the literature. Nursing Clinics of North America 2008;43(1):133-53.

10. Lorig KR, Sobel DS, Stewart AL, Brown BW, Jr., Bandura A, Ritter P, et al. Evidence suggesting that a chronic disease self-management program can improve health status while reducing hospitalization: a randomized trial. Med Care 1999;37(1):5-14.

11. Lorig KR, Ritter P, Stewart AL, Sobel DS, Brown BW, Jr., Bandura A, et al. Chronic disease self-management program: 2-year health status and health care utilization outcomes. Med Care 2001;39(11):1217-23.

12. Lorig KR, Sobel DS, Ritter PL, Laurent D, Hobbs M. Effect of a self-management program on patients with chronic disease. Eff Clin Pract 2001;4(6):256-62.

13. Lorig KR, Holman $\mathrm{H}$. Self-management education: history, definition, outcomes, and mechanisms. Ann Behav Med 2003;26(1):1-7.

14. Barlow JH, Wright CC, Turner AP, Bancroft GV. A 12-month follow-up study of self-management training for people with chronic disease: are changes maintained over time? Br J Health Psychol 2005;10(Pt 4):589-99.

15. Chan SC, Siu AM, Poon PK, Chan CC. Chronic disease self-management program for Chinese patients: a preliminary multi-baseline study. Int $J$ Rehabil Res 2005;28(4):351-4.

16. Elzen H, Slaets JP, Snijders TA, Steverink N. Evaluation of the chronic disease self-management program (CDSMP) among chronically ill older people in the Netherlands. Soc Sci Med 2007;64(9):1832-41. 
17. Elzen H, Slaets JP, Snijders TA, Steverink N. The effect of a self-management intervention on health care utilization in a sample of chronically ill older patients in the Netherlands. J Eval Clin Pract 2008;14(1):159-61.

18. Farrell K, Wicks MN, Martin JC. Chronic disease self-management improved with enhanced self-efficacy. Clin Nurs Res 2004;13(4):289-308.

19. Fu D, Fu H, McGowan $P$, Shen YE, Zhu L, Yang $H$, et al. Implementation and quantitative evaluation of chronic disease self-management programme in Shanghai, China: randomized controlled trial. Bull World Health Organ 2003;81(3):174-82.

20. Griffiths C, Motlib J, Azad A, Ramsay J, Eldridge S, Feder G, et al. Randomised controlled trial of a lay-led self-management programme for Bangladeshi patients with chronic disease. Br J Gen Pract 2005;55(520):831-7.

21. Kennedy A, Reeves D, Bower P, Lee V, Middleton E, Richardson G, et al. The effectiveness and cost effectiveness of a national lay-led self care support programme for patients with long-term conditions: a pragmatic randomised controlled trial. J Epidemiol Community Health 2007;61(3):254-61.

22. Lorig KR, Ritter PL, Gonzalez VM. Hispanic chronic disease self-management: a randomized community-based outcome trial. Nurs Res 2003;52(6):361-9.

23. Lorig KR, Ritter PL, Jacquez A. Outcomes of border health Spanish/English chronic disease self-management programs. Diabetes Educ 2005;31(3):401-9.

24. Richardson G, Kennedy A, Reeves D, Bower P, Lee V, Middleton E, et al. Cost effectiveness of the Expert Patients Programme (EPP) for patients with chronic conditions. J Epidemiol Community Health 2008;62(4):361-7.

25. Siu AM, Chan CC, Poon PK, Chui DY, Chan SC. Evaluation of the chronic disease self-management program in a Chinese population. Patient Educ Couns 2007;65(1):42-50.

26. Swerissen H, Belfrage J, Weeks A, Jordan L, Walker C, Furler J, et al. A randomised control trial of a self-management program for people with a chronic illness from Vietnamese, Chinese, Italian and Greek backgrounds. Patient Educ Couns 2006;64(1-3):360-8.

27. Wright CC, Barlow JH, Turner AP, Bancroft GV. Self-management training for people with chronic disease: an exploratory study. Br J Health Psychol 2003;8(Pt 4):465-76.

28. Goeppinger J, Armstrong B, Schwartz T, Ensley D, Brady TJ. Self-management education for persons with arthritis: Managing comorbidity and eliminating health disparities. Arthritis Rheum 2007;57(6):1081-8.

29. Kendall E, Catalano T, Kuipers P, Posner N, Buys N, Charker J. Recovery following stroke: the role of self-management education. Soc Sci Med 2007;64(3):735-46.

30. Lorig K, Ritter PL, Plant K. A disease-specific self-help program compared with a generalized chronic disease self-help program for arthritis patients. Arthritis Rheum 2005;53(6):950-7.

31. Barlow JH, Turner AP, Gilchrist M. A randomised controlled trial of lay-led selfmanagement for Myocardial Infarction patients who have completed cardiac rehabilitation. Eur J Cardiovasc Nurs 2009. 
32. McGillion MH, Watt-Watson J, Stevens B, Lefort SM, Coyte P, Graham A. Randomized controlled trial of a psychoeducation program for the selfmanagement of chronic cardiac pain. J Pain Symptom Manage 2008;36(2):12640.

33. Gitlin LN, Chernett NL, Harris LF, Palmer D, Hopkins P, Dennis MP. Harvest health: translation of the chronic disease self-management program for older African Americans in a senior setting. Gerontologist 2008;48(5):698-705.

34. Ludman EJ, Simon GE, Grothaus LC, Luce C, Markley DK, Schaefer J. A pilot study of telephone care management and structured disease self-management groups for chronic depression. Psychiatr Serv 2007;58(8):1065-72.

35. Haas M, Groupp E, Muench J, Kraemer D, Brummel-Smith K, Sharma R, et al. Chronic disease self-management program for low back pain in the elderly. $J$ Manipulative Physiol Ther 2005;28(4):228-37.

36. Smeulders ES, van Haastregt JC, van Hoef EF, van Eijk JT, Kempen GI. Evaluation of a self-management programme for congestive heart failure patients: design of a randomised controlled trial. BMC Health Serv Res 2006;6:91.

37. Smeulders ESTF, Van Haastregt JCM, Janssen-Boyne JJJ, Stoffers HEJH, Van Eijk JTM, Kempen GIJM. Feasibility of a group-based self-management program in congestive heart failure patients. Heart Lung in press.

38. Smeulders ESTF, van Haastregt JCM, Ambergen T, Janssen-Boyne JJJ, van Eijk JTM, Kempen GIJM. The impact of a self-management group programme on health behaviour and healthcare utilisation among congestive heart failure patients. Eur J Heart Fail 2009;11(6):609-16.

39. Reeves D, Kennedy A, Fullwood C, Bower P, Gardner C, Gately C, et al. Predicting who will benefit from an Expert Patients Programme self-management course. Br J Gen Pract 2008;58(548):198-203.

40. Sherer M, Maddux JE, Mercandante B, Prentice-Dunn S, Jacobs B, Rogers RW. The self-efficacy scale: construction and validation. Psychol Rep 1982;51:663-71.

41. Kempen GI, Sanderman R, Miedema I, Meyboom-de Jong B, Ormel J. Functional decline after congestive heart failure and acute myocardial infarction and the impact of psychological attributes. A prospective study. Qual Life Res 2000;9(4):439-50.

42. Bandura A. Self-efficacy: the exercise of control. New York: Freeman; 1997.

43. Lorig K, Gonzalez V, Laurent D. The chronic disease self-management program master trainer's guide. Palo Alto, CA: Stanford University; 1999.

44. Lorig K, Holman H, Sobel D, Laurent D, Gonzalez V, Minor M. Living a healthy life with chronic conditions: self-management of heart disease, arthritis, diabetes, asthma, bronchitis, emphysema and others. 2nd ed. Boulder, Colorado: Bull Publishing Company; 2000.

45. Green CP, Porter CB, Bresnahan DR, Spertus JA. Development and evaluation of the Kansas City Cardiomyopathy Questionnaire: a new health status measure for heart failure. J Am Coll Cardiol 2000;35(5):1245-55.

46. Brandt J, Spencer M, Folstein M. The Telephone Interview for Cognitive Status. NNBN 1988;1:111-7.

47. Philbin EF, Dec GW, Jenkins PL, DiSalvo TG. Socioeconomic status as an independent risk factor for hospital readmission for heart failure. Am J Cardiol 2001;87(12):1367-71. 
48. Rathore SS, Masoudi FA, Wang Y, Curtis JP, Foody JM, Havranek EP, et al. Socioeconomic status, treatment, and outcomes among elderly patients hospitalized with heart failure: findings from the National Heart Failure Project. Am Heart J 2006;152(2):371-8.

49. Sui X, Gheorghiade M, Zannad F, Young JB, Ahmed A. A propensity matched study of the association of education and outcomes in chronic heart failure. Int $J$ Cardiol 2008;129(1):93-9.

50. Evangelista LS, Berg J, Dracup K. Relationship between psychosocial variables and compliance in patients with heart failure. Heart Lung 2001;30(4):294-301.

51. Drozdzowska B, Pluskiewicz W, Skiba M. Knowledge about osteoporosis in a cohort of Polish females: the influence of age, level of education and personal experiences. Osteoporos Int 2004;15(8):645-8.

52. Lee JR, Paultre F, Mosca L. The association between educational level and risk of cardiovascular disease fatality among women with cardiovascular disease. Womens Health Issues 2005;15(2):80-8.

53. Pincus T, Keysor J, Sokka T, Krishnan E, Callahan LF. Patient questionnaires and formal education level as prospective predictors of mortality over 10 years in $97 \%$ of 1416 patients with rheumatoid arthritis from 15 United States private practices. J Rheumatol 2004;31(2):229-34.

54. de Jong Z, Munneke M, Jansen LM, Ronday K, van Schaardenburg DJ, Brand R, et al. Differences between participants and nonparticipants in an exercise trial for adults with rheumatoid arthritis. Arthritis Rheum 2004;51(4):593-600.

55. Thoolen B, de Ridder D, Bensing J, Gorter K, Rutten G. Who participates in diabetes self-management interventions?: Issues of recruitment and retainment. Diabetes Educ 2007;33(3):465-74.

56. Bennett SJ, Sauve MJ. Cognitive deficits in patients with heart failure: a review of the literature. J Cardiovasc Nurs 2003;18(3):219-42. 



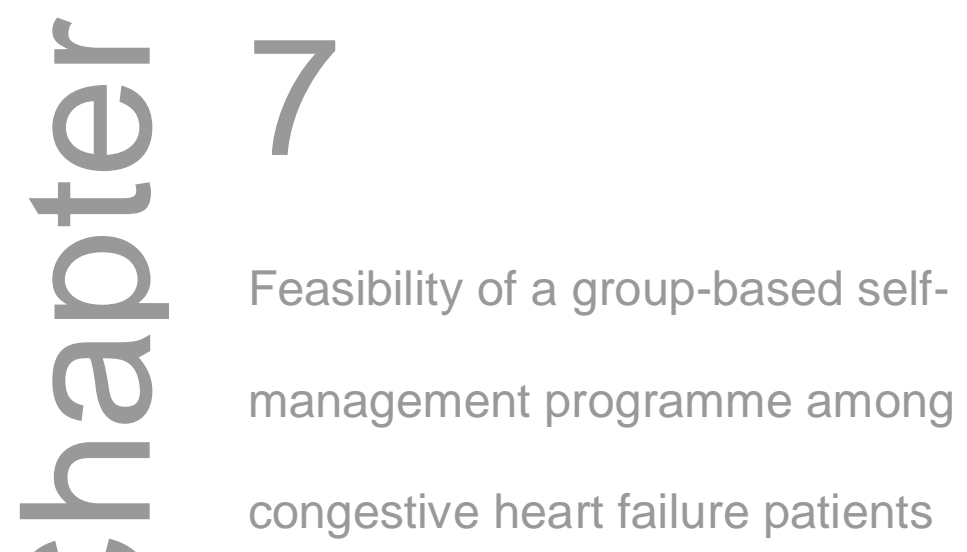

ESTF Smeulders, JCM van Haastregt, JJJ Janssen-Boyne, HEJH Stoffers, JThM van Eijk, GIJM Kempen

Heart \& Lung, in press 


\section{Abstract}

Objective This study assessed the feasibility of the Chronic Disease SelfManagement Programme (CDSMP) among patients with congestive heart failure (CHF). The programme emphasizes patients' central role and responsibility in managing their illness.

Methods Patients were randomly assigned to the programme, which was led by a cardiac nurse specialist and a CHF patient. Data on performance according to protocol, adherence, and opinion about the programme were collected among 186 patients and 18 leaders.

Results Eighty percent of the group sessions were carried out largely according to protocol. Three-fourths of the patients attended at least four of the six sessions. Female sex and lower New York Heart Association (NYHA) classification predicted good attendance.

Conclusion Directly after the programme and at twelve-month of follow-up after the start of the programme, approximately three-fourths of the patients stated that they had benefited from the programme. Recommendations mainly concerned spending more time on several programme topics and specifying patient-selection criteria in more detail. The programme was considered feasible. 


\section{Introduction}

Western societies are experiencing an epidemic of congestive heart failure (CHF) due to better treatment options for patients with acute cardiovascular disease (CVD) and general progressive aging of the population. ${ }^{1-4}$ Clinical trials have demonstrated poor prognosis in patients with $\mathrm{CHF}$, which reflects an optimistic estimate compared with the prognosis in community-based populations. ${ }^{4,5}$ Recently, community-based 1-, 2-, 5-, and 7-year survival rates of $74 \%, 65 \%, 45 \%$ and $32 \%$, respectively, were found for $\mathrm{CHF}$ patients in The Netherlands compared with $97 \%, 94 \%, 80 \%$ and $70 \%$ in a matched reference group of people without CHF. ${ }^{4}$ In addition, CHF has a substantial impact on daily functioning, health-related quality of life, and healthcare utilization, apart from the consequences of aging. ${ }^{6}$

Because CHF touches nearly every important aspect of daily life, patients are engaged to improve their self-management abilities and to take responsibility for day-to-day self-management. According to one of the first definitions, the term 'self-management', which is often used interchangeably with the term 'self-care', indicates patients' active participation in (chronic) disease treatment. ${ }^{7-9}$ In general, CHF self-management comprises four areas: (1) treatment (e.g. adherence to prescribed medications); (2) self-monitoring (e.g. daily weighing); (3) lifestyle modifications (e.g. adhering to diet restriction and exercise); and (4) symptom recognition (e.g. dyspnea, edema). ${ }^{9-12}$ Where patients' exercise intolerance leads to activity restriction and progressive functional deterioration, physical activity may interrupt this downward spiral and improve functional capacity and quality of life. ${ }^{13}$ Diet and lifestyle changes may prevent complications and hospitalization, thus slowing the progression of disease. ${ }^{9,10}$ Furthermore, fatigue management, breathing techniques, and relaxation can play an important role in daily CHF self-management. ${ }^{14}$

Therefore, self-management seems necessarily complementary to professional healthcare for CHF patients. Unfortunately, many CHF patients fail to adhere to the complex treatment regimen. ${ }^{15-17}$ Many intervention programmes have been developed and evaluated to improve selfmanagement behaviour among CHF patients. Some of these - such as telephone- or home-based interventions, ${ }^{18-20}$ programmes in clinical (outpatient) settings, ${ }^{21-23}$ and group-based programmes ${ }^{24-26}$ - have demonstrated positive outcomes on health-related quality of life. However, most intervention programmes solely emphasize the medical aspects of $\mathrm{CHF}$ self-management (e.g. symptom recognition), whereas patients face equal difficulties in dealing with the social and emotional consequences of $\mathrm{CHF}^{27,28}$ 
The Chronic Disease Self-Management Programme (CDSMP) focuses on the medical (i.e. physical), social, and emotional consequences of being chronically ill and emphasizes the patients' central role and responsibility in managing their illness on all three levels. ${ }^{829-31}$ The CDSMP is based on Bandura's ${ }^{32}$ self-efficacy theory and teaches patients three self-management skills: (1) how to manage the medical consequences of the disease (medical management, e.g. medication adherence and lifestyle changes, such as exercising regularly); (2) how to remain socially active in daily life (role management); and (3) how to manage the emotional consequences of being chronically ill (emotional management). ${ }^{31}$ Currently the CDSMP is a well known and widely disseminated group-based programme aimed at helping people with various chronic conditions to achieve a positive and active selfmanagement lifestyle by enhancing their self-efficacy expectancies. ${ }^{33-39}$ Previous studies have found favourable effects of the CDSMP on outcomes, such as self-efficacy expectancies, health behaviour, and hospitalization, in heterogeneous groups of patients with chronic diseases, including CVD patients (e.g. coronary artery disease and $\mathrm{CHF}$ ) and patients with chronic lung disease (e.g. asthma and emphysema). ${ }^{8,29-31}$ The effectiveness and feasibility of the programme, however, have not yet been assessed among a group comprised solely of CHF patients.

We expect that $\mathrm{CHF}$ patients can benefit from this self-management programme because increasing self-management skills in this group of patients may positively influence their self-care, their health-related quality of life (i.e. physical, social, and emotional functioning), and their healthcare utilization. Furthermore, lower levels of self-efficacy expectancies seem to predict functional decline in CHF patients, which could be altered by attending the programme. ${ }^{40}$ Finally, by emphasizing social and emotional aspects of self-management as well, the programme is based on a more holistic definition of self-management, which corresponds to a greater level of the day-to-day management of CHF than most self-management programmes that have been previously tested among CHF patients. In fact, psychosocial factors may even be the key in effective CHF management. ${ }^{27,28,41}$ Although the CDSMP was originally developed for use in heterogeneous patient groups, the programme also seems applicable to a more homogeneous setting of CVD patients. ${ }^{42}$ Therefore, we assessed the effects of the CDSMP on psychosocial attributes (e.g. self-efficacy expectancies), health behaviour (e.g. self-care), quality of life, and healthcare utilization solely among CHF patients in a randomized controlled trial $(n=317) .{ }^{43} \mathrm{~A}$ detailed process evaluation was performed alongside this trial to assess the feasibility of the CDSMP among patients and leaders and to assess the factors predicting 
good attendance in this population. CHF patients often experience decreased exercise capacity, which may negatively influence attendance. Based on the results of this process evaluation, we make recommendations for increasing programme feasibility (if necessary) and improving patients' attendance to facilitate implementation of the programme in regular outpatient care (i.e. regular checkups with the cardiologist and/or the CHF nurse specialist).

\section{Methods}

\section{Participants}

The process evaluation was performed among $186 \mathrm{CHF}$ patients (who were assigned to one of the 21 CDSMP classes) and 18 leaders (nine 'professional leaders' and nine 'peer leaders') who conducted the programme during the intervention period after they had been trained for four days on the CDSMP protocol. ${ }^{43}$ The professional leaders were cardiac nurse specialists. They conducted the CDSMP classes in combination with their usual work in the hospital. The peer leaders were CHF patients who had New York Heart Association (NYHA) Class II and III symptoms. The patients in the study were selected from the heart failure and/or cardiology outpatient clinics of six participating hospitals in the southern part of The Netherlands from October 2004 to January 2006. The hospitals were situated in a range of $86 \mathrm{~km}$ from the study site and were comparable with regard to clinical and outpatient care facilities (i.e. regular checkups with the cardiologist and/or the CHF nurse specialist) for CHF patients with NYHA Class II and III symptoms. Patients were eligible for the study if they had CHF for at least six months based on systolic or diastolic dysfunction (plus an additional hospital admission for decompensatio cordis after $\mathrm{CHF}$ diagnosis based on diastolic dysfunction) and experienced a slight to marked limitation of physical activity (NYHA Class II and III symptoms). There was no exclusion based on time interval from $\mathrm{CHF}$ diagnosis. A more detailed description of the selection of the patients and training of the leaders has been published elsewhere. ${ }^{43}$

\section{Intervention}

The CDSMP is a structured group-based programme addressing general management problems for patients with chronic conditions. The programme consists of six weekly sessions (of two and a half hours per session) and 
teaches patients to become active self-managers in medical, role, and emotional management. ${ }^{31}$ Regarding medical management, topics such as medication usage, making informed treatment decisions, and making a personal exercise plan are addressed. Topics to improve remaining socially active are, for example, action-planning and communication skills. Finally, topics such as dealing with anger, fear, and frustration and depression management are addressed to focus on emotional management issues. Table 1 lists the CDSMP activities for each session. ${ }^{44}$

Table 1: Contents of the CDSMP and mean time spent per session

\begin{tabular}{|c|c|c|}
\hline Activity 1 & Introduction - Identifying common problems & Session 1 (scheduled 140 \\
\hline Activity 2 & Workshop overview and responsibilities & minutes) \\
\hline Activity 3 & Differences between acute and chronic conditions & Observed mean $=145$ minutes \\
\hline Activity 4 & Introduction to cognitive symptom management & (range $120-180$ ) \\
\hline Activity 5 & Introduction to action plans & \\
\hline Activity 6 & Closing & \\
\hline Activity 1 & Feedback/problem-solving session & Session 2 (scheduled 135 \\
\hline Activity 2 & Dealing with emotions (anger, fear, frustration) & minutes) \\
\hline Activity 3 & Introduction to exercise & Observed mean $=142$ minutes \\
\hline Activity 4 & Making an action plan & (range $120-160$ ) \\
\hline Activity 5 & Closing & \\
\hline Activity 1 & Feedback/problem-solving session & Session 3 (scheduled 130 \\
\hline Activity 2 & Better breathing & minutes) \\
\hline Activity 3 & Muscle relaxation & Observed mean $=134$ minutes \\
\hline Activity 4 & Fatigue management & (range $100-150)$ \\
\hline Activity 5 & Endurance exercise & \\
\hline Activity 6 & Making an action plan & \\
\hline Activity 7 & Closing & \\
\hline Activity 1 & Feedback/problem-solving/making an action plan & Session 4 (scheduled 145 \\
\hline Activity 2 & Healthy eating & minutes) \\
\hline Activity 3 & Distraction & Observed mean $=149$ minutes \\
\hline Activity 4 & Advance directives for healthcare & (range $130-180$ ) \\
\hline Activity 5 & Communication skills & \\
\hline Activity 6 & Problem-solving & \\
\hline Activity 7 & Closing & \\
\hline Activity 1 & Feedback/problem-solving/making an action plan & Session 5 (scheduled 140 \\
\hline Activity 2 & Medication usage & minutes) \\
\hline Activity 3 & Making informed treatment decisions & Observed mean $=140$ minutes \\
\hline Activity 4 & Depression management & (range $120-180$ ) \\
\hline Activity 5 & Self-talk & \\
\hline Activity 6 & Guided imagery & \\
\hline Activity 7 & Closing & \\
\hline Activity 1 & Feedback/problem-solving & Session 6 (scheduled 125 \\
\hline Activity 2 & Informing the healthcare team & minutes) \\
\hline Activity 3 & Working with your healthcare professional & Observed mean $=132$ minutes \\
\hline Activity 4 & Looking back and planning for the future & (range $115-165)$ \\
\hline Activity 5 & Closing & \\
\hline
\end{tabular}


Self-efficacy is considered the key mechanism of the CDSMP because selfefficacy and self-efficacy expectancies are associated with changes in health behaviour and health status. ${ }^{8,32}$ To enhance self-efficacy expectancies, the programme incorporates four strategies, namely skills mastery, reinterpretation of symptoms, modelling, and social persuasion. ${ }^{8,31}$ Skills mastery includes goal-setting, making weekly action plans, and feedback, which takes up approximately $25 \%$ to $35 \%$ of each session. The programme aims at motivating patients to make behaviour changes by setting personal goals and breaking them down into smaller steps (action plans). At each session, the patients in the group are invited to make their own personal, yet reasonable, action plan, which must be achieved before the next session, e.g. walking three blocks after dinner every other day. To check reasonability of the action plan and maximally improve the chance for success, patients are questioned plenary about their confidence level to entirely complete their action plan. If the confidence level is too low ( $<7$ on a scale of 0 to 10), problem-solving of barriers takes place, and the action plan is adjusted.

During the next session, the action plans for the past week are discussed, and problem-solving takes place in a group brainstorm exercise if participants failed to accomplish their action plan. During each session, patients are invited to make a new weekly action plan. Goal-setting and action-planning is encouraged during the sessions; however, the participants' decision to make and complete an action plan is entirely voluntary, which emphasizes the patients' responsibility in managing their chronic condition in daily life. ${ }^{44}$ Patients are supported in action-planning through supportive telephone calls from co-participants. Furthermore, patients are encouraged to set an exercise goal and to create a personal exercise programme during the programme.

Reinterpreting symptoms comprises cognitive symptom management techniques and deals with modifying patients' beliefs about their symptoms to help to relieve symptom problems. The modelling and social-persuasion aspects of the programme support and motivate patients to make these changes in their behaviours and beliefs, e.g. those patients who are sceptical about making action plans. ${ }^{44}$ In addition, Lorig and colleagues developed the reference book Living a healthy life with chronic conditions. ${ }^{14}$ This book was translated into Dutch together with the programme protocol. ${ }^{45}$ The patients received this book during the first programme session. During each session, patients were invited to read a part of the book covering the contents of that particular session.

In our study, all CDSMP classes were led by a team comprising a cardiac nurse specialist ('professional leader') and a CHF patient ('peer leader'), who acted as a role model for the other CHF patients. Normally, CDSMP classes 
are led by two trained volunteer lay leaders (who may be chronically ill). However, in light of future implementation of the programme in Dutch healthcare, we expect that including a professional leader will facilitate this implementation. Furthermore, because $\mathrm{CHF}$ is a serious and life-threatening disease, CHF peer leaders may only be able to conduct the programme for a limited period, which could form a serious problem regarding continuity of the programme. The format and contents of the intervention protocol, as well as the design of the randomized controlled trial, have been described in more detail elsewhere. ${ }^{43}$

\section{Measurements}

To determine the programme's feasibility, three main outcome measures were assessed among patients and/or leaders: (1) the extent to which the programme was performed according to protocol; (2) patients' adherence to the programme; and (3) leaders' and participants' opinions about the programme. Data from the patients who attended at least half of the programme were collected directly after the programme and again one year later by means of self-administered questionnaires. Data from the leaders were collected during and directly after the programme by means of recording forms and self-administered questionnaires (Table 2).

Table 2: Feasibility measures at baseline and during follow-up

\begin{tabular}{|c|c|c|c|}
\hline Process evaluation outcome variables & BDP & FU1 & FU2 \\
\hline \multicolumn{4}{|l|}{ Performance according to protocol } \\
\hline Duration of each session & $\mathrm{RF}^{\prime}$ & - & - \\
\hline Deviations from protocol & $\mathrm{RF}^{\prime}$ & - & - \\
\hline \multicolumn{4}{|l|}{ Patients' attendance } \\
\hline Reasons for refusal before starting the programme & $\mathrm{Tl}^{\mathrm{p}}$ & - & - \\
\hline Number of sessions attended by each patient & $\mathrm{RF}^{\prime}$ & - & - \\
\hline Reasons for stopping during the programme & $\mathrm{TI}^{\mathrm{p}}$ & - & - \\
\hline \multicolumn{4}{|l|}{ Patients' adherence } \\
\hline Overall adherence & $\mathrm{RF}^{\prime}$ & - & - \\
\hline Adherence to homework assignments & - & $Q^{p} / Q^{\prime}$ & - \\
\hline \multicolumn{4}{|l|}{ Opinion about the programme } \\
\hline Extent of benefits from the programme & - & $Q^{p}$ & $Q^{p}$ \\
\hline Strong and weak points of the programme (sessions) & $\mathrm{RF}^{\prime}$ & $Q^{p} / Q^{\prime}$ & - \\
\hline General opinion about the quality of the leaders & - & $Q^{p} / Q^{\prime}$ & - \\
\hline General opinion about the programme & $\mathrm{RF}^{\prime}$ & $\mathrm{Q}^{\mathrm{p}} / \mathrm{Q}^{\mathrm{l}}$ & - \\
\hline Recommending the programme to other patients & - & $Q^{p}$ & - \\
\hline Perceived benefits (programme contributions) & - & $Q^{p} / Q^{\prime}$ & $Q^{\mathfrak{p}}$ \\
\hline Recommendations for improvement & - & $Q^{p} / Q^{\prime}$ & - \\
\hline
\end{tabular}


In addition, the leaders discussed programme experiences in bi-monthly structured meetings and in a structured group interview at the end of the intervention period using semistructured evaluation forms.

\section{Performance according to protocol}

Regarding performance according to protocol, the leaders recorded for each session whether they deviated from protocol (e.g. exceeded specified time limits per activity or skipped activities). In addition, they recorded preparation time and time spent on the session itself.

\section{Patients' adherence to the programme}

Patients' adherence to the programme was assessed by measuring patients' attendance, overall patients' adherence during the sessions, and patients' adherence to homework assignments. During each session, the leaders reported on patients' attendance. Absent patients were called on the phone by the professional leaders to ask the reason for absence. Reasons for withdrawal before the start of the intervention or for stopping during the intervention period were also recorded. In addition, for each session the leaders reported on overall adherence to the programme (i.e. patients' efforts during the programme sessions) at the group level (5-point scale: 'very good' to 'very poor'). Directly after completing the programme, both leaders and patients reported on the adherence to homework assignments. The patients reported on the number of action plans which they created and accomplished successfully during the programme and reported whether they made a personal exercise programme. The leaders reported on the number of patients who developed weekly action plans and accomplished them successfully (both 4-point scales: 'most participants in the group' to 'nobody in the group').

\section{Patients' and leaders' opinions about the programme}

Directly after the programme, patients were asked to what extent they had benefited from the programme (5-point scale: 'did not benefit' to 'benefited very much') and which parts of the programme they did or did not appreciate. In addition, patients were asked (1) to give report marks for the quality of the 
professional and peer leaders as well as the programme itself (range 1 [low] to 10 [high]); (2) whether they would recommend the programme to other $\mathrm{CHF}$ patients or to patients with other chronic diseases (e.g. diabetes mellitus); and (3) whether the programme contributed to improvement of specific health behaviours and coping strategies ('perceived benefits'), compared with before they attended the programme (2-point scale: 'yes' or 'no'). Finally, the patients were asked for suggestions to improve the programme. After one year, the patients received a short questionnaire to re-evaluate the CDSMP (e.g. 'perceived benefits') in the previous twelve months.

The leaders gave report marks for each session and for the programme as a whole, and they reported on the strengths and weaknesses of the sessions. In addition, the leaders gave a report mark for their own leadership skills, gave their opinion about patients' perceived benefits of the programme (3-point scale: 'most participants in the group improved' to 'nobody in the group improved'), and made recommendations for improvement to the programme. Finally, the leaders evaluated the feasibility of the protocol (e.g. regarding co-leadership and session duration and frequency) and made recommendations to improve the programme in a structured group interview at the end of the intervention period.

\section{Data analysis}

Data were analysed by means of descriptive statistics. Regarding the prediction of attendance patients who attended at least four sessions (i.e. high attenders) were compared with patients who attended less than four sessions (i.e. low attenders). Three types of variables, which had been assessed at baseline, were used to assess attendance to the programme: (1) sociodemographic variables (sex, age, living arrangements, and educational level); (2) health-related variables (health-related quality of life, self-care behaviour, cognitive status, and NYHA classification); and (3) psychosocial variables (symptoms of anxiety and feelings of depression, self-efficacy expectancies, and perceived control). These measures have been described in more detail elsewhere. ${ }^{43}$ Univariate analyses were conducted to compare both groups on socio-demographic, health-related, and psychosocial variables using Student $t$ test for independent samples, chi-square test, and Mann-Whitney $\mathrm{U}$ test. Characteristics of the leaders, i.e. sex, age, and educational level, were obtained. In addition, NYHA classification and time interval from CHF diagnosis were obtained from the CHF peer leaders. The analyses were performed using SPSS for Windows, version 12.0.1. 


\section{Results}

\section{Participant characteristics}

Mean patient age ( $n=186)$ was 66.6 years (SD 11.0; range 29 to 87). The majority of patients were men $(76 \%[n=141])$, did not live alone $(67 \%)$, were unemployed (91\%), and had a secondary (vocational) education (64\%). Approximately two-thirds of patients (65\%) experienced NYHA Class II symptoms (slight physical limitation). The majority of the professional leaders were female $(78 \%[n=7])$, and all peer leaders were male; mean ages were 45.6 (SD 5.2) and 58.1 (SD 10.5) years, respectively. Professional leaders had a tertiary (vocational) education, and the majority of the peer leaders had a secondary/tertiary (vocational) education (77.7\%). Eight of nine peer leaders experienced NYHA Class II symptoms, and the mean time interval from CHF diagnosis was 12.4 months (SD 6.3; range 2.5 to 22.4). The results on leader characteristics are not tabulated.

\section{Performance according to protocol}

The mean number of participants allocated per class was 8.9 (range 7 to 13). The actual mean number of participants (excluding patients who attended no sessions) per class was 7.8 (range 6 to 12). The leaders completed 241 of the 252 ( 21 classes $\times 6$ sessions $\times 2$ leaders; $96 \%$ ) recording forms and 40 of the 42 ( 21 classes $\times 2$ leaders; $95 \%$ ) self-administered questionnaires. Two peer leaders were unable to attend the structured group interview at the end of the intervention period. Regarding performance according to protocol, 117 of the 126 sessions ( 21 classes $\times 6$ sessions; 93\%) were led by a professional leader and a peer leader. The other sessions were conducted by a professional leader only because the peer leader was absent due to illness or other reasons, which happened at least one time (range 1 to 3 ) in five CDSMP classes. An absent peer leader was not replaced unless this occurred during the first session of the programme. This exception was made because of the modelling role of the peer leader during the first session in particular. In addition, leaders stated that 101 of the 126 sessions (80\%) were carried out according to protocol. Only small protocol deviations were reported, with the exception being session four during which, in 12 of the 21 CDSMP classes, the activities 'advance directives for healthcare' (e.g. in an end-of-life situation) and 'communication skills' overran the allotted time in the protocol. These activities were considered difficult topics to comprehend or to focus on by the participants. This resulted in not performing the next activity 
'problem-solving' according to protocol or skipping it because of lack of time or participant motivation. In the other nine classes, no substantial deviations were mentioned in session four. Five of six sessions took more time on average than allotted per the protocol (Table 1). However, our CDSMP classes were smaller, therefore, more time could be spend per participant compared with the amount of time per class reported by Lorig and colleagues. $^{29-31}$ The mean time per session was 140 minutes, which corresponded well with the time allotted per protocol (calculated mean 136). Furthermore, the range of duration of the sessions varied substantially (100 to 180 minutes), whereas the duration of sessions per protocol varied from 125 to 145 minutes (Table 1). Mean preparation time was 46 minutes per session per leader (range 0 to 180). In general, peer leaders needed slightly more time to prepare for a session than professional leaders (52 versus 40 minutes, respectively). The amount of preparation time depended on the leader's protocol routine and decreased during the intervention period of the trial.

\section{Patients' adherence to the programme}

\section{Patients' attendance}

In total $79 \%$ of the patients $(n=146)$ attended at least half of the programme, and $47 \%(n=88)$ attended all six sessions of the programme. Of the 146 patients who attended at least half of the programme, 138 patients (95\%) completed the first evaluation form to assess adherence to home work assignments and gave their opinion about the programme. After one year, $86 \%(n=126)$ of the patients responded to the second evaluation form. Table 3 lists the main findings regarding patients' attendance.

Overall patients' adherence to the programme and home work assignments Patients' adherence to the programme was '(very) good' in $90 \%$ of the sessions (Table 3). Regarding home work assignments, the leaders reported that 'most participants in the group' made weekly action plans and successfully accomplished these action plans on a weekly basis as well (not tabulated). Of the patients who made a personal exercise programme during the programme, $46 \%(n=58)$ was still exercising according to their personal exercise programme at one year after the programme (Table 3 ). 
Table 3: Patients' adherence to the CDSMP

\begin{tabular}{|c|c|c|c|}
\hline $\begin{array}{l}\text { Patients' adherence } \\
\text { Attendance }(n=186)(\%)\end{array}$ & \multicolumn{3}{|c|}{ Main findings regarding adherence at FU1 } \\
\hline Attendance $(n=186)(\%)$ & $\begin{array}{l}0 \text { sessions } \\
\geq 1 \text { sessior } \\
\geq 3 \text { sessior } \\
\geq 4 \text { sessior } \\
\text { All } 6 \text { sessic } \\
\text { Mean total } \\
\text { Mean parti }\end{array}$ & $\begin{array}{l}\text { attendance) } \\
\text { ttendance } \\
\text { tendance* }\end{array}$ & $\begin{array}{l}12(n=23) \\
88(n=163) \\
79(n=146) \\
75(n=140) \\
47(n=88) \\
4.4 \text { sessions } \\
5.0 \text { sessions }\end{array}$ \\
\hline $\begin{array}{l}\text { Reasons for refusal before starting } \\
\text { the programme }(n=23)\end{array}$ & $\begin{array}{l}\text { Health prob } \\
\text { No time/op } \\
\text { Motivatione } \\
\text { Emotional } \\
\text { Death } \\
\text { Other reas }\end{array}$ & ms & $\begin{array}{l}n=12 \\
n=6 \\
n=1 \\
n=1 \\
n=1 \\
n=2\end{array}$ \\
\hline $\begin{array}{l}\text { Reasons for stopping during the } \\
\text { programme }(n=17)\end{array}$ & $\begin{array}{l}\text { Health prok } \\
\text { Motivation } \\
\text { No time/op } \\
\text { Emotional } \\
\text { Cognitive p } \\
\text { Other reas }\end{array}$ & ms & $\begin{array}{l}n=8 \\
n=5 \\
n=1 \\
n=1 \\
n=1 \\
n=1\end{array}$ \\
\hline $\begin{array}{l}\text { Overall (\%) adherence ( } n=241 \\
\text { leader session observations) }\end{array}$ & $\begin{array}{l}\text { Very good } \\
\text { Good }\end{array}$ & $\begin{array}{l}38(n=91) \\
52(n=125)\end{array}$ & \\
\hline Patients' action plans $(n=135)(\%)$ & $\begin{array}{l}\text { Mean } \\
\geq 4 \text { plans }\end{array}$ & $\begin{array}{l}4.4 \\
77(n=104)\end{array}$ & \\
\hline $\begin{array}{l}\text { Patients' action plans accomplished } \\
(n=132)(\%)\end{array}$ & $\begin{array}{l}\text { Mean } \\
\geq 4 \text { plans }\end{array}$ & $\begin{array}{l}3.8 \\
64(n=85)\end{array}$ & \\
\hline $\begin{array}{l}\text { Number }(\%) \text { of patients who made an } \\
\text { exercise programme }(n=137)\end{array}$ & & $81(n=111)$ & \\
\hline $\begin{array}{l}\text { Patients }(\%) \text { exercising according to } \\
\text { plan }(n=135)\end{array}$ & FU1 & $78(n=105)$ & \\
\hline $\begin{array}{l}\text { Patients (\%) exercising according to } \\
\text { plan }(n=125)\end{array}$ & FU2 & $46(n=58)$ & \\
\hline $\begin{array}{l}\text { Content }(\%) \text { of exercise programme } \\
(n=105)\end{array}$ & $\begin{array}{l}\text { Walking: } 2 \\
\text { Walking an } \\
\text { W/b/s in co }\end{array}$ & $\begin{array}{l}\text { cling and/or } \\
\text { on with physic }\end{array}$ & 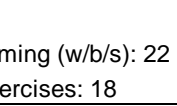 \\
\hline
\end{tabular}

FU1 = follow-up directly after the programme $(n=138) ;$ FU2 = follow-up at 12 months after the programme $(n=126) ;{ }^{*} N=163$ (attended $\geq 1$ session).

\section{Patients' and leaders' opinions about the programme}

\section{Patients' opinions about the programme}

Tables 4 to 6 list the main findings with regard to opinions about and perceived benefits of the programme. Overall, most patients (73\%) reported directly after the programme having 'benefited (relatively) much' from the sessions. They gave a mean report mark of 8.1 for the programme. Thirtyeight patients first mentioned action-planning as a strong point of the 
programme. In contrast, 30 patients stated that they did not appreciate the cognitive symptom management techniques, particularly guided imagery, which was a guided imagination for relaxation; that they were already familiar with most of the content; or that they should have attended the programme earlier in their illness process. Patients were positive about the quality of the leaders, and the professional leaders received a somewhat higher overall score (8.3) than the peer leaders (7.9) (Table 4).

Table 4: Patients' opinions and recommendations

\begin{tabular}{|c|c|c|}
\hline Programme opinions & Main findings regarding patients' & at FU \\
\hline $\begin{array}{l}\text { Benefited from CDSMP } \\
(n=138)(\%)\end{array}$ & $\begin{array}{l}\text { Much } \\
\text { Relatively much }\end{array}$ & $\begin{array}{l}37 \%, n=51 \\
36 \%, n=50\end{array}$ \\
\hline $\begin{array}{l}\text { Benefited from CDSMP } \\
\text { at FU2 }(n=126)(\%)\end{array}$ & $\begin{array}{l}\text { Much } \\
\text { Relatively much }\end{array}$ & $\begin{array}{l}33 \%, n=42 \\
41 \%, n=54\end{array}$ \\
\hline Strong points $(n=127)(\%)$ & $\begin{array}{l}\text { Action-planning } \\
\text { Cognitive symptom management } \\
\text { Exercise activities } \\
\text { All activities }\end{array}$ & $\begin{array}{l}30 \%, n=38 \\
14 \%, n=18 \\
13 \%, n=16 \\
10 \%, n=13\end{array}$ \\
\hline Weak points $(n=122)(\%)$ & $\begin{array}{l}\text { Cognitive symptom management } \\
\text { Already familiar with content/should } \\
\text { have attended programme earlier } \\
\text { Advance directives } \\
\text { No weak points }\end{array}$ & $\begin{array}{l}15 \%, n=18 \\
10 \%, n=12 \\
10 \%, n=12 \\
30 \%, n=36\end{array}$ \\
\hline Programme content $(n=137)$ & Mean report mark & 8.1 (range 5-10) \\
\hline $\begin{array}{l}\text { Recommend programme } \\
(n=137)(\%)\end{array}$ & $\begin{array}{l}\text { To other CHF patients } \\
\text { To patients with other disease }\end{array}$ & $\begin{array}{l}96 \%, n=132 \\
94 \%, n=128\end{array}$ \\
\hline Quality leaders $(n=137)$ & $\begin{array}{l}\text { Peer: mean report mark } \\
\text { Professional: mean report mark }\end{array}$ & $\begin{array}{l}7.9 \text { (range } 4-10) \\
8.3 \text { (range 5-10) }\end{array}$ \\
\hline $\begin{array}{l}\text { Recommendations for the } \\
\text { programme }(n=107)\end{array}$ & $\begin{array}{l}\text { Format } \\
\text { Session/patient number adjustments } \\
\text { with } 8-10 \text { patients/session }(n=5) \\
\text { Booster sessions: } 6 \text { monthly meeting } \\
\text { discuss progress and programme be } \\
\text { Leaders: do not conduct sessions wi } \\
\text { Content } \\
\text { (More) time on specific topics: advan } \\
\text { medication usage ( } n=14 \text { ) } \\
\text { Inviting experts: dietician, cardiologis } \\
\text { Book improvements: run book analo } \\
\text { insert tabs between chapters ( } n=3 \text { ) } \\
\text { Target group } \\
\text { Patient characteristics: account for la } \\
\text { Co-participants: partner to improve u } \\
\text { disease, family member }(n=6) \\
\text { Time interval from CHF diagnosis: af } \\
\text { hospital, } 6 \text { months after diagnosis ( } n \\
\text { No recommendations }(n=48)\end{array}$ & $\begin{array}{l}\text { ions } 1.5 \text {-hour sessions } \\
\text { aders to } \\
=4) \\
\text { eer leader }(n=4) \\
\text { ives, healthy eating, } \\
\text { al therapist }(n=4) \\
\text { th programme; } \\
\text { differences ( } n=7) \\
\text { ding complexity } \\
\text { dmission to }\end{array}$ \\
\hline
\end{tabular}

FU1 = follow-up directly after the programme $(n=138) ;$ FU2 = follow-up at 12 months after the programme $(n=$ $126) ;{ }^{*} N=136$. 
A majority of the patients confirmed that the programme had improved specific health behaviours and coping strategies. More than $80 \%$ of the patients perceived themselves as being able to improve their physical condition, to use action plans, to deal with their condition(s) more effectively, and to exercise more after they had attended the programme (Table 5). Finally, the patients were asked to suggest improvements to the programme, and most addressed the format of the CDSMP, the protocol content, and the group of patients to which the programme was targeted. Most comments were given on spending more time on specific topics $(n=14)$; taking patients' different characteristics into account because patients experienced rather large differences between themselves and other patients in the class $(n=7)$; and inviting partners or family members to attend the programme $(n=6)$ (Table 4).

After one year, the majority of the patients confirmed their opinion that the programme was still contributing positively to their specific health behaviours and coping strategies. However, the number of patients who thought that the programme was still contributing to their using action plans and exercising more was considerably decreased (58\% versus $82 \%$ and $69 \%$ versus $81 \%$, respectively; Table 5).

In contrast, patients reported that the programme was still (and even slightly more) contributing to sensible medicine use, following diet instructions, and using cognitive symptom management techniques to lessen physical complaints one year later (> 5\% improved percentages). Overall, $75 \%$ of the patients still 'benefited (relatively) much' from the programme at one year.

Other findings were that in the past year, on average, the patients occasionally used the book, Living a healthy life with chronic conditions, ${ }^{14}$ as a reference source, and $12 \%$ of the patients reported having made and kept contact with one or more of the participants after completing the programme. Finally, at least half of the patients $(n=69)$ reported they would prefer a booster session at six months (48\%) or at twelve months (49\%) after attending the programme (not tabulated). 
Table 5: Contributions of the CDSMP according to patients and leaders

\begin{tabular}{|c|c|c|c|c|c|c|}
\hline \multirow[b]{3}{*}{$\begin{array}{l}\text { Improved health } \\
\text { behaviours/coping } \\
\text { strategies }\end{array}$} & \multicolumn{4}{|c|}{ Data collected from patients } & \multirow{2}{*}{\multicolumn{2}{|c|}{$\begin{array}{c}\begin{array}{c}\text { Data collected } \\
\text { from leaders }\end{array} \\
\text { Agreed (FU1) }\end{array}$}} \\
\hline & \multicolumn{2}{|c|}{ Agreed (FU1) } & \multicolumn{2}{|c|}{ Agreed (FU2) } & & \\
\hline & $\%(n)$ & $\begin{array}{l}\text { Analysed } \\
(n)\end{array}$ & $\%(n)$ & $\begin{array}{l}\text { Analysed } \\
\text { (n) }\end{array}$ & $\%(n)$ & $\begin{array}{l}\text { Analysed } \\
(n)\end{array}$ \\
\hline $\begin{array}{l}\text { Being able to improve my } \\
\text { physical condition in a sensible } \\
\text { way }\end{array}$ & $86(115)$ & 134 & $77(96)$ & 125 & $73(40)$ & 40 \\
\hline $\begin{array}{l}\text { Using action plans to reach my } \\
\text { long-term goals }\end{array}$ & $82(111)$ & 135 & $58(73)$ & 125 & $60(40)$ & 40 \\
\hline $\begin{array}{l}\text { Dealing with my condition(s) } \\
\text { more effectively in daily life }\end{array}$ & $81(110)$ & 135 & $83(104)$ & 125 & $75(40)$ & 40 \\
\hline Exercising more & $81(109)$ & 134 & $69(86)$ & 125 & $73(40)$ & 40 \\
\hline $\begin{array}{l}\text { Taking my medicines in a } \\
\text { sensible way }\end{array}$ & $74(99)$ & 134 & $81(101)$ & 125 & $70(40)$ & 40 \\
\hline $\begin{array}{l}\text { Discussing problems or unclear } \\
\text { issues with my doctor and } \\
\text { healthcare providers more } \\
\text { effectively }\end{array}$ & $73(99)$ & 135 & $77(96)$ & 124 & $55(40)$ & 40 \\
\hline $\begin{array}{l}\text { Coping with unpleasant } \\
\text { emotions or thoughts more } \\
\text { effectively }\end{array}$ & $73(96)$ & 131 & $75(94)$ & 125 & $65(40)$ & 40 \\
\hline $\begin{array}{l}\text { Feeling more responsible for } \\
\text { managing my condition(s) }\end{array}$ & $73(98)$ & 135 & $72(90)$ & 125 & $78(40)$ & 40 \\
\hline $\begin{array}{l}\text { Being able to follow diet } \\
\text { instructions regarding my } \\
\text { condition(s) more effectively }\end{array}$ & $72(96)$ & 134 & $78(97)$ & 124 & $55(40)$ & 40 \\
\hline $\begin{array}{l}\text { Discussing problems or unclear } \\
\text { issues with my family, friends } \\
\text { and/or acquaintances more } \\
\text { effectively }\end{array}$ & $65(87)$ & 133 & $58(72)$ & 125 & $58(40)$ & 40 \\
\hline $\begin{array}{l}\text { Lessening physical complaints } \\
\text { by using cognitive symptom } \\
\text { management techniques }\end{array}$ & $62(83)$ & 133 & $67(84)$ & 125 & $43(40)$ & 40 \\
\hline
\end{tabular}

FU1 = follow-up directly after the programme; FU2 = follow-up after 12 months after the programme;

* Percentage of leaders who agreed that "most participants in the group improved this health behaviour/coping strategy as a result of attending the programme".

\section{Leaders' opinions about the programme}

Both professional and peer leaders had favourable opinions about leading the CDSMP together. They each had complementary tasks during the programme sessions: the professional leaders responded to medical issues, whereas peer leaders shared personal experiences and motivated participants to share their own personal experiences. The main strength of the programme sessions was considered the patients' positive and active attitudes during the sessions $(n=91)$. The main weakness of the programme was related to 
group dynamics: managing specific disturbing patients and/or the group in general $(n=54$; Table 6$)$. On average, $(64 \%$ of the classes; range $43 \%$ to $78 \%$; Table 5), leaders reported that according to their perception, most patients in the group had benefited from the programme. However, when comparing these results with the patients' perceived benefits, in general they were more cautious in their opinions than were the patients themselves. Finally, leaders made some recommendations regarding the CDSMP format, the protocol content, and the group of patients to which the programme was targeted. Most recommendations addressed adjustments of specific activities $(n=7)$, taking patients' different characteristics into account $(n=5)$, and taking additional time for individual input from patients $(n=4)$. Based on the results of the structured group interview about the CDSMP format, the majority of the leaders $(n=14)$ did not suggest improving the duration and frequency of the sessions per protocol. Ten leaders suggested having a maximum of ten participants per group, whereas other leaders preferred larger groups of participants to improve patients' interaction during the programme (not tabulated).

Table 6: Leaders' opinions and recommendations

\begin{tabular}{|c|c|c|}
\hline \multirow{2}{*}{$\begin{array}{l}\text { Programme opinions } \\
\text { Strong points } \\
(n=215)(\%)\end{array}$} & \multicolumn{2}{|c|}{ Main findings regarding leaders' opinions at FU1* } \\
\hline & $\begin{array}{l}\text { Positive/active attitude participants } \\
\text { Social support among participants } \\
\text { Leaders' modelling behaviour } \\
\text { Patients' action-planning }\end{array}$ & $\begin{array}{l}42 \%, n=91 \\
18 \%, n=39 \\
11 \%, n=24 \\
6 \%, n=12\end{array}$ \\
\hline $\begin{array}{l}\text { Weak points } \\
(n=191)(\%)\end{array}$ & $\begin{array}{l}\text { Managing disturbing patients/group in general } \\
\text { Teaching problems } \\
\text { No weak points }\end{array}$ & $\begin{array}{l}28 \%, n=54 \\
20 \%, n=38 \\
38 \%, n=73\end{array}$ \\
\hline $\begin{array}{l}\text { Quality leaders } \\
(n=40)(\%)\end{array}$ & Good & $60 \%, n=24$ \\
\hline $\begin{array}{l}\text { Mean report mark on pro } \\
\text { Mean report mark on ses }\end{array}$ & $\begin{array}{l}\text { mme level }(n=40) \\
n \text { level }(n=235)\end{array}$ & $\begin{array}{l}7.7 \text { (range 6-9) } \\
7.7 \text { (range 6-10) }\end{array}$ \\
\hline $\begin{array}{l}\text { Recommendations for } \\
\text { the programme }(n=30)\end{array}$ & $\begin{array}{l}\text { Format } \\
\text { Attendance: financial consequences if absent } \\
\text { before beginning programme }(n=3) \\
\text { Leaders: increase teaching experience with prc } \\
\text { strictly according to protocol }(n=3) \\
\text { Content } \\
\text { Communication and advance directives: simpli } \\
\text { standardized advance directives form }(n=7) \\
\text { Healthy eating: inviting dietician; provide list wi } \\
\text { ( } n=2) \\
\text { More time for patients input: add topics by requ } \\
\text { personal experiences ( } n=4) \\
\text { Target group } \\
\text { Patient characteristics: account for large age d } \\
\text { No recommendations }(n=13)\end{array}$ & $\begin{array}{l}\text { goal-setting } \\
\text { e, teach less } \\
\text { ties, include } \\
\text { exchange } \\
\text { es }(n=5)\end{array}$ \\
\hline
\end{tabular}

\footnotetext{
${ }^{*} \mathrm{FU} 1$ = follow-up directly after the programme.
} 


\section{Determinants of attendance}

In total, 140 of the 186 patients $(75 \%)$ attended at least four of the six sessions; 46 patients attended less than four sessions. The percentage of women was significantly greater among patients who attended at least four sessions compared with patients who attended less than four sessions $(28 \%$ versus $13 \% ; p=0.042$ ). No significant differences were found between the two patient groups in age, living arrangements, and educational level. Fewer patients with NYHA Class III symptoms attended at least four sessions compared with those who attended less than four sessions (31\% versus $48 \%$; $p=0.044)$. No significant differences were found for health-related quality of life, self-care behaviour, and cognitive status nor for symptoms of anxiety and feelings of depression, self-efficacy expectancies, and perceived control as assessed at baseline. We further determined that NYHA classification and sex were not interrelated.

\section{Discussion}

This evaluation study showed that the programme was considered feasible according to both patients and leaders. Feasibility was based on (1) positive outcomes from the leaders regarding practicability of the CDSMP protocol; (2) positive outcomes regarding patients' attendance and adherence (i.e. overall adherence to the programme and home work assignments) according to both patients and leaders; and (3) positive opinions about the CDSMP from both patients and leaders. Patients and leaders were asked questions about adherence to home work assignments as well as their opinions about the programme. Their answers corresponded largely because both patients and leaders reported positively about action-planning. In addition, their recommendations included points of agreement, i.e., taking additional time for specific topics and improving individual input during these activities as well as taking patients' different characteristics into account.

To gain insight into long-term adherence to the programme and perceived benefits of the programme, we conducted a second evaluation after one year. This evaluation showed that long-term perceived benefits of the programme seemed to diminish to some extent, particularly those benefits representing the components of the programme that patients most appreciated directly after the programme: action-planning and exercise activities (Table 5). In contrast, we found improved percentages for the contribution of the programme to sensible medicine use, following diet instructions, and using cognitive symptom management techniques one year later compared with 
percentages measured directly after the programme. In addition, the patients reported still benefiting relatively much from the programme content after one year (Table 6).

Finally, subgroup analysis revealed that female sex and lower NYHA classification (indicating less severe CHF symptoms) predicted good attendance to the programme. Other socio-demographic, health-related, and psychosocial variables were not related to attendance. It seems plausible that patients with NYHA Class III symptoms may attend fewer sessions because of their marked limitation of physical activity. These results indicate that special attention should be paid to specify the patient-selection criteria for this programme in more detail.

\section{Limitations}

Regarding the original intervention, we made one important adaptation in our choice of who lead the programme: the CDSMP classes in our study were led by teams comprising a cardiac nurse specialist ('professional leader') and a CHF patient ('peer leader'), instead of two trained volunteer lay leaders. CHF patients were included in the team to improve modelling in the CDSMP classes. Including CHF peer leaders may have affected the programme's continuity negatively in terms of attendance to the programme sessions and overall availability for the programme. Compared with volunteer lay leaders, there may be a greater chance for CHF peer leaders to not attend all six sessions of the programme because $\mathrm{CHF}$ has an erratic and unpredictable course, for example, patients have a five-fold increased risk of sudden death. ${ }^{4,5}$ In addition, CHF peer leaders may conduct the programme only for a limited period after being extensively trained in the CDSMP protocol. ${ }^{43}$ Training CHF peer leaders may therefore be less efficient compared with training volunteer lay leaders. Therefore, we included a nurse specialist in the team not only to facilitate implementation of the programme in regular healthcare in The Netherlands but also to positively influence programme continuity. This seemed to be a justified choice: on nine occasions, the peer leader was absent because of illness or other reasons, which affected programme continuity to some extent and therefore was considered a limitation of the study. Furthermore, replacement of the peer leader was difficult because we needed to conduct 21 consecutive CDSMP classes with a limited number of peer leaders. Not replacing an absent peer leader (with exception of the first programme session) was considered a second limitation of the study. Therefore, developing and maintaining a volunteer structure of 
CHF patients to co-lead the CDSMP might be challenging. By including professional leaders to the programme, we improved the continuity of the programme; at no time during the study period was a single session cancelled because of programme leaders unavailability. Furthermore, based on the results of the structured group interview and consistent with previous findings, ${ }^{42}$ both professional and peer leaders had favourable opinions about co-leading the CDSMP. Our findings are in accordance with those of Lorig and colleagues, ${ }^{37}$ who recently reported that even the maintenance of a volunteer structure by using peer leaders was difficult to develop and maintain. Toward the end of their study period, combined professional and peer co-leadership became more common as well. ${ }^{37}$

\section{Recommendations}

Based on the results of this study we can make four recommendations. First, we recommend reconsidering the sequence of the activities in session four 'advance directives for healthcare', 'communication skills', and 'problemsolving' - and simplifying the contents of these activities. In more than half of the classes, participants had difficulties dealing with these topics or even lost motivation to attend these activities. By 2006, some small revisions had already been made to the original CDSMP protocol, ${ }^{44}$ including a sequence revision and content revision of the activities in session four. ${ }^{46}$ Our findings support these modifications.

Second, we recommend extending the time spent on several CDSMP topics and allowing more time for the exchange of personal patients' experiences. Particularly the time spent on activities such as 'medication usage', 'healthy eating', and 'advance directives for healthcare' (e.g. in an end-of-life situation) could be extended to patients' benefit. CHF patients might need to discuss these topics more extensively because the selfmanagement process is challenging for these patients, and their selfmanagement abilities might be poor at the outset. ${ }^{17,47}$ Furthermore, CHF patients are more likely to be facing death than other chronically ill patient groups. In fact, CHF has a higher mortality rate than many cancers and an equivalent symptom burden and severity. ${ }^{48}$ In addition, CHF patients often have poorer understanding of their prognosis and experience difficulties discussing end-of-life issues. ${ }^{49,50}$

Third, we recommend that the target group for CDSMP classes be specified more in detail for CHF patients, for example, to create more homogenous groups of patients. Some patients experienced rather large 
differences between themselves and other patients in the class regarding age and perceived CHF symptoms. Although social comparison seems inevitable, because all participants in our study had the same chronic condition, matching specific patient characteristics, such as age or NYHA classification, might improve patients' exchange of personal experiences. Preferably, subgroups should be defined as to who would benefit most from the CDSMP and who would attend classes more regularly. What might be recommended in specifying the target group is to select on time interval from CHF diagnosis because some patients specifically would have preferred attending the programme earlier after diagnosis $(n=3)$ or were already familiar with most of the content at the time they attended the programme $(n=9)$. While selecting future patients for the programme, special attention should be given to informing, facilitating, and motivating the male participants having more severe CHF symptoms (NYHA Class III) because they showed a relatively low attendance rate in our study. Furthermore, allowing partners of CHF patients to attend the programme might improve patients' attendance. In addition, some patients $(n=6)$ recommended more involvement of partners or family members. Although the CDSMP protocol allows partners or family members to attend the programme, we did not specifically invite them for logistic reasons.

Based on our findings, we do not recommend specifying the content of the intervention for various CHF patient groups (e.g. NYHA classification). More in-depth analyses (Student $t$ test for independent samples) showed that NYHA Class II and III CHF patients did not differ in their perceptions of how much they benefited from the programme ('relatively much' $71.0 \%$ and $77.8 \%$, respectively; $p=0.702$ ); the mean number of action plans made (4.4 and 4.2 , respectively; $p=0.412$ ) and accomplished per patient (3.8 and 3.7, respectively; $p=0.649$ ) per patient; and mean report mark for the programme (8.1 and 8.0, respectively; $p=0.609$ ). Furthermore, the original CDSMP was designed as a generic programme and is based on self-tailoring, e.g., through personal action-planning. ${ }^{8}$

Our last recommendation refers to adding a booster session to the CDSMP six or twelve months after attending the programme because we found that the perceived benefits of the programme regarding action-planning and exercising diminished somewhat in the long run. Furthermore, immediately after the programme, four patients recommended adding booster sessions to the programme. In addition, at the second evaluation, more than half of the patients reported they would have preferred a booster session at six or twelve months after attending the class. Patients also mentioned that they had not frequently used the CDSMP reference book during the year after 
the programme and that only a few of them had kept contact with class members. In this booster session, daily experiences from the previous one year period could be exchanged; some of the main self-management techniques could be practiced again (e.g. action-planning and cognitive symptom management techniques); and new exercise goals and action plans could be made. However, future research is needed to study whether adding such a booster session would increase effectiveness of the programme in the long term (i.e. one year after the class).

\section{Comments}

This study reported on the feasibility of the CDSMP ${ }^{8,29-31}$ among CHF patients with NYHA Class II and III symptoms. The programme emphasizes patients' central role and responsibility in managing their own illness and teaches patients to become active self-managers, which is crucial for CHF selfmanagement. Based on the results of our study, this comprehensive groupbased programme seems applicable to a homogeneous patient group of $\mathrm{CHF}$ patients with NYHA Class II to III symptoms in The Netherlands. Future research could focus on the exploring the added value of a booster session and assessing determinants of attendance and adherence to the programme, e.g. including more time for patient exchange of personal experiences, by specifying the group of patients to which the programme is targeted more in detail, or by allowing partners of $\mathrm{CHF}$ patients to attend the programme as well.

\section{Acknowledgements}

This research project was funded by the Netherlands Heart Foundation (2002B005) and the University Hospital Maastricht (PF 179), The Netherlands. This study was conducted within CAPHRI School for Public Health and Primary Care of Maastricht University in The Netherlands in cooperation with Lorig and colleagues from Stanford University. We thank N. Steverink, H.A. Elzen, and J.P. Slaets from the University Medical Center Groningen in The Netherlands for their willingness to share Dutch intervention materials. 


\section{References}

1. Clark RA, McLennan S, Dawson A, Wilkinson D, Stewart S. Uncovering a hidden epidemic: a study of the current burden of heart failure in Australia. Heart Lung Circ 2004;13(3):266-73.

2. Stewart S, Maclntyre K, Capewell S, McMurray JJ. Heart failure and the aging population: an increasing burden in the 21st century? Heart 2003;89(1):49-53.

3. Thomas S, Rich MW. Epidemiology, pathophysiology, and prognosis of heart failure in the elderly. Clin Geriatr Med 2007;23(1):1-10.

4. van Jaarsveld $\mathrm{CH}$, Ranchor AV, Kempen Gl, Coyne JC, van Veldhuisen DJ, Sanderman R. Epidemiology of heart failure in a community-based study of subjects aged $>$ or $=57$ years: incidence and long-term survival. Eur $J$ Heart Fail 2006;8(1):23-30.

5. Mosterd A, Cost B, Hoes AW, de Bruijne MC, Deckers JW, Hofman A, et al. The prognosis of heart failure in the general population: The Rotterdam Study. Eur Heart J 2001;22(15):1318-27.

6. Heo S, Moser DK, Lennie TA, Zambroski CH, Chung ML. A comparison of healthrelated quality of life between older adults with heart failure and healthy older adults. Heart Lung 2007;36(1):16-24.

7. Creer TL, Renne CM, Christian WP. Behavioral contributions to rehabilitation and childhood asthma. Rehabil Lit 1976;37(8):226-247.

8. Lorig KR, Holman H. Self-management education: history, definition, outcomes, and mechanisms. Ann Behav Med 2003;26(1):1-7.

9. Riegel B, Dickson VV. A situation-specific theory of heart failure self-care. $J$ Cardiovasc Nurs 2008;23(3):190-6.

10. Moser DK, Watkins JF. Conceptualizing self-care in heart failure: a life course model of patient characteristics. J Cardiovasc Nurs 2008;23(3):205-218.

11. Deaton C. Outcome measurement: self-management in heart failure. $J$ Cardiovasc Nurs 2000;14(4):116-8.

12. Jaarsma T, Stromberg A, Martensson J, Dracup K. Development and testing of the European Heart Failure Self-Care Behaviour Scale. Eur $J$ Heart Fail 2003;5(3):363-70.

13. Belardinelli R, Georgiou D, Cianci G, Purcaro A. Randomized, controlled trial of long-term moderate exercise training in chronic heart failure: effects on functional capacity, quality of life, and clinical outcome. Circulation 1999;99(9):1173-82.

14. Lorig K, Holman H, Sobel D, Laurent D, Gonzalez V, Minor M. Living a healthy life with chronic conditions: self-management of heart disease, arthritis, diabetes, asthma, bronchitis, emphysema and others. 2nd ed. Boulder, Colorado: Bull Publishing Company; 2000.

15. Evangelista LS, Shinnick MA. What do we know about adherence and self-care? J Cardiovasc Nurs 2008;23(3):250-7.

16. van der $\mathrm{Wal} \mathrm{MH}$, Jaarsma $\mathrm{T}$. Adherence in heart failure in the elderly: Problem and possible solutions. Int J Cardiol 2008;125(2):203-8.

17. Riegel B, Carlson B. Facilitators and barriers to heart failure self-care. Patient Educ Couns 2002;46(4):287-95. 
18. Scott LD, Setter-Kline K, Britton AS. The effects of nursing interventions to enhance mental health and quality of life among individuals with heart failure. Appl Nurs Res 2004;17(4):248-56.

19. Harrison MB, Browne GB, Roberts J, Tugwell P, Gafni A, Graham ID. Quality of life of individuals with heart failure: a randomized trial of the effectiveness of two models of hospital-to-home transition. Med Care 2002;40(4):271-82.

20. GESICA Investigators. Randomised trial of telephone intervention in chronic heart failure: DIAL trial. BMJ 2005;331(7514):425.

21. Ojeda S, Anguita M, Delgado M, Atienza F, Rus C, Granados AL, et al. Short- and long-term results of a programme for the prevention of readmissions and mortality in patients with heart failure: are effects maintained after stopping the programme? Eur J Heart Fail 2005;7(5):921-6.

22. Gwadry-Sridhar FH, Arnold JM, Zhang Y, Brown JE, Marchiori G, Guyatt G. Pilot study to determine the impact of a multidisciplinary educational intervention in patients hospitalized with heart failure. Am Heart J 2005;150(5):982.

23. de la Porte PW, Lok DJ, van Veldhuisen DJ, van Wijngaarden J, Cornel JH, Zuithoff NP, et al. Added value of a physician-and-nurse-directed heart failure clinic: results from the Deventer-Alkmaar heart failure study. Heart 2007;93(7):819-25.

24. Shively M, Kodiath M, Smith TL, Kelly A, Bone P, Fetterly L, et al. Effect of behavioral management on quality of life in mild heart failure: a randomized controlled trial. Patient Educ Couns 2005;58(1):27-34.

25. Doughty RN, Wright SP, Pearl A, Walsh HJ, Muncaster S, Whalley GA, et al. Randomized, controlled trial of integrated heart failure management: The Auckland Heart Failure Management Study. Eur Heart J 2002;23(2):139-46.

26. Austin J, Williams R, Ross L, Moseley L, Hutchison S. Randomised controlled trial of cardiac rehabilitation in elderly patients with heart failure. Eur $J$ Heart Fail 2005;7(3):411-7.

27. Moser DK. Psychosocial factors and their association with clinical outcomes in patients with heart failure: why clinicians do not seem to care. Eur $J$ Cardiovasc Nurs 2002;1(3):183-8.

28. Konstam V, Moser DK, De Jong MJ. Depression and anxiety in heart failure. $J$ Card Fail 2005;11(6):455-63.

29. Lorig KR, Sobel DS, Stewart AL, Brown BW, Jr., Bandura A, Ritter P, et al. Evidence suggesting that a chronic disease self-management program can improve health status while reducing hospitalization: a randomized trial. Med Care 1999;37(1):5-14.

30. Lorig KR, Ritter P, Stewart AL, Sobel DS, Brown BW, Jr., Bandura A, et al. Chronic disease self-management program: 2-year health status and health care utilization outcomes. Med Care 2001;39(11):1217-23.

31. Lorig KR, Sobel DS, Ritter PL, Laurent D, Hobbs M. Effect of a self-management program on patients with chronic disease. Eff Clin Pract 2001;4(6):256-62.

32. Bandura A. Self-efficacy: the exercise of control. New York: Freeman; 1997.

33. Swerissen H, Belfrage J, Weeks A, Jordan L, Walker C, Furler J, et al. A randomised control trial of a self-management program for people with a chronic illness from Vietnamese, Chinese, Italian and Greek backgrounds. Patient Educ Couns 2006;64(1-3):360-8. 
34. Siu AM, Chan CC, Poon PK, Chui DY, Chan SC. Evaluation of the chronic disease self-management program in a Chinese population. Patient Educ Couns 2007;65(1):42-50.

35. Lorig KR, Ritter PL, Jacquez A. Outcomes of border health Spanish/English chronic disease self-management programs. Diabetes Educ 2005;31(3):401-9.

36. Lorig KR, Ritter PL, Gonzalez VM. Hispanic chronic disease self-management: a randomized community-based outcome trial. Nurs Res 2003;52(6):361-9.

37. Lorig KR, Hurwicz ML, Sobel D, Hobbs M, Ritter PL. A national dissemination of an evidence-based self-management program: a process evaluation study. Patient Educ Couns 2005;59(1):69-79.

38. Fu D, Fu H, McGowan P, Shen YE, Zhu L, Yang $H$, et al. Implementation and quantitative evaluation of chronic disease self-management programme in Shanghai, China: randomized controlled trial. Bull World Health Organ 2003;81(3):174-82.

39. Chan SC, Siu AM, Poon PK, Chan CC. Chronic disease self-management program for Chinese patients: a preliminary multi-baseline study. Int $J$ Rehabil Res 2005;28(4):351-4.

40. Kempen GI, Sanderman R, Miedema I, Meyboom-de Jong B, Ormel J. Functional decline after congestive heart failure and acute myocardial infarction and the impact of psychological attributes. A prospective study. Qual Life Res 2000;9(4):439-50.

41. Wu JR, Moser DK, Lennie TA, Burkhart PV. Medication adherence in patients who have heart failure: a review of the literature. Nursing Clinics of North America 2008;43(1):133-53.

42. Smeulders ES, van Haastregt JC, Dijkman-Domanska BK, van Hoef EF, van Eijk JT, Kempen GI. Nurse- and peer-led self-management programme for patients with an implantable cardioverter defibrillator; a feasibility study. BMC Nurs 2007;6:6.

43. Smeulders ES, van Haastregt JC, van Hoef EF, van Eijk JT, Kempen GI. Evaluation of a self-management programme for congestive heart failure patients: design of a randomised controlled trial. BMC Health Serv Res 2006;6:91.

44. Lorig K, Gonzalez V, Laurent D. The chronic disease self-management program master trainer's guide. Palo Alto, CA: Stanford University; 1999.

45. Elzen H, Slaets JP, Snijders TA, Steverink N. Evaluation of the chronic disease self-management program (CDSMP) among chronically ill older people in the Netherlands. Soc Sci Med 2007;64(9):1832-41.

46. Lorig K, Gonzalez V, Laurent D. The chronic disease self-management program master trainer's manual. Palo Alto, CA: Stanford University; 2006.

47. Carlson B, Riegel B, Moser DK. Self-care abilities of patients with heart failure. Heart Lung 2001;30(5):351-9.

48. Thompson DR. Improving end-of-life care for patients with chronic heart failure. Heart 2007;93(8):901-2.

49. Murray SA, Boyd K, Kendall M, Worth A, Benton TF, Clausen H. Dying of lung cancer or cardiac failure: prospective qualitative interview study of patients and their carers in the community. BMJ 2002;325(7370):929. 
50. Selman L, Harding R, Beynon T, Hodson F, Coady E, Hazeldine C, et al. Improving end-of-life care for patients with chronic heart failure: "Let's hope it'll get better, when I know in my heart of hearts it won't". Heart 2007;93(8):963-7. 
Feasibility study 



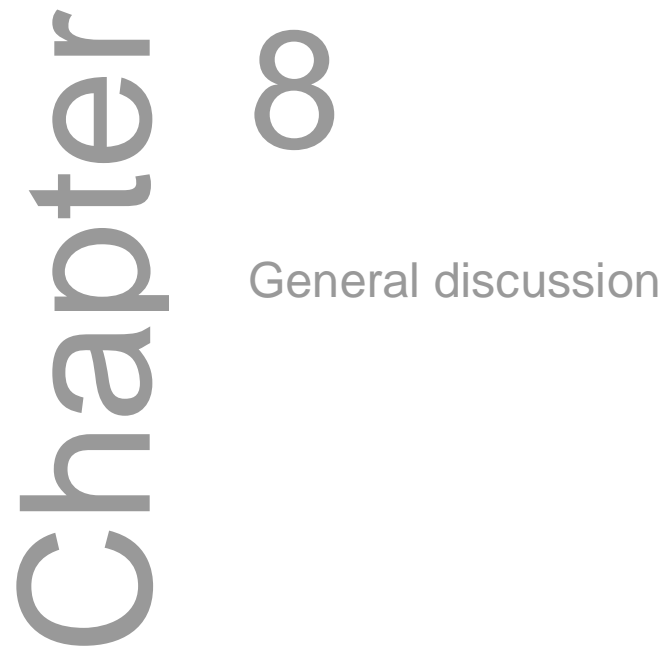




\section{Introduction}

Congestive heart failure (CHF) has a serious impact on patients' physical, social and emotional functioning and for most patients adhering to the complex treatment protocol in order to prevent CHF exacerbations and hospital readmissions is a challenge.$^{1-4}$ In previous years, various intervention programmes have been developed to improve self-management abilities among CHF patients, mostly by providing education about treatment adherence. ${ }^{5,6} \mathrm{CHF}$ nurse support focuses mainly on these medical aspects as well. ${ }^{7-9}$ Although crucial, CHF self-management exceeds simply managing the medical aspects of the disease as patients also face considerable difficulties dealing with the social and emotional consequences of CHF. Patients can no longer fulfil the demands of their social life or they suffer from psychological distress, which affects treatment adherence and quality of life. ${ }^{10-13}$ The Chronic Disease Self-Management Programme (CDSMP) aims to improve patients' medical, social and emotional skills for managing a chronic condition. ${ }^{14-17}$ The CDSMP is a widely disseminated and evaluated programme, applied to patients with different chronic conditions. ${ }^{14-16,18-40}$ Yet, the effectiveness and feasibility of the programme solely among CHF patients remains to be shown. For this reason, the effectiveness and feasibility of the CDSMP among CHF outpatients in The Netherlands were assessed in a twogroup randomized controlled trial with twelve months of follow-up after start of the programme. The two objectives of this thesis were:

(1) to assess the effects of the CDSMP on psychosocial attributes, health behaviour, quality of life, and healthcare utilization among CHF patients; and

(2) to assess the feasibility of the CDSMP in terms of (a) performance of the intervention according to protocol, (b) patients' adherence to the intervention, and (c) the opinions of the patients and leaders about the intervention.

First, an overview of the main findings of our study is provided. Second, the main findings of the study are discussed in light of previous findings. Third, methodological considerations are addressed. Finally, implications for practice and future research are provided, as well as a general conclusion.

\section{Main findings}

Programme effectiveness among CHF patients who experienced a slight to marked limitation of physical activity was studied for psychosocial attributes, health behaviour, quality of life and healthcare utilization (Chapters 4 and 5). 
Regarding psychosocial attributes, the programme significantly improved patients' use of cognitive symptom management techniques directly after attending the programme. No favourable effects were found for self-efficacy expectancies and perceived control. Short-term improvements on health behaviour were found for self-care behaviour and physical activity (i.e. walking for exercise and other physical activities such as aerobic, stretching and strength exercises, sports, and gardening). The favourable effect on other physical activities lasted until six months of follow-up after start of the programme. The programme did not affect smoking behaviour, drinking behaviour and body mass. Intervention group patients significantly improved their cardiac-specific quality of life directly after the programme. No effects were observed for (general) health-related quality of life, perceived autonomy, symptoms of anxiety, or feelings of depression. The CDSMP did not affect patients' healthcare utilization. In summary, most of the effects were shortterm and did not sustain until six or twelve months of follow-up after start of the programme.

Additional subgroup analyses were conducted to assess which patients benefited most from attending the CDSMP with respect to cardiac-specific quality of life (Chapter 6). These analyses revealed that patients with better cognitive status benefited more from the CDSMP than their poorer functioning counterparts in the short term. No long-term difference in cardiac-specific quality of life was found between the two subgroups, however. In addition, patients who attended at most lower secondary (vocational) education benefited significantly more from the CDSMP than their higher educated counterparts during the total follow-up period. No subgroup effects were found for disease-related patient characteristics (i.e. time interval from $\mathrm{CHF}$ diagnosis, New York Heart Association (NYHA) classification, and number of active comorbid diseases), or other socio-demographic patient characteristics (i.e. sex, age, and living arrangements).

Feasibility of the programme was initially assessed in a small-scale pilot study of ten patients with an implantable cardioverter defibrillator (ICD) before a more comprehensive feasibility study alongside the trial among CHF patients (Chapters 2 and 7). This feasibility study showed that $80 \%$ of the programme sessions were carried out according to protocol. Three-quarters of the patients attended at least four of the six CDSMP programme sessions. Furthermore, most patients made weekly action plans which they accomplished on a weekly basis. After attending the programme, a large proportion of patients perceived themselves as able to exercise more (81\%) and use action plans to achieve personal goals (82\%). After one year of follow-up these proportions were $69 \%$ and $58 \%$, respectively. Both patients 
and leaders recommended taking additional time for discussing specific topics as well as taking differences in patient characteristics into account. Females and those with lower NYHA classifications (indicating better functioning) predicted good attendance to the programme.

To conclude, the CDSMP significantly improved patients' use of cognitive symptom management techniques, self-care behaviour, physical activity levels, and cardiac-specific quality of life directly after the intervention, but most of these effects diminished at six and twelve months after the start of the programme. Patients and leaders considered the programme feasible. After attending the programme, a large number of patients perceived themselves able to make behavioural changes, however, in the long term this decreased considerably.

\section{Possible explanations of moderate effects}

In this section, we discuss possible explanations for the moderate and shortterm effects of the programme.

\section{Contrast between the programme and regular care}

One explanation for the moderate effects is that the contrast between the CDSMP and regular care in The Netherlands is too small to detect substantial additional effects of the programme among CHF patients. The CDSMP has been evaluated in many different healthcare settings worldwide, showing inconsistencies in programme effectiveness throughout those studies. ${ }^{14-16,18-40}$ This may indicate that the effectiveness of the CDSMP depends on the organization of the healthcare setting in which the programme is implemented. In The Netherlands, Elzen and colleagues ${ }^{30,36}$ evaluated the CDSMP among a heterogeneous group of chronically-ill patients $(n=144)$. No favourable effects of the programme were found compared with regular care. Among CHF patients, the CDSMP was only effective in the short term (Chapters 4 and 5). These results raise the question of whether we can expect the CDSMP to make a large impact in a healthcare setting such as The Netherlands.

Programme effectiveness may also largely depend on the level of regular care for specific CHF. The level and accessibility of regular CHF care in The Netherlands are considered rather high. Most Dutch hospitals, for example, provide a CHF management programme for outpatients. ${ }^{41}$ Furthermore, most 
standard programmes incorporate exercise and behavioural interventions. Therefore, elements of regular CHF care are already part of the CDSMP. This may have diminished the contrast between the intervention and control groups in detecting substantial favourable effects of the programme.

\section{Duration of the programme}

Another explanation of the moderate effects may be that the programme is too short to achieve long-term behavioural change in CHF patients. The CDSMP motivates patients to set personal goals and make weekly action plans to achieve behavioural changes. During the six weeks of the programme, patients practice making action plans. However, it is questionable whether six times is enough to incorporate the processes of goal-setting, action-planning and problem-solving into daily life. In addition, participation in these activities is voluntary, meaning patients may practice these skills even less. Finally, the content of these action plans does not need to be diseaserelated, so the gain in perceived health may be limited as well. The behavioural management programme by Shively and colleagues ${ }^{42}$ is comparable with the CDSMP as both programmes enhance behavioural changes by establishing specific goals. However, the programme by Shively and colleagues ${ }^{42}$ is spread over four months and consists of four two-hour group sessions and three additional phone calls. During the telephone calls, questions are clarified, patients' activity levels are adjusted if desirable and new goals are set. ${ }^{42}$ The programme was tested in a randomized controlled trial $(n=116)$ and significantly improved the physical dimension of cardiacspecific quality of life over time. The emotional dimension was unaffected by the programme, however. In addition, no effect was found for general quality of life, ${ }^{42}$ which is consistent with the current study (Chapter 4). Nevertheless, the study by Shively and colleagues ${ }^{42}$ provides leads for future research to improve the CDSMP in CHF patients, such as extending the programme over a longer time period and adding motivational telephone calls by nurses to support behavioural change.

\section{Generic programme versus homogeneous patient group}

The moderate effects may also be explained by the fact that the CDSMP is a generic programme developed for use among heterogeneous groups of chronically ill people. The format of the CDSMP is possibly too generic to 
improve (long-term) outcomes among CHF patients because CHF, in contrast to most chronic conditions, is a progressive disorder with a poor prognosis. ${ }^{2,43-45}$ In addition, poor medical self-management among CHF patients may lead directly to serious consequences in terms of acute exacerbations and functional deterioration, ${ }^{46}$ which may be less the case for other chronic conditions. In the past few years, several CDSMP evaluation studies have been conducted among homogeneous groups of patients, most showing limited effects. ${ }^{25,32,34,39}$ In the study by Haas and colleagues, ${ }^{25}$ the CDSMP showed little to no advantage for most of the outcomes among patients with chronic low back pain $(n=109)$. In the study by Kendall and colleagues ${ }^{32}$ among patients with stroke $(n=100)$, no significant effects were found, with the exception of self-care at nine months of follow-up. In a four-group randomized controlled trial among patients with chronic depression $(n=104)$, the CDSMP was supplemented with ongoing bi-monthly groups focused on continued goal-setting and problem-solving, but no statistically significant differences could be detected. ${ }^{34}$ Finally, a recent study among myocardial infarction patients $(n=192)$ showed no statistically significant differences between the two groups on outcomes such as self-efficacy and health behaviours. ${ }^{39}$ These limited findings raise the question of whether promising results among specific patient groups can be expected from a generic selfmanagement programme.

Recently, efforts have been made to improve applicability of the programme by adapting the CDSMP to specific conditions, such as chronic stable angina ${ }^{37}$ and chronic obstructive pulmonary disease (http:// www.inse.qmul.ac.uk/chsgppc/bella/index.html). The study among patients with chronic stable angina showed favourable results immediately after the end of the programme on quality of life and self-efficacy, however, no longterm follow-up was included. ${ }^{37}$ The pilot study among patients with chronic obstructive pulmonary disease is still ongoing. Therefore, it is not yet possible to conclude that adapting the CDSMP to specific chronic conditions improves effectiveness. Besides, previous research in which the relative effectiveness of a generic self-management programme (CDSMP) was compared with a disease-specific self-management programme (Arthritis Self-Management Programme (ASMP)) showed no differences among arthritis patients in positive effects between both programmes. ${ }^{26,31}$ 


\section{Selection of target population}

Finally, the moderate effects of the programme may be influenced by the method of selecting our target population. In our study, patients were selected based on a CHF diagnosis and perceived CHF symptoms. After consulting with several cardiologists, we decided to include CHF patients with a slight to marked limitation of physical activity (corresponding with NYHA Classes II and III) as these patients were considered to have specific management problems caused by the severity of the disease. By excluding CHF patients in NYHA Classes I and IV, we omitted patients without substantial symptoms and disease-related management problems and patients with severe symptoms unable to attend group sessions. Besides NYHA classification, no other selection criteria were set, although it is possible that specific subgroups of $\mathrm{CHF}$ patients may benefit more than the group of patients selected from the programme. However, in additional subgroup analyses, contrary to what we expected no differential effects of the programme were found for age, time interval from CHF diagnosis, and NYHA classification (Chapter 6). On the other hand, subgroup analyses showed that CHF patients with better cognitive status and patients with lower educational levels benefited most from the CDSMP in terms of cardiac-specific quality of life (Chapter 6). This indicates that programme effectiveness may be improved by aiming the programme at specific subgroups of CHF patients.

\section{Methodological considerations}

Some methodological considerations for interpreting the outcomes of this study merit attention.

First, in our study, cardiologists and CHF nurse specialists recruited patients while they visited the heart failure/cardiology outpatient clinic. According to the inclusion procedure, cardiologists and CHF nurse specialists had to inform all eligible patients about the study. It is unclear, though, whether they actually have informed all eligible patients. However, all participating cardiologists and CHF nurse specialists were well instructed about the inclusion procedure at the start of the study. In addition, the researchers facilitated the selection process by pre-screening eligible patients from the computer system and adding reminders to check study eligibility on patients' files. Therefore, it seems unlikely that we systematically missed eligible patients. On the other hand, no complete assessment could be conducted on the representativeness of the study sample as background 
information (sex, age, and NYHA classification) was not obtained from all eligible patients $(n=717)$.

Second, patient-selection processes during the randomized controlled trial may have threatened the internal validity of our study. A considerable share of the participants $(21.5 \%, n=40)$ did not start the programme or decided to stop within the first two sessions. Patients who stopped or refused participation in the self-management programme were approached for followup measurements and included in the intention-to-treat analyses. Regarding attrition during the follow-up measurements, 265 patients (83.6\%) completed the trial (baseline measurement plus at least the last follow-up measurement) and 52 patients were lost to follow-up. The attrition was not considered selective, as the proportions of, and reasons for, drop-out were similar in the intervention and control groups (Chapters 4-6).

A third consideration to be addressed is the randomization procedure in our trial. Our trial consisted of eleven consecutive cycles in which patients were randomly allocated to the intervention or control group after completing the baseline measurement. Despite random allocation, patients in the intervention group reported significantly lower cardiac-specific quality of life at baseline than control group patients. No differences in other baseline characteristics were found between the groups. The significant difference in baseline cardiac-specific quality of life may have influenced the positive shortterm effect on cardiac-specific quality of life as patients in the intervention group possibly had more to gain from the programme based on these lower baseline levels. Therefore, we adjusted for the baseline level of cardiacspecific quality of life in all analyses to minimise the potential bias of this significant baseline difference (Chapters 4 and 6).

Finally, the possible contamination of control group patients merits attention. In our study, cardiac nurse specialists conducted the CDSMP classes in combination with their usual work in the hospital. Control patients may have been affected by the CDSMP content during outpatient visits if they were checked by a trained cardiac nurse specialist. At most, contamination may have occurred in three of the six hospitals, as four hospitals provided trained nurse specialists but the nurse specialist from the fourth hospital was temporarily not working at the outpatient clinic during the study period. Furthermore, to reduce the chance of contamination of the control group, we explicitly stressed the importance for nurse specialists to distinguish their CDSMP leadership from their usual work in the hospital. During the study, they were asked not to speak about the intervention programme with patients at the outpatient clinic, not even with patients who participated in the 
programme. Possible contamination of the control group was not checked afterwards, however, so its exact impact in our study remains unclear.

\section{Implications for practice and future research}

The worldwide popularity of the CDSMP reflects the insight that chronically ill patients urgently need self-management support to improve their selfmanagement abilities, daily functioning, and quality of life. While large randomized controlled trials have shown inconsistent effects of the programme on outcomes such as health status, self-efficacy, health behaviour, and healthcare utilization, many CDSMP participants have given powerful accounts of their benefits. Perhaps this explains why the UK government has invested substantially in the anglicised version of the CDSMP (Expert Patient Programme (EPP)) since 2001 - in the face of limited evidence - by implementing the programme nationwide with the goal of providing it to 100,000 chronically ill patients by $2012 .{ }^{47}$ So, what are the implications for practice and future research based on our study on $\mathrm{CHF}$ patients in The Netherlands?

\section{Implications for practice}

Based on the fact that the CDSMP had only limited effects on patients' outcomes, including the current form of the CDSMP into the regular care of $\mathrm{CHF}$ patients in The Netherlands is not recommended. We recommend at least two adaptations to improve the effectiveness of the programme.

First, the programme format may be extended by two to four sessions. By adding a number of sessions participants can practice goal-setting, actionplanning and problem-solving over a longer period of time, which may improve the incorporation of action-planning in their daily lives in the longer term. In addition, more time could be spent on specific programme topics that overran their allotted time in the current protocol because participants considered these topics difficult to comprehend or focus on (Chapter 7).

Second, to accomplish long-term behavioural change among $\mathrm{CHF}$ patients, the CDSMP may have to be extended to include nurse- and peer-led booster group sessions and/or nurse-led individual follow-up contacts after the end of the programme. Booster group sessions (after, for example, six months) would be useful for exchanging personal experiences from the preceding months. In addition, the main self-management skills could be 
reinforced, for example, the processes of goal-setting, action-planning and problem-solving, and cognitive symptom management techniques (e.g. breathing techniques and muscle relaxation). Finally, new (exercise) goals and action plans could be made. Individual telephone-based and/or outpatient follow-ups may be particularly useful for coaching patients to integrate physical activity into their daily routines, support long-term adherence to lifestyle changes, and address patients' psychosocial challenges in adapting to CHF. Supporting patients' self-management behaviour (including providing psychosocial care on managing CHF) could also be integrated into a patient's regular contact with their cardiac nurse specialist in order to improve implementation of these CDSMP elements into regular $\mathrm{CHF}$ care.

In addition to the suggested adaptations for improving programme effectiveness, we also have some suggestions on improving the programme's continuity. It is important that the availability of both trained leaders and newly recruited participants is guaranteed in the future. A necessary condition for nurse specialists to conduct CDSMP classes is that nurse support on patients' medical, social, and emotional self-management behaviour is embedded in regular CHF care. Furthermore, a sufficiently large group of peer leaders need to be trained to guarantee continuity of the programme and prevent peer leaders becoming overloaded. Peer leaders could be recruited from previous CDSMP classes, which would have the advantage that patients are already familiar with the content of the programme. Extra training could then be given on modelling, group dynamics, and supporting patients' action-planning. Regarding patient recruitment, it is important that nurse specialists are offered the opportunity to invest time in selecting new patients for the programme.

\section{Implications for future research}

Based on our study findings, a number of recommendations for future research on the CDSMP among CHF patients can be made.

First, in order to assess the added value of adaptations such as extending the programme and including follow-up contacts, a pilot study should be performed to assess the feasibility of the adapted programme and gain insight into its potential effectiveness. If the results of this pilot study are promising, we recommend subsequently performing a randomized controlled trial to assess the (cost) effectiveness of the new programme.

Second, cardiac-specific self-efficacy (as a central explaining concept of effectiveness) may be measured differently in future research. In this study, four items from the Cardiac Self-Efficacy Questionnaire ${ }^{48}$ were excluded 
because of missing values. Although self-efficacy is considered the key mechanism of the programme, it was not significantly improved among CHF patients. As this finding may have been affected by our choice of measure, the working mechanism of the programme among CHF patients needs to be studied more thoroughly. Such knowledge may further improve the programme.

Third, in this study, explorative subgroup analyses were conducted, which indicated that a lower educational level is a significant predictor for the effectiveness of the CDSMP on cardiac-specific quality of life among CHF patients. However, future research is necessary to confirm the influence of educational level on the effectiveness of the CDSMP among CHF patients. In addition, lower and higher educated patients may have different experiences of goal-setting and action-planning from their educational and working backgrounds. Future research may further explore if tailoring the processes of goal-setting and action-planning to the individual capabilities of lower and, in specific, higher educated patients may improve the programme's effectiveness in both groups.

Finally, a systematic review on the effectiveness of the CDSMP is warranted. In previous years, the programme has been tested in numerous countries and healthcare settings and among different heterogeneous and homogeneous patient samples. As most previous studies showed inconsistent findings, it is important to gain an overview of the results about programme effectiveness to explore possible explanations for these inconsistencies.

\section{General conclusion}

In CHF self-management research, insufficient light has been shed on the psychosocial aspects of patients' self-management. In this thesis, the effectiveness and feasibility of a cognitive-behavioural self-management group programme (i.e. the CDSMP) among CHF patients was studied. The CDSMP emphasizes both medical, social, and emotional self-management and teaches patients to manage their chronic health problems on all three levels. The CDSMP was considered feasible and significantly improved patients' use of cognitive symptom management techniques, self-care behaviour, physical activity levels and cardiac-specific quality of life directly after the end of the programme. However, most of these effects diminished in the longer term. Given these results, the implementation of the CDSMP in Dutch CHF regular care in its current form is not recommended. The 
programme must be adapted and the feasibility and potential effectiveness of these adaptations assessed in future research. 


\section{References}

1. Evangelista LS, Shinnick MA. What do we know about adherence and self-care? $J$ Cardiovasc Nurs 2008;23(3):250-7.

2. Johansson $P$, Dahlstrom $U$, Brostrom $A$. Factors and interventions influencing health-related quality of life in patients with heart failure: a review of the literature. Eur J Cardiovasc Nurs 2006;5(1):5-15.

3. Riegel B, Carlson B. Facilitators and barriers to heart failure self-care. Patient Educ Couns 2002;46(4):287-95.

4. van Jaarsveld $\mathrm{CH}$, Sanderman R, Miedema I, Ranchor AV, Kempen GI. Changes in health-related quality of life in older patients with acute myocardial infarction or congestive heart failure: a prospective study. J Am Geriatr Soc 2001;49(8):10528.

5. Grady KL. Self-care and quality of life outcomes in heart failure patients. $J$ Cardiovasc Nurs 2008;23(3):285-92.

6. Jovicic A, Holroyd-Leduc JM, Straus SE. Effects of self-management intervention on health outcomes of patients with heart failure: a systematic review of randomized controlled trials. BMC Cardiovasc Disord 2006;6:43.

7. Bruggink-Andre de la Porte PW, Lok DJ, van Wijngaarden J, Cornel JH, PruijsersLamers D, van Veldhuisen DJ, et al. Heart failure programmes in countries with a primary care-based health care system. Are additional trials necessary? Design of the DEAL-HF study. Eur J Heart Fail 2005;7(5):910-20.

8. Jaarsma T, Van Der Wal MH, Hogenhuis J, Lesman I, Luttik ML, Veeger NJ, et al. Design and methodology of the $\mathrm{COACH}$ study: a multicenter randomised Coordinating study evaluating Outcomes of Advising and Counselling in Heart failure. Eur J Heart Fail 2004;6(2):227-33.

9. Jaarsma T, van der Wal MH, Lesman-Leegte I, Luttik ML, Hogenhuis J, Veeger $\mathrm{NJ}$, et al. Effect of moderate or intensive disease management program on outcome in patients with heart failure: Coordinating Study Evaluating Outcomes of Advising and Counseling in Heart Failure (COACH). Arch Intern Med 2008;168(3):316-24.

10. Evangelista LS, Kagawa-Singer M, Dracup K. Gender differences in health perceptions and meaning in persons living with heart failure. Heart Lung 2001;30(3):167-76.

11. Konstam V, Moser DK, De Jong MJ. Depression and anxiety in heart failure. $J$ Card Fail 2005;11(6):455-63.

12. Moser DK, Worster PL. Effect of psychosocial factors on physiologic outcomes in patients with heart failure. J Cardiovasc Nurs 2000;14(4):106-15.

13. Wu JR, Moser DK, Lennie TA, Burkhart PV. Medication adherence in patients who have heart failure: a review of the literature. Nursing Clinics of North America 2008;43(1):133-53.

14. Lorig KR, Sobel DS, Stewart AL, Brown BW, Jr., Bandura A, Ritter P, et al. Evidence suggesting that a chronic disease self-management program can improve health status while reducing hospitalization: a randomized trial. Med Care 1999;37(1):5-14. 
15. Lorig KR, Ritter P, Stewart AL, Sobel DS, Brown BW, Jr., Bandura A, et al. Chronic disease self-management program: 2-year health status and health care utilization outcomes. Med Care 2001;39(11):1217-23.

16. Lorig KR, Sobel DS, Ritter PL, Laurent D, Hobbs M. Effect of a self-management program on patients with chronic disease. Eff Clin Pract 2001;4(6):256-62.

17. Lorig KR, Holman H. Self-management education: history, definition, outcomes, and mechanisms. Ann Behav Med 2003;26(1):1-7.

18. Fu D, Fu H, McGowan P, Shen YE, Zhu L, Yang H, et al. Implementation and quantitative evaluation of chronic disease self-management programme in Shanghai, China: randomized controlled trial. Bull World Health Organ 2003;81(3):174-82.

19. Lorig KR, Ritter PL, Gonzalez VM. Hispanic chronic disease self-management: a randomized community-based outcome trial. Nurs Res 2003;52(6):361-9.

20. Wright CC, Barlow JH, Turner AP, Bancroft GV. Self-management training for people with chronic disease: an exploratory study. Br J Health Psychol 2003;8(Pt 4):465-76.

21. Farrell K, Wicks MN, Martin JC. Chronic disease self-management improved with enhanced self-efficacy. Clin Nurs Res 2004;13(4):289-308.

22. Barlow JH, Wright CC, Turner AP, Bancroft GV. A 12-month follow-up study of self-management training for people with chronic disease: are changes maintained over time? Br J Health Psychol 2005;10(Pt 4):589-99.

23. Chan SC, Siu AM, Poon PK, Chan CC. Chronic disease self-management program for Chinese patients: a preliminary multi-baseline study. Int $J$ Rehabil Res 2005;28(4):351-4.

24. Griffiths C, Motlib J, Azad A, Ramsay J, Eldridge S, Feder G, et al. Randomised controlled trial of a lay-led self-management programme for Bangladeshi patients with chronic disease. Br J Gen Pract 2005;55(520):831-7.

25. Haas M, Groupp E, Muench J, Kraemer D, Brummel-Smith K, Sharma R, et al. Chronic disease self-management program for low back pain in the elderly. $J$ Manipulative Physiol Ther 2005;28(4):228-37.

26. Lorig K, Ritter PL, Plant K. A disease-specific self-help program compared with a generalized chronic disease self-help program for arthritis patients. Arthritis Rheum 2005;53(6):950-7.

27. Lorig KR, Hurwicz ML, Sobel D, Hobbs M, Ritter PL. A national dissemination of an evidence-based self-management program: a process evaluation study. Patient Educ Couns 2005;59(1):69-79.

28. Lorig KR, Ritter PL, Jacquez A. Outcomes of border health Spanish/English chronic disease self-management programs. Diabetes Educ 2005;31(3):401-9.

29. Swerissen H, Belfrage J, Weeks A, Jordan L, Walker C, Furler J, et al. A randomised control trial of a self-management program for people with a chronic illness from Vietnamese, Chinese, Italian and Greek backgrounds. Patient Educ Couns 2006;64(1-3):360-8.

30. Elzen H, Slaets JP, Snijders TA, Steverink N. Evaluation of the chronic disease self-management program (CDSMP) among chronically ill older people in the Netherlands. Soc Sci Med 2007;64(9):1832-41. 
31. Goeppinger J, Armstrong B, Schwartz T, Ensley D, Brady TJ. Self-management education for persons with arthritis: Managing comorbidity and eliminating health disparities. Arthritis Rheum 2007;57(6):1081-8.

32. Kendall E, Catalano T, Kuipers P, Posner N, Buys N, Charker J. Recovery following stroke: the role of self-management education. Soc Sci Med 2007;64(3):735-46.

33. Kennedy A, Reeves D, Bower P, Lee V, Middleton E, Richardson G, et al. The effectiveness and cost effectiveness of a national lay-led self care support programme for patients with long-term conditions: a pragmatic randomised controlled trial. J Epidemiol Community Health 2007;61(3):254-61.

34. Ludman EJ, Simon GE, Grothaus LC, Luce C, Markley DK, Schaefer J. A pilot study of telephone care management and structured disease self-management groups for chronic depression. Psychiatr Serv 2007;58(8):1065-72.

35. Siu AM, Chan CC, Poon PK, Chui DY, Chan SC. Evaluation of the chronic disease self-management program in a Chinese population. Patient Educ Couns 2007;65(1):42-50.

36. Elzen H, Slaets JP, Snijders TA, Steverink N. The effect of a self-management intervention on health care utilization in a sample of chronically ill older patients in the Netherlands. J Eval Clin Pract 2008;14(1):159-61.

37. McGillion MH, Watt-Watson J, Stevens B, Lefort SM, Coyte P, Graham A. Randomized controlled trial of a psychoeducation program for the selfmanagement of chronic cardiac pain. J Pain Symptom Manage 2008;36(2):12640.

38. Richardson G, Kennedy A, Reeves D, Bower P, Lee V, Middleton E, et al. Cost effectiveness of the Expert Patients Programme (EPP) for patients with chronic conditions. J Epidemiol Community Health 2008;62(4):361-7.

39. Barlow JH, Turner AP, Gilchrist M. A randomised controlled trial of lay-led selfmanagement for Myocardial Infarction patients who have completed cardiac rehabilitation. Eur J Cardiovasc Nurs 2009.

40. Gitlin LN, Chernett NL, Harris LF, Palmer D, Hopkins P, Dennis MP. Harvest health: translation of the chronic disease self-management program for older African Americans in a senior setting. Gerontologist 2008;48(5):698-705.

41. Jaarsma T, Haaijer-Ruskamp FM, Sturm H, Van Veldhuisen DJ. Management of heart failure in The Netherlands. Eur J Heart Fail 2005;7(3):371-5.

42. Shively M, Kodiath M, Smith TL, Kelly A, Bone P, Fetterly L, et al. Effect of behavioral management on quality of life in mild heart failure: a randomized controlled trial. Patient Educ Couns 2005;58(1):27-34.

43. Hobbs FD, Kenkre JE, Roalfe AK, Davis RC, Hare R, Davies MK. Impact of heart failure and left ventricular systolic dysfunction on quality of life: a cross-sectional study comparing common chronic cardiac and medical disorders and a representative adult population. Eur Heart J 2002;23(23):1867-76.

44. Stewart AL, Greenfield S, Hays RD, Wells K, Rogers WH, Berry SD, et al. Functional status and well-being of patients with chronic conditions. Results from the Medical Outcomes Study. JAMA 1989;262(7):907-13.

45. Stewart S, Maclntyre K, Hole DJ, Capewell S, McMurray JJ. More 'malignant' than cancer? Five-year survival following a first admission for heart failure. Eur $J$ Heart Fail $2001 ; 3(3): 315-22$. 
46. Lee CS, Tkacs NC, Riegel B. The influence of heart failure self-care on health outcomes: hypothetical cardioprotective mechanisms. J Cardiovasc Nurs 2009;24(3):179-87; quiz 188-9.

47. Griffiths C, Foster G, Ramsay J, Eldridge S, Taylor S. How effective are expert patient (lay led) education programmes for chronic disease? BMJ 2007;334(7606):1254-6.

48. Sullivan MD, LaCroix AZ, Russo J, Katon WJ. Self-efficacy and self-reported functional status in coronary heart disease: a six-month prospective study. Psychosom Med 1998;60(4):473-8. 



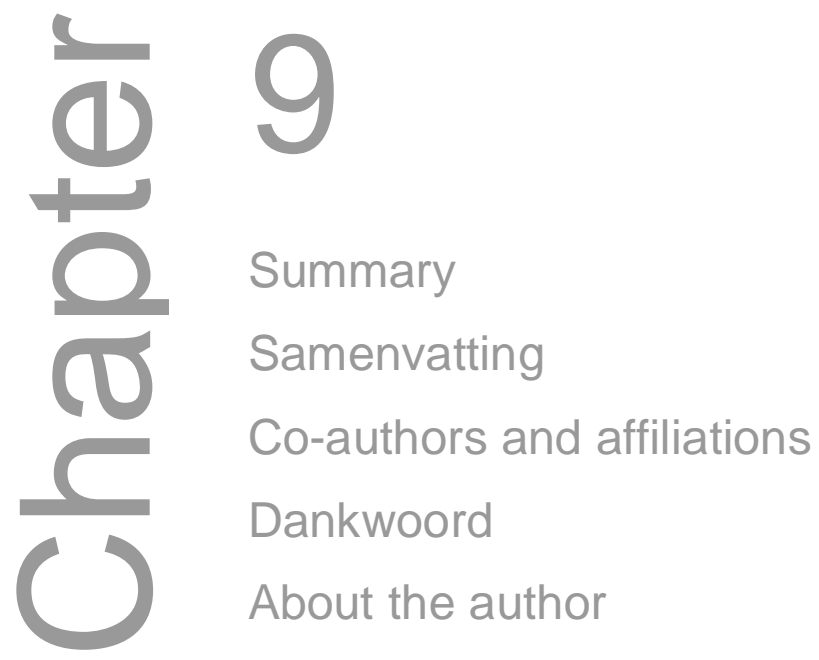




\section{Summary}

Managing congestive heart failure (CHF) is a societal and an individual challenge. A steady increase in the number of CHF patients and hospital admissions for $\mathrm{CHF}$ is predicted because of the ageing population, success in prolonging survival in patients suffering coronary events, and success in postponing coronary events by effective prevention strategies. For the individual patient accepting having a progressive disorder with a poor prognosis threatening daily functioning and quality of life is challenging. Other challenges for the patients are adhering to a comprehensive treatment regimen with multiple medications, dietary sodium and fluid restrictions, adopting a healthier lifestyle, recognizing symptoms of $\mathrm{CHF}$ deterioration and monitoring weight.

Patients' own abilities to manage their chronic condition in daily life may prevent CHF exacerbations and hospital readmissions. In the past ten years, much research has been conducted to improve self-management among CHF patients by providing education, for example, on lifestyle changes and selfmonitoring. Most interventions, however, have paid insufficient attention to the psychosocial aspects of CHF self-management. This is undesirable as besides physiologic factors, psychosocial factors such as depression and lack of social support are predictors for hospital readmissions. Therefore, we performed a randomized controlled trial to assess the effectiveness and feasibility of the Chronic Disease Self-Management Programme (CDSMP) among CHF patients in The Netherlands. The CDSMP elaborates on the definition that self-management deals with the medical, social and emotional consequences of being chronically ill and teaches patients to take responsibility for managing their chronic health problems on all three levels. The two objectives of the research presented in this thesis were: (1) to assess the effects of the CDSMP on psychosocial attributes, health behaviour, quality of life and healthcare utilization among CHF patients; and (2) to assess the feasibility of the CDSMP in terms of (a) the extent to which the intervention was performed according to protocol; (b) patients' adherence to the intervention; and (c) the opinions of the patients and leaders about the intervention.

Chapter 2 provides the results of a small-scale pilot study exploring the feasibility and possible benefits of the CDSMP among a group of ten older male patients with an implantable cardioverter defibrillator. The six weekly sessions were led by different pairs of trained cardiac nurse specialists and CHF patients. The programme was conducted largely according to protocol and both patients and leaders had favourable opinions about the programme. 
Improvements were identified for self-efficacy expectancies, symptoms of anxiety and on four subscales of quality of life.

Chapter 3 comprises the design of a two-group randomized controlled trial to assess the effectiveness and feasibility of the CDSMP among a large group of CHF outpatients in The Netherlands. Eligible CHF patients with a slight to marked limitation of physical activity (New York Heart Association (NYHA) Classes II and III) were recruited from six hospitals and were randomized to the intervention or control group. Patients in the intervention group received a six week self-management group programme led by a trained team comprising a cardiac nurse specialist and a CHF patient. Control patients received the usual care from regular checkups at the outpatient clinic. The outcomes of the effect evaluation were: psychosocial attributes (self-efficacy expectancies, perceived control, and cognitive symptom management), health behaviour (smoking and drinking behaviour, body mass, physical activity, and self-care behaviour), quality of life (health-related quality of life, perceived autonomy, and symptoms of anxiety and feelings of depression) and healthcare utilization (e.g. number of contacts with a cardiologist and number of hospitalizations). Data were collected before randomization, directly after the end of the programme and at six and twelve months after the start of the intervention. Feasibility data were collected directly after the end of the programme and at one year of follow-up.

Chapter 4 presents the effects of the CDSMP on psychosocial attributes, selfcare behaviour and quality of life. In total, $317 \mathrm{CHF}$ patients were randomly allocated to the self-management group programme $(n=186)$ or to usual care ( $n=131$ ). In total, $84 \%$ of the 317 patients completed the trial and $75 \%$ of the 186 patients in the intervention group attended at least four programme sessions. At baseline, both groups were comparable in terms of background characteristics and outcome measures, except for cardiac-specific quality of life on which intervention group patients scored significantly lower compared with control group patients. Outcomes of the statistical analyses were adjusted for baseline differences on cardiac-specific quality of life. Directly after the end of the programme significant favourable effects of the intervention were found for cognitive symptom management, self-care behaviour and cardiac-specific quality of life (after adjustment of baseline differences). No long-term effects of the CDSMP were found. In addition, the programme did not affect self-efficacy expectancies, perceived control, perceived autonomy, symptoms of anxiety and feelings of depression. 
In Chapter 5, the effects of the CDSMP on health behaviour and healthcare utilization are described. At baseline, both groups were comparable on all variables. Regarding health behaviour, directly after the end of the programme favourable effects were found for 'other physical activity' in terms of walking for exercise and physical activities such as aerobic, stretching and strengths exercises, sports and gardening. The effects of the programme on 'other physical activities' extended to six months of follow-up after the start of the programme. The programme did not affect smoking behaviour, drinking behaviour, body mass and healthcare utilization.

Chapter 6 presents the results of additional exploratory subgroup analyses assessing which patients benefited most from attending the CDSMP with respect to cardiac-specific quality of life. Cardiac-specific quality of life was measured at baseline, directly after the end of the programme and six and twelve months after the start of the intervention. Subgroup effects were assessed for disease-related (time interval from CHF diagnosis, NYHA classification, and active comorbid diseases), cognitive (cognitive status), and socio-demographic (sex, age, educational level, and living arrangements) patient characteristics as measured at baseline. If necessary, patient characteristics were dichotomised based on the median score of the total sample of 317 patients. The analyses showed that in the short term patients with better cognitive status benefited more from the CDSMP than their poorer functioning counterparts. However, no long-term difference in cardiac-specific quality of life was found between the two subgroups. Patients who attended at most lower secondary (vocational) education benefited more from the CDSMP than their higher educated counterparts during total follow-up with respect to cardiac-specific quality of life. No other subgroup effects were found. Future research is necessary to confirm the influence of cognitive status and educational level on the programme's effectiveness.

Chapter 7 presents the feasibility study that was performed among 186 intervention group patients and 18 trained leaders (i.e. nine cardiac nurse specialists and nine CHF patients). The main outcomes were performance according to protocol, patients' adherence and patients' and leaders' opinions about the programme. The feasibility study showed that $80 \%$ of the programme sessions were carried out according to protocol. In total, $75 \%$ of the patients attended at least four of the six programme sessions. Most patients made weekly action plans which they accomplished on a weekly basis. Both patients and leaders had favourable opinions about the programme. After attending the programme, at least $62 \%$ of the patients 
perceived themselves able to make behavioural changes, however, in the long term these numbers decreased. Both patients and leaders recommended taking additional time to discuss specific topics such as 'advance directives for health care'. Furthermore, they recommended to take differences in patient characteristics into account. Being female and having a lower NYHA classification (indicating better functioning) predicted good attendance to the programme.

Finally, in Chapter 8 the main findings of the research are discussed. Some methodological considerations are addressed and implications for practice and future research are provided. The main conclusion of the research presented in this thesis is that the CDSMP was considered feasible and showed favourable effects on several patient outcomes in the short term. However, most of these effects diminished in the longer term. Therefore, instead of implementing the CDSMP in CHF regular care, the programme must be adapted to improve its long-term effectiveness among CHF patients in The Netherlands. 


\section{Samenvatting}

De zorg voor patiënten met hartfalen blijft een grote uitdaging. Hartfalen wordt de epidemie van de toekomst genoemd, die nog grotere proporties zal aannemen door de toenemende vergrijzing van de samenleving en verbeterde behandeling van levensbedreigende cardiale aandoeningen. Ook voor de patiënt kan het omgaan met de ziekte een grote uitdaging betekenen. De patiënt moet leren leven met een progressieve ziekte die gepaard gaat met een slechte prognose. Bovendien worden hartfalenpatiënten ernstig beperkt in hun dagelijks functioneren wat zich vertaalt in een verminderde kwaliteit van leven. Daarnaast worden patiënten met hartfalen geacht hun leefstijl aanzienlijk aan te passen door hun medicatie volgens voorschrift in te nemen, zich te houden aan eventuele zout- en vochtbeperkingen, gezonder te leven, de symptomen van verslechtering van de ziekte te herkennen en regelmatig het lichaamsgewicht te controleren. Patiënten die niet voldoende in staat zijn deze aanpassingen in hun dagelijks leven te realiseren, kunnen in een neerwaartse spiraal terecht komen waarin verslechtering van de functionele status van de patiënt kan leiden tot een grotere zorgvraag en een verdere achteruitgang van de conditie van de patiënt.

Zelfmanagement lijkt een belangrijke rol te spelen bij hartfalenpatiënten in het voorkomen van verslechtering van het beloop van de ziekte en heropnames in het ziekenhuis. Het afgelopen decennium is veel onderzoek verricht naar het versterken van zelfmanagement van patiënten door middel van patiëntenvoorlichting. Patiëntenvoorlichting bij hartfalen richt zich onder meer op aanpassingen in de leefstijl en het herkennen van symptomen van verslechtering van de ziekte. Echter, in het merendeel van deze zelfmanagementprogramma's wordt slechts beperkt aandacht besteed aan de psychosociale aspecten van het omgaan met de aandoening. Dit is een onwenselijke situatie, aangezien psychosociale factoren, zoals depressie en het gebrek aan sociale steun als even belangrijke risicofactoren voor heropnames in het ziekenhuis worden beschouwd als fysieke factoren. Om deze reden is een gerandomiseerde experimentele studie uitgevoerd naar de effectiviteit en toepasbaarheid van het Chronic Disease Self-Management Programme (CDSMP) bij hartfalenpatiënten in de Nederlandse situatie. Uitgangspunt van de CDSMP is dat zelfmanagement zich richt op zowel medische, sociale als emotionele consequenties van het leven met een chronische aandoening. In dit programma leren de deelnemers om zelf verantwoordelijkheid te nemen voor het hanteren van de ziekte in het dagelijkse leven op de drie genoemde domeinen. De twee onderzoeksvragen zijn: (1) wat is de effectiviteit van de CDSMP op psychosociale attributies, gezondheidsgedrag, kwaliteit van leven en zorggebruik bij hartfalenpatiënten; 
en (2) in hoeverre is de CDSMP toepasbaar bij hartfalenpatiënten voor wat betreft (a) de mate waarin het programma wordt uitgevoerd volgens protocol; (b) de mate waarin patiënten het programma doorlopen zoals gepland ('adherence'); en (c) de oordelen van deelnemers en cursusleiders over het programma.

In Hoofdstuk 2 worden de resultaten beschreven van een pilotstudie naar de toepasbaarheid en effectiviteit van de Nederlandse versie van de CDSMP bij een groep van tien oudere hartpatiënten met een inwendige cardiale defibrillator. Het zelfmanagementprogramma bestond uit zes wekelijkse groepsbijeenkomsten van circa tweeënhalf uur en werd geleid door koppels van getrainde cursusleiders in steeds wisselende samenstelling, elk bestaande uit een gespecialiseerde verpleegkundige en een hartfalenpatiënt. Het programma werd grotendeels volgens protocol uitgevoerd en zowel de deelnemers als de cursusleiders hadden een positief oordeel over het programma. Tevens leek het programma een positieve invloed te hebben op ervaren competentie, angstgevoelens en enkele aspecten van kwaliteit van leven bij de deelnemers.

In Hoofdstuk 3 wordt de opzet beschreven van een gerandomiseerde experimentele studie naar de effectiviteit en toepasbaarheid van de CDSMP bij poliklinisch behandelde hartfalenpatiënten in Nederland. Vanuit zes verschillende ziekenhuizen werden poliklinisch behandelde hartfalenpatiënten met een matige tot aanzienlijke beperking in fysiek functioneren (New York Heart Association (NYHA) klasse II tot III) uitgenodigd om deel te nemen aan het onderzoek. Direct na de voormeting werden de patiënten aselect toegewezen aan de interventie- of controlegroep. Patiënten die werden toegewezen aan de interventiegroep werden uitgenodigd om deel te nemen aan het zes wekelijkse CDSMP programma gericht op zelfmanagement dat werd geleid door een getraind koppel van een gespecialiseerde verpleegkundige en een hartfalenpatiënt. Patiënten die werden toegewezen aan de controlegroep ontvingen enkel de standaardzorg bestaande uit periodieke controleafspraken op de polikliniek cardiologie. Als uitkomstmaten werden meegenomen in het onderzoek: psychosociale attributies (zoals ervaren competentie, ervaren controle en cognitieve symptoommanagement), gezondheidsgedrag (zoals rook- en drinkgedrag, lichaamsgewicht, mate van bewegen en zelfzorggedrag), kwaliteit van leven (zoals algemene en ziektespecifieke kwaliteit van leven, ervaren autonomie en gevoelens van angst en depressie) en zorggebruik (zoals aantal contacten met een cardioloog en het aantal ziekenhuisopnames). Gegevens over de effectiviteit 
van het programma werden op vier momenten verzameld: voor de start van het programma, direct na afloop van het programma en zes en twaalf maanden na start van de zelfmanagementcursus. Gegevens over de toepasbaarheid van het programma werden verzameld direct na afloop van het programma en na één jaar.

In Hoofdstuk 4 wordt het effect van de CDSMP op psychosociale attributies, zelfzorg en kwaliteit van leven beschreven. In totaal werden 317 hartfalenpatiënten geïncludeerd in de studie. Om praktische redenen werd tijdens de randomisatieprocedure besloten tot een ongelijke verdeling van patiënten over de interventie- en controlegroep: in totaal werden 186 patiënten toegewezen aan de interventiegroep en 131 patiënten aan de controlegroep. Van deze 317 patiënten doorliep 84\% het gehele onderzoek, 75\% van de 186 deelnemers aan de cursus nam tenminste aan vier groepsbijeenkomsten deel. Voor aanvang van het programma waren beide onderzoeksgroepen onderling vergelijkbaar wat betreft achtergrondkenmerken en uitkomstmaten, met uitzondering van ziektespecifieke kwaliteit van leven waarop patiënten in de interventiegroep significant slechter scoorden dan patiënten in de controlegroep. Voor dit verschil werd gecorrigeerd in de statistische analyses. Direct na afloop van het programma werden significante verbeteringen gevonden bij deelnemers wat betreft cognitief symptoommanagement, zelfzorg en ziektespecifieke kwaliteit van leven (na correctie voor uitgangsverschillen). Deze effecten verdwenen echter op de langere termijn. Bovendien bleek het programma geen invloed te hebben op ervaren competentie, ervaren controle en gevoelens van angst en depressie.

In Hoofdstuk 5 worden de effecten van de CDSMP op gezondheidsgedrag en zorggebruik beschreven. Voor aanvang van het programma waren beide onderzoeksgroepen onderling vergelijkbaar op deze uitkomstmaten. Direct na afloop van het programma werden significante positieve effecten gevonden voor de mate van bewegen, namelijk voor wandelen en 'andere fysieke activiteiten' zoals aerobic, rek- en strekoefeningen, het doen aan sport en tuinieren. Het effect van het programma op 'andere fysieke activiteiten' bleef tot zes maanden na start van de zelfmanagementcursus bestaan. Het programma had geen significante invloed op rook- en drinkgedrag, lichaamsgewicht en zorggebruik.

In Hoofdstuk 6 wordt beschreven welke patiëntengroepen met name baat hebben bij de CDSMP op het gebied van ziektespecifieke kwaliteit van leven. Deze uitkomstvariabele werd gemeten voor start van het programma, direct 
na afloop van het programma en zes en twaalf maanden na start van de zelfmanagementcursus. Daarnaast werden ziektegerelateerde (duur van de ziekte, NYHA classificatie en comorbide aandoeningen), cognitieve (cognitief functioneren) en sociodemografische (geslacht, leeftijd, opleidingsniveau en leef- en werksituatie) patiëntengegevens verzameld voor aanvang van het programma. Indien nodig werden variabelen gedichotomiseerd op basis van de mediaan van de totale onderzoekspopulatie van 317 patiënten. De analyses wezen uit dat op de korte termijn deelnemers met beter cognitief functioneren een betere ziektespecifieke kwaliteit van leven hadden in vergelijking met deelnemers die cognitief minder goed functioneerden. Echter, op lange termijn werden deze verschillen tussen beide subgroepen niet meer gevonden. Daarnaast bleken deelnemers met een lager opleidingsniveau (ten hoogste middelbaar onderwijs of lager beroepsonderwijs) over de gehele onderzoeksperiode meer baat te hebben bij de CDSMP wat betreft ziektespecifieke kwaliteit van leven, dan hoger opgeleide deelnemers. Er werden geen andere subgroepeffecten gevonden. Aanvullend onderzoek is nodig om de mogelijke invloed van cognitief functioneren en opleidingsniveau op de effectiviteit van het programma te kunnen bevestigen.

Hoofdstuk 7 beschrijft de resultaten van een procesevaluatie die werd uitgevoerd bij 186 patiënten in de interventiegroep en 18 getrainde cursusleiders (negen gespecialiseerde verpleegkundigen en negen hartfalenpatiënten). De aspecten die werden onderzocht waren: de mate waarin het programma werd uitgevoerd volgens protocol, de mate waarin patiënten het programma hebben doorlopen zoals gepland ('adherence') en de oordelen van deelnemers en cursusleiders over het programma. De procesevaluatie liet zien dat $80 \%$ van de programmabijeenkomsten volgens protocol verliep. Van de deelnemers woonde $75 \%$ tenminste vier van de zes groepsbijeenkomsten bij. Het merendeel van de deelnemers maakte wekelijks een actieplan dat zij ook met succes uitvoerden. Zowel de deelnemers als de cursusleiders hadden een positief oordeel over het programma. Na afloop van het programma achtte tenminste $62 \%$ van de deelnemers zich in staat om gedragsverandering te bewerkstelligen. Echter, op de lange termijn daalde dit percentage aanzienlijk. Zowel de deelnemers als de cursusleiders deden de aanbeveling om binnen het programma meer tijd vrij te maken voor het intensiever behandelen van specifieke onderdelen, zoals het onderdeel 'wilsverklaring'. Bovendien werd aanbevolen om meer rekening te houden met de grote onderlinge verschillen tussen deelnemers in de cursusgroepen. Vrouwen en deelnemers met een lagere NYHA-classificatie (een indicator voor beter fysiek functioneren) bleken een groter deel van de cursus- 
bijeenkomsten bijgewoond te hebben dan mannen en deelnemers met een hogere NYHA-classificatie.

Tenslotte worden in Hoofdstuk 8 conclusies van het onderzoek geformuleerd en worden de belangrijkste bevindingen bediscussieerd, waarbij de resultaten ook worden vergeleken met bevindingen uit ander onderzoek. Verder worden enkele methodologische aspecten van het onderzoek besproken en worden aanbevelingen voor de praktijk en voor verder onderzoek gedaan. Belangrijkste conclusie van het onderzoek beschreven in dit proefschrift is dat de CDSMP toepasbaar is bij hartfalenpatiënten. Bovendien laat het programma enkele korte termijn effecten zien. Deze effecten blijven echter op de lange termijn niet behouden. Op basis van deze resultaten wordt dan ook niet aanbevolen om de CDSMP in deze vorm te implementeren in de reguliere zorg voor hartfalenpatiënten in Nederland. Om lange termijn effecten bij hartfalenpatiënten in Nederland te kunnen bewerkstelligen is het wellicht nodig het programma op een aantal onderdelen aan te passen. 


\section{Co-authors and affiliations}

$\mathrm{T}$ (Ton) Ambergen, PhD

Department of Methodology and Statistics, Maastricht University/CAPHRI

School for Public Health and Primary Care, Maastricht, The Netherlands

BK (Barbara) Dijkman-Domanska, MD, PhD

Department of Cardiology, University Medical Centre Utrecht, Utrecht, The Netherlands

JThM (Jacques) van Eijk, PhD

Department of Social Medicine, Maastricht University/CAPHRI School for Public Health and Primary Care, Maastricht, The Netherlands

APM (Ton) Gorgels, MD, PhD

Department of Cardiology, University Hospital Maastricht, Maastricht, The Netherlands

JCM (Jolanda) van Haastregt, PhD

Department of Health Care and Nursing Science, Maastricht University/

CAPHRI School for Public Health and Primary Care, Maastricht,

The Netherlands

EFM (Liesbeth) van Hoef

Department of Integrated Care, University Hospital Maastricht, Maastricht, The Netherlands

JJJ (Josiane) Janssen-Boyne, RN, MA

Department of Cardiology, Department of Integrated Care, University Hospital Maastricht, Maastricht, The Netherlands

GIJM (Ruud) Kempen, PhD

Department of Health Care and Nursing Science, Maastricht University/

CAPHRI School for Public Health and Primary Care, Maastricht, The Netherlands

CLB (Cara) Lodewijks-van der Bolt, MD

Department of Cardiology, Atrium Medical Centre, Heerlen, The Netherlands 
ESTF (Esther) Smeulders

Department of Health Care and Nursing Science, Maastricht University/

CAPHRI School for Public Health and Primary Care, Maastricht,

The Netherlands

HEJH (Jelle) Stoffers, MD, PhD

Department of General Medicine, Maastricht University/CAPHRI School for Public Health and Primary Care, Maastricht, The Netherlands

NHKM (Nicole) Uszko-Lencer, MD

Department of Cardiology, University Hospital Maastricht, Maastricht, The Netherlands 


\section{Dankwoord}

Bij start van dit promotietraject leek het schrijven van een dankwoord zo ver weg. $\mathrm{Nu}$ is het moment daar om alle mensen te bedanken die mij tijdens mijn onderzoek hebben gesteund en geïnspireerd.

Allereerst wil ik mijn promotoren en copromotor Ruud Kempen, Jacques van Eijk en Jolanda van Haastregt bedanken. Ik ben blij dat jullie mij de kans hebben gegeven om dit promotieonderzoek te doen: gelukkig zagen jullie toen al hoe belangrijk dit onderzoek voor mij is. Ruud, als dagelijkse begeleider gaf je me voldoende ruimte om het 'mijn onderzoek' te laten worden. Mede door je vertrouwen en betrokkenheid kon ik mijn eigen koers varen en tevens een beroep op je doen indien nodig. Ik waardeer nog altijd je inspanningen tijdens de opstartfase, toen we samen diverse ziekenhuizen in Limburg en Brabant bezochten om cardiologen te enthousiastmeren voor de zelfmanagementcursus. Jacques, je hebt nooit onder stoelen of banken gestoken dat je vertrouwen in me hebt; tijdens het schrijven van revisies voor artikelen of vlak voor een presentatie op een internationaal congres. Dat heeft altijd veel voor mij betekend! Ik wil je ook bedanken voor de kritische vragen die je regelmatig stelde, waardoor ik artikelen nog scherper kon neerzetten. Jolanda, je was een kei in het becommentariëren van stukken, hoewel ik in het begin wel moest wennen aan je pragmatische aanpak als je hele alinea's wilde schrappen, haha. Heel bijzonder vond ik de gesprekken die we regelmatig met elkaar voerden over het doen van onderzoek en, vaker nog, over alle andere dingen die het leven zo waardevol en interessant maken! Tenslotte wil ik ook Liesbeth van Hoef op deze plaats bedanken. Liesbeth, als onderzoeksassistente op dit project hebben we de eerste twee en een half jaar als een waar 'dynamic duo' samengewerkt. Met al je onderzoekservaring ging je er in dit project helemaal voor en dat inspireerde mij enorm!

De leden van de beoordelingscommissie, voorzitter prof. dr. Bart van den Borne, dr. Tiny Jaarsma, dr. Petra Kuijpers, prof. dr. Joris Slaets en prof. dr. Rianne de Wit, wil ik bedanken voor het beoordelen van dit proefschrift. Dank ook aan de leden van de projectgroep en/of co-auteurs van de diverse artikelen: Josiane Janssen, Jelle Stoffers, Nicole Lencer, Ton Gorgels, Cara Lodewijks, Barbara Dijkman en Ton Ambergen. Josiane, ondanks dat je zo druk bent met je werk in het ziekenhuis en je eigen promotietraject, ben je altijd actief betrokken geweest in de projectgroep. Ik bewonder je doorzettingsvermogen en wens je heel veel succes met al je inspanningen op hartfalengebied. Jelle, bedankt voor je heldere blik tijdens discussies en bij het becommentariëren van diverse artikelen. Nicole, Ton, Cara en Barbara: jullie 'cardiale' bijdrage tijdens het onderzoek was zeer waardevol, bedankt hiervoor! Tenslotte wil ik Ton Ambergen bedanken voor zijn begeleiding op 
statistisch gebied; ik ben niet meer zo huiverig voor het doen van multilevelof subgroepanalyses.

Dit onderzoek had weinig kans van slagen gehad zonder twee groepen mensen: de deelnemers en de cursusleiders. Allereerst wil ik mijn waardering uitspreken voor de deelnemende patiënten van het academisch ziekenhuis Maastricht, het Atrium Medisch Centrum in Heerlen, het VieCuri Medisch Centrum in Venlo, het Laurentius Ziekenhuis in Roermond, het Maasland Ziekenhuis in Sittard en het Elkerliek Ziekenhuis in Helmond. Trouw verleende $u$ medewerking aan een intensieve dataverzameling en bezocht $u$ de bijeenkomsten van de zelfmanagementcursus. Ik vond het heel bijzonder om uw persoonlijke verhalen en ervaringen tijdens het onderzoek te mogen aanhoren of lezen in uw persoonlijke brieven. Ik hoop van harte dat het onderzoek ook voor $u$ van betekenis is geweest. Op deze plaats wil ik verder iedereen in de deelnemende ziekenhuizen bedanken die het mogelijk hebben gemaakt om het onderzoek uit te voeren; van de inclusie van patiënten tot het beschikbaar stellen van cursusruimtes.

Heel veel bewondering heb ik verder voor 'mijn modelpatiënten': Frans Biesmans, Jan Cuenen, Jan Mommers, Huub Mulders, Jacques Koonen, Toon Zijlstra, Huub Clignet, Ad van de Broek, Jan Verspaget en Jan-Willem Deetman. Naast het mede begeleiden van de cursusgroepen, gaven jullie het onderzoek 'een gezicht', wat mij bijzonder motiveerde. Jullie zijn stuk voor stuk kanjers, het gaat jullie goed! Ook wil ik op deze plaats de 'professionele cursusleiders' bedanken voor het begeleiden van de cursusgroepen: Marianne Beijer, Judith Jehle, Jacqueline Jans, Liliane Moors, Sandra Palmen, Theo Thuis, Vera Post, Sabrina Jongen, Pia Coppin en Marcel Peeters. Ik hoop dat de zelfmanagementcursus jullie allen nog altijd inspireert in jullie werk! Marianne en Judith, ik denk nog met veel plezier terug aan onze tijd op Stanford University bij Kate Lorig en aan de eerste training die we zelf gaven weer terug in Maastricht. Marianne, we raakte steeds beter op elkaar ingespeeld wat betreft het trainen van cursusleiders. Ik vond het een heel waardevolle ervaring.

Vervolgens wil ik de collega's van het MEMIC bedanken: Marlène Ronner en de enquêtrices wil ik bedanken voor hun meer dan flexibele inzet tijdens het uittesten en uitvoeren van de telefonische interviews. Ook Annemie Mordant, Anita Legtenberg en Jack Berben wil ik danken voor de prettige samenwerking. Tenslotte, Alfons Schroten, hartelijk dank voor het mede uitdenken, bouwen, testen en optimaliseren van onze eigen hartfalenrolodex! Verder wil ik op deze plaats Vonca Schaffers bedanken voor haar assistentie bij het compleet krijgen van de schriftelijke vragenlijsten. 
Mijn oud-collega's bij Medische Sociologie wil ik bedanken voor de fijne tijd. Marjoke, bedankt voor je secretariële ondersteuning en de prettige gesprekken. Je 'THEE!' blijft me altijd bij. Arnold, jouw ICT-ondersteuning ging tot voorbij de provinciale grens; bedankt voor je snelle acties in al die jaren! Beste junioren, bedankt voor de gezellige tijd op de afdeling en tijdens AIOsoepen of andere nevenactiviteiten. Ik heb me bij jullie als een vis in het water gevoeld! Bedankt dat ik ook altijd bij jullie terecht kon voor persoonlijk advies. Ook wil ik mijn collega's van Verpleging \& Verzorging bedanken voor hun hartelijke ontvangst in de vakgroep en hun interesse in mijn onderzoek. Tenslotte wil ik mijn nieuwe collega's in Brabant, evenals mijn collega's bij de Nederlandse Hartstichting, bedanken voor hun oprechte interesse voor mijn promotieonderzoek.

Mijn familie en vrienden wil ik hartelijk danken voor hun belangstelling in de afgelopen jaren. Ik ben blij dat ik jullie nu kan laten zien waar ik toch de afgelopen jaren zo druk mee ben geweest. Opa's en oma's: bedankt voor jullie flexibiliteit in het afgelopen jaar om op te passen als ik weer eens naar Maastricht moest of gewoon flink wilde doorwerken aan het proefschrift. Lieve Jolien en Marja, ik vind het heel bijzonder dat jullie mijn paranimfen willen zijn op deze speciale dag! Jolien, bedankt voor je hechte vriendschap en de lol die we samen hebben. Het voelt goed dat ik altijd op je kan bouwen! Marja, mede dankzij jouw enthousiasme bij de junioren, voelde de AIO-tijd als een warme deken; ik ben blij dat we nog altijd vriendinnen zijn. Nog een paar maandjes en dan ben jij eindelijk aan de beurt!

Lieve pap, lieve Judith, ik ben dankbaar voor jullie onvoorwaardelijke liefde en steun. Ik weet zeker dat jullie vandaag voor drie genieten! Lieve mam, waar je ook bent, je voelt altijd dichtbij! Ik wil jou en papa hartelijk bedanken voor de solide basis die jullie mij hebben gegeven. Ik weet dat jullie trots op me zijn!

Tenslotte, lieve Bas, je staat al een hele poos naast mijn zijde. Je bent mijn rots in de branding en ik heb al veel van je geleerd. Bedankt voor je niet aflatende vertrouwen in mijn kunnen en de rust die je brengt. En de laatste woorden zijn voor jou, m'n kleine meid... Lieve Tess, jij bent echt mijn allergrootste inspiratiebron! 


\section{About the author}

Esther Smeulders was born on April 17th, 1978 in Loon op Zand, The Netherlands. After completing her secondary education (Gymnasium) at the Dr. Mollercollege in Waalwijk in 1996, she studied Social Work (SPH) at the Hogeschool West-Brabant in Breda for which she obtained her Propaedeutics degree. In 1998 she started to study Health Sciences at Maastricht University. She completed her masters in Health Education and Promotion and in Work and Health in 2003. In December of that year, she began with her PhD project on self-management among patients with congestive heart failure, which she conducted at the Faculty of Health, Medicine and Life Sciences and CAPHRI School for Public Health and Primary Care. The project, as described in this thesis, included a longitudinal randomized controlled trial to assess the effectiveness and feasibility of an American cognitive-behavioural selfmanagement group programme among congestive heart failure outpatients in The Netherlands. Since October 2008, she trains healthcare practitioners at the Netherlands Heart Foundation in creating and leading support groups for congestive heart failure patients. In addition, since June 2009 she is working as a policy maker for "De ZorgAlliantie", an alliance of several large healthcare organizations in North-East Brabant, who are joined together to improve cure and care for older people in the region Oss-Uden-Veghel. 